HIFAN 1602

\title{
DARHT-II Injector Transients and the Ferrite Damper
}

\section{Richard Briggs, Will Waldron, Lou Reginato, Ken Chow, Tim Houck, Enrique Henestroza, Simon Yu, and Michael Kang}

Accelerator Fusion Research Division

Ernest Orlando Lawrence Berkeley National Laboratory

University of California

Berkeley, California 94720

\section{August 2006}

This work was supported by the Director, Office of Science, Office of Fusion Energy Sciences, of the U.S. Department of Energy under Contract No. DE-AC02-05CH11231. 


\section{DARHT-II Injector Transients and the Ferrite Damper}

Richard Briggs, Will Waldron, Lou Reginato, Ken Chow, Tim Houck, Enrique Henestroza, Simon Yu, and Michael Kang

August 4, 2006 


\section{Table of Contents}

\section{Introduction}

\section{Injector Diagnostics}

2.1 Stalk Current: Segmented Rogowski Coil Monitor

2.2 Source and MARX Dome Voltages: Capacitive Voltage Monitor

2.3 Column Current and MOV Current: Current Viewing Resistors (CVR)

2.4 Cathode Current Viewing Resistors (CVR)

2.5 Beam Position Monitor (BPM)

2.6 B-Dot Loops

2.7 Photo Multiplier Tube (PMT): Arc Detection

2.8 Gated Cameras: Arc Location

\section{Injector Transients}

3.1 Introduction

3.2 Injector waveforms

3.3 A Simple Lumped Circuit Model of the Injector

\subsubsection{Parameter Selection}

3.3.2 Excitation Level of the 7.8 $\mathrm{MHz}$ Oscillations

3.3.3 Damping Rate of the 7.8 MHz Oscillations without Beam

3.3.4 Voltage Rise Time and 7.8 MHz Oscillation Damping Rate with a $1.2 \mathrm{kA}$ Beam

3.4 Column Rise Time Transients

3.5 Crowbar Operation

\subsubsection{EM Mode Simulations}

3.5.2 Modifications in the Crowbar Circuitry

\section{Design of the Ferrite Damper and Small Scale Circuit Tests}

4.1 Introduction

4.2 Design Concepts

4.3 Small Scale Tests 
4.3.1 The "No Ferrite Damper" Baseline

4.3.2 Ferrite with a 9 mil Gap, No Beam Configuration

4.3.3 Ferrite with a 9 mil Gap in the "With Beam" Configuration

4.3.4 Ferrite with No Gap in the "With Beam" Configuration

4.4 Conclusions

\section{Ferrite Damper Mechanical Details and Installation}

5.1 General Assembly

5.1.1 Ferrite Tiles

5.1.2 Gaps Between Tiles (Gap Spacer and Wedge Pieces)

5.1.3 Back Spacers

5.1.4 Shelf System

5.1.5 Diagnostic Loops in Wedge Pieces

5.2 Oil Bubble Test

5.3 Installation

5.3.1 Work Platform

5.3.2 Mockup Marx Skirt

5.3.3 Tooling

\section{Ferrite Damper Performance}

6.1 Observed Damping by the Ferrite Insert

6.2 Analysis of a "No Beam" Shot (\#3064)

6.3 Analysis of a $\sim 1$ kA Beam Shot (\# 3054)

\section{Conclusion}

\section{References}




\section{Introduction}

This report summarizes the transient response of the DARHT-II Injector and the design of the ferrite damper. Initial commissioning of the injector revealed a rise time excited 7.8 $\mathrm{MHz}$ oscillation on the diode voltage and stalk current leading to a $7.8 \mathrm{MHz}$ modulation of the beam current, position, and energy. Commissioning also revealed that the use of the crowbar to decrease the voltage fall time excited a spectrum of radio frequency modes which caused concern that there might be significant transient RF electric field stresses imposed on the high voltage column insulators. Based on the experience of damping the induction cell RF modes with ferrite, the concept of a ferrite damper was developed to address the crowbar-excited oscillations as well as the risetime-excited 7.8 MHz oscillations. After the Project decided to discontinue the use of the crowbar, further development of the concept focused exclusively on damping the oscillations excited by the rise time. The design was completed and the ferrite damper was installed in the DARHT-II Injector in February 2006.

The organization of this report is as follows. The suite of injector diagnostics are described in Section 2. The data and modeling of the injector transients excited on the rise-time and also by the crowbar are discussed in Section 3; the objective is a concise summary of the present state of understanding. The design of the ferrite damper, and the small scale circuit simulations used to evaluate the ferrite material options and select the key design parameters like the cross sectional area and the optimum gap width, are presented in Section 4. The details of the mechanical design and the installation of the ferrite damper are covered in Section 5. A brief summary of the performance of the ferrite damper following its installation in the injector is presented in Section 6 . 


\section{Injector Diagnostics}

The column and diode diagnostics described in this Section are shown in Figure 2.1.

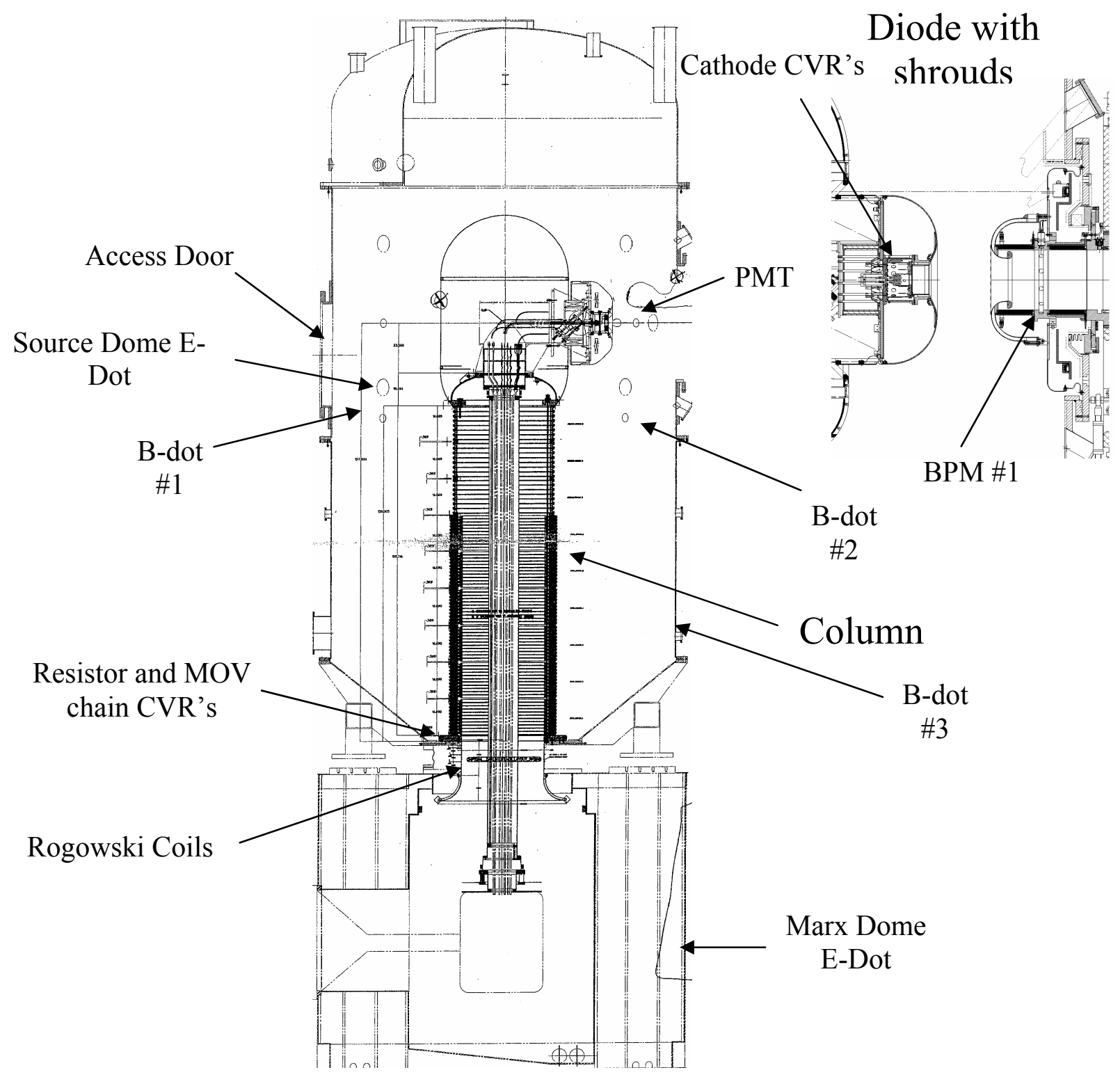

Figure 2.1 Schematic of injector showing approximate locations of diagnostics.

\subsection{Stalk Current: Segmented Rogowski Coil Monitor}

The monitor consists of four 5-turn coils positioned around the column. The radius of the coils is 0.5725 inches (to center of wire) giving a cross sectional area of $6.64 \mathrm{~cm}^{2}$. A schematic of one of the coils is shown in Figure 2.2. A measurement of the self 
inductance of one of the Rogowski loops was made at LBNL by Will Waldron and Lou Reginato. The loop looked like a linear inductor up to $15-20 \mathrm{MHz}$ (from a network analyzer test). The measured inductance was $\mathrm{L}=0.41 \mu \mathrm{H}$. This inductance is reasonable including the wire self inductance (about half the total).

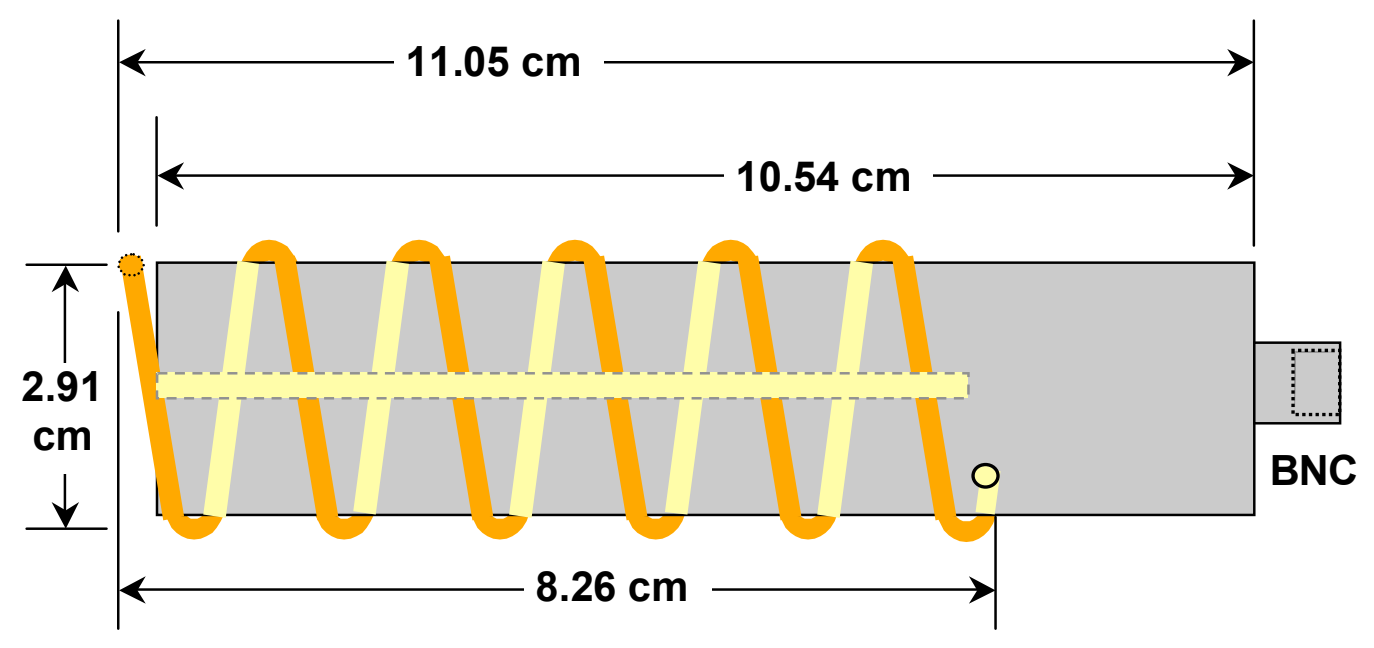

Figure 2.2 Schematic of one of the Rogowski coils used in the injector.

The coil is connected to a $50 \Omega$ coax cable giving a time response:

$$
\tau=\frac{L}{R}=8 \mathrm{~ns} .
$$

To compensate for the time response, or signal droop, when integrating the output signal:

$$
\int V_{\text {Rogows } s i} d t=\int V_{\text {out }} d t+\tau V_{\text {out }} .
$$

The coils are arranged around the stalk such that the azimuthal magnetic field produced by current flowing through the stalk is aligned with the axis of the coil. Neither an in situ nor "air line" calibration of the response of the coils to the stalk current has been performed. However, an estimate can be easily calculated:

$$
V_{\text {Rogowssi }}=N A \frac{\partial B_{\theta}}{\partial t}=N A \frac{\mu_{o}}{2 \pi r} \frac{\partial I}{\partial t} \text { and } I=\frac{2 \pi r}{N A \mu} \int V_{\text {Rogowsid }} d t,
$$

where I is the current flowing through the stalk, $\mathrm{r}$ is the radial distance from the stalk axis to the center of the coil, and $V_{\text {Rogowski }}$ is the induced voltage across the output of the coil.

This simple estimate does not include shielding of the magnetic field by metallic surfaces around the coil and will be low. During the "flat top" portion of the beam where displacement current can be ignored, the stalk current should equal beam current plus 
current flowing through the column. As discussed in Section 3, this cross check indicates that the simple estimate is about $10 \%$ low. Working with units of $\mathrm{kA}$ and $\mu \mathrm{sec}$, the best calibration for the Rogowski coils is:

$$
I=1.1 \times 10^{9} \int V_{\text {Rogowski }} d t
$$

In operation, the signals of the four coils are combined in a four-port summer producing an averaged output. It is possible to record the signals individually.

\subsection{Source and MARX Dome Voltages: Capacitive Voltage Monitor}

The voltages on the Source and MARX Domes are monitored with basic capacitive $\partial V / \partial t$ pickups. Thus,

$$
V_{\text {dome }}=\frac{1}{C_{p} Z_{o}} \int V_{\text {scope }} d t \text {, where }
$$

$C_{p}$ is the capacitance between the dome and the monitor, $Z_{o}$ is the impedance of the diagnostic line, $V_{\text {scope }}$ is the output voltage of the monitor. The monitors have been calibrated in situ using a voltage resistive divider on the MARX Dome and this calibration has been cross checked by measuring the beam energy.

\subsection{Column Current and MOV Current: Current Viewing Resistors (CVR)}

Multiple strings of resistors and MOV's join each adjacent pair of grading rings along the column (see Figure 2.3). For the bottom most pair of grading rings, each chain (4 resistors and $6 \mathrm{MOV}$ 's) is terminated to ground through a $125 \mathrm{~m} \Omega$ resistor. The voltage across the current viewing resistor $(\mathrm{CVR})$ is monitored and the total current flowing down the column can be determined.

$$
I_{\text {column }}=4 \times \frac{V_{\text {resitor }}}{0.125}+6 \times \frac{V_{M O V}}{0.125}, \text { where }
$$

$V_{\text {resistor }}$ and $V_{M O V}$ are the averaged voltage through a resistor chain CVR or a MOV chain CVR respectively. For normal conditions the MOV's will not transmit, so only the resistor portion of the current is important. Note also that the CVR's are only monitoring the current flow through the strings for the lowest pair of grading rings. There is no information concerning the strings on the other grading rings.

In operation, the signals of the four resistor chains are combined in a four-port summer and the six MOV's chains are combined in a six-port summer producing averaged outputs. It is possible to record any of the signals individually. 


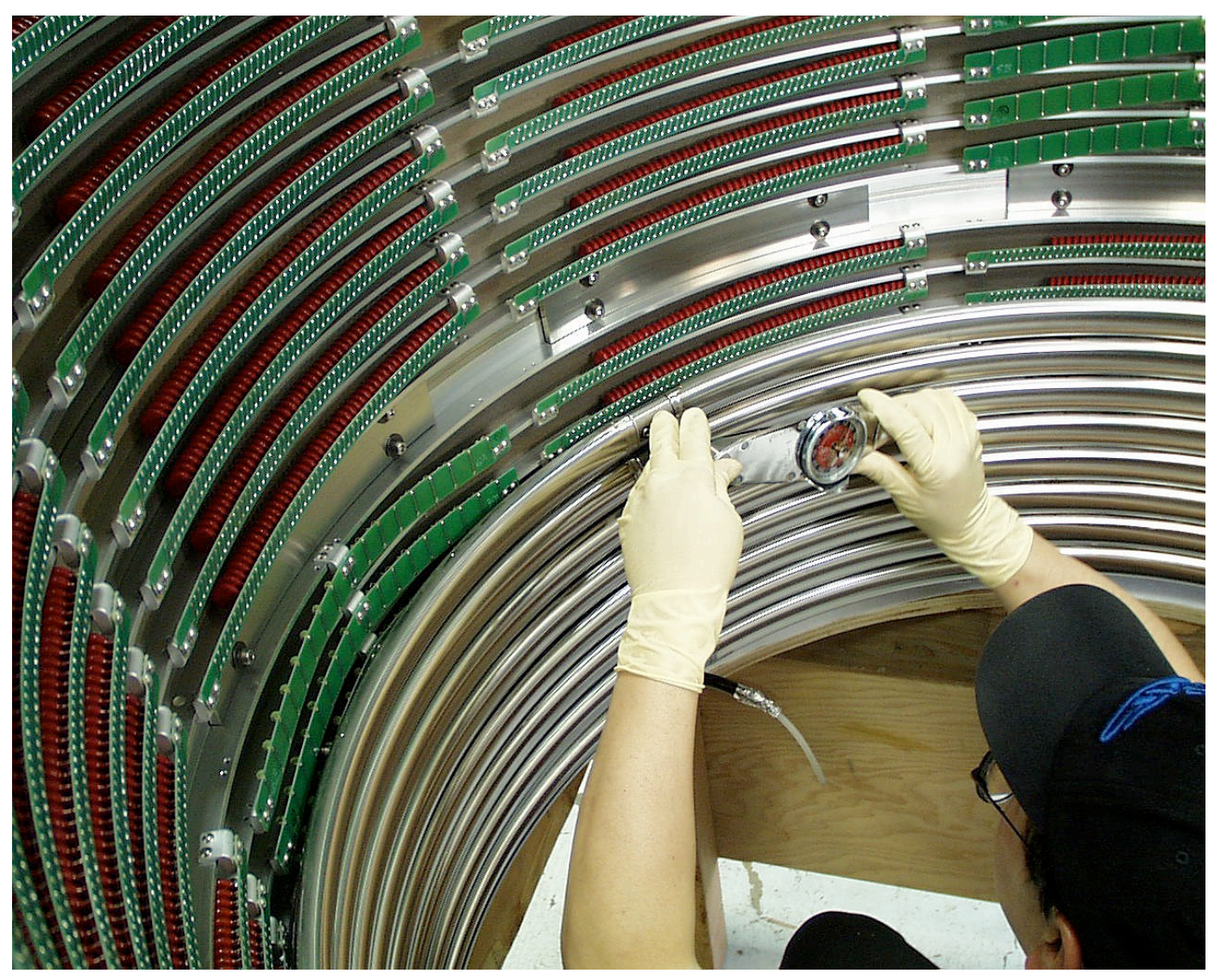

Figure 2.3 View of the inside of the column during assembly showing the resistor and MOV chains.

\subsection{Cathode Current Viewing Resistors (CVR)}

Twelve resistors are positioned azimuthally at 30-degree increments around the source between the source support tube flange (i.e. source) and the cathode support plate (i.e. cathode shroud). The source tube is electrically isolated from the cathode shroud except through current viewing resistors thus directing $I_{C V R}$ to flow from the cathode shroud through the CVR into the cathode source. This diagnostic allows a direct measurement of the emission from the source.

Each CVR resistor is made of a 2-mil foil of non-magnetic 304 stainless steel (s.s.) strip folded around a thin, 5-mil, DuPont Kapton ${ }^{\circledR}$ foil insulator (see Figure 2.4). The CVR voltage, $V_{C V R}$, is measured between two tabs cut in the s.s. foil.

The $V_{C V R}$ signal of each of the 12 CVR resistors is connected through a twisted pair (silvered stainless steel wires in a Teflon tube) to a BNC connector positioned under the cathode base plate. To attenuate any ground loop currents, the twisted pairs are passed through small ferrite rings. The $12 V_{C V R}$ signals are then carried on 12 vacuum, 50-ohm 
coaxial cables to allow easy access and connected in sets of three to four distribution boxes. Each set of three cables is from CVR resistors located in one quadrant around the source. Only four of the $V_{C V R}$ signals, one from each distribution box, are conveyed to Marx dome to be measured on four fast Tektronix digitizers due to space limitations. These signals represent the voltage across four CVR resistors 90 degrees apart selected at the distribution box.
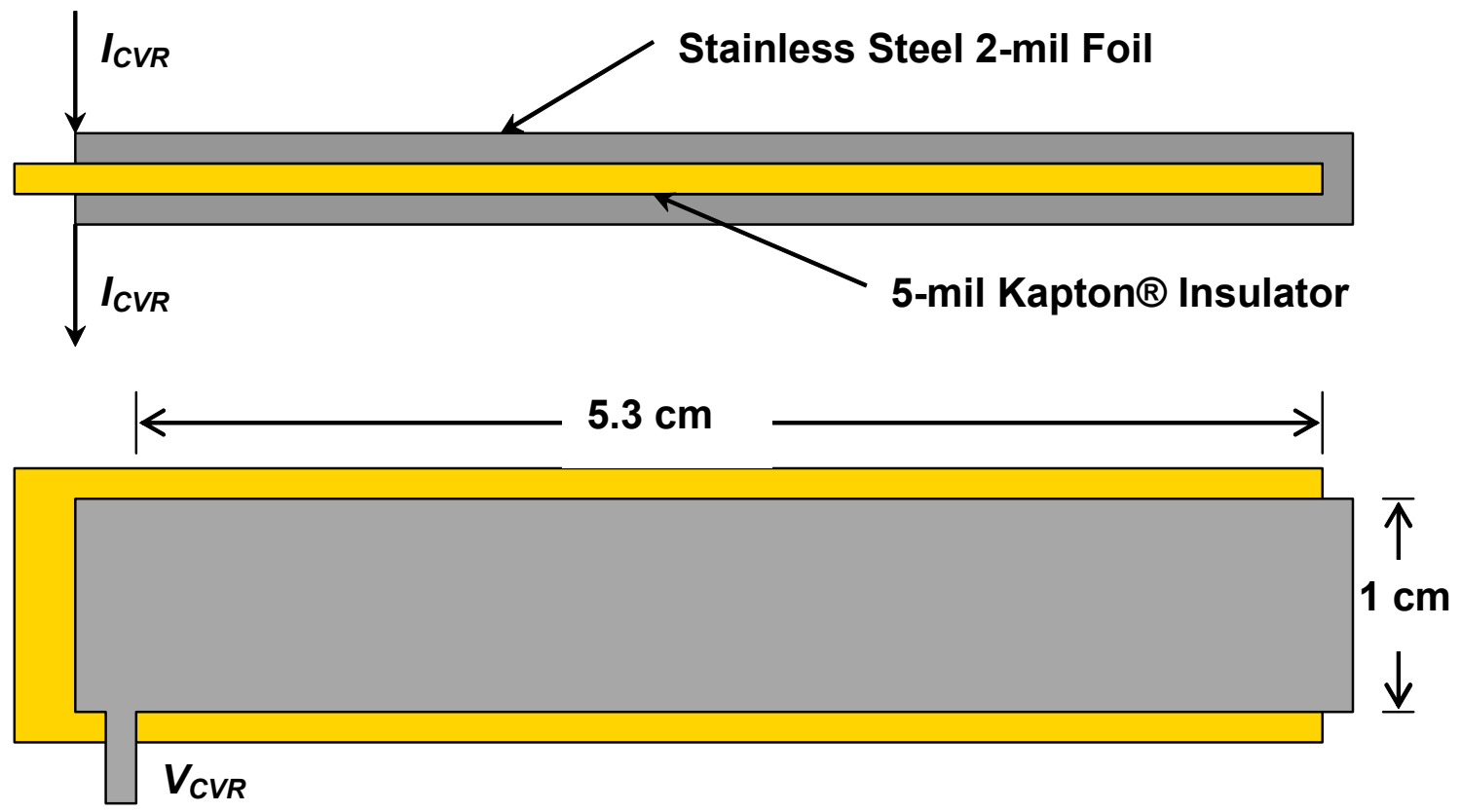

Figure 2.4 Schematic of a single current viewing resistor.

The calculated resistance per CVR is $141 \mathrm{~m} \Omega$. Measurements made by Shmuel Eylon at $\mathrm{LBNL}$ indicate that the resistance increases by about $6.5 \% / 100^{\circ} \mathrm{C}$ increase in temperature. Estimating the operational temperature of the CVR's as $250^{\circ} \mathrm{C}$ above room temperature (somewhat above $300^{\circ} \mathrm{C}$ on the cathode side and a steep gradient across the CVR to the water cooled side) the resistance would be $164 \mathrm{~m} \Omega$. Thus the calibration factor is $12 / 0.164 \mathrm{~A} / \mathrm{V}=73.2 \mathrm{~A} / \mathrm{V}$.

The CVR's worked correctly at the beginning of the Injector commissioning, but subsequently failed, possibly due to the higher than expected temperatures related to the initial heat shield configuration. The CVR's have not been checked since the new heat shields have been installed due to an incompatibility between the older digitizers in the MARX Dome and the new DARHT-II data acquisition system.

\subsection{Beam Position Monitor (BPM)}

The DARHT-II BPM is an azimuthal array of four B-dot detectors of a balanced design to provide protection from common mode signals, such as radiation driven Compton 
currents, direct electron impact, electrostatic pickup, and ground loops [1]. The balanced design b-dot loops have two output voltage signals, one equal to the rate of change of flux plus any common mode, the other equal to the negative of the change in flux plus any common mode. Thus, subtracting the two signals results in twice the rate of change of flux, and the common mode cancels.

Summing and integrating the outputs of the B-dots produces a signal proportional to the total beam current. Appropriate differencing and integration of the outputs produces signals proportional to the displacement of the beam centroid from the axis of the BPM. Assuming that all the current from the source is transported into the Anode tube, and no emission occurs from the cathode shroud, the first BPM provides a measurement of the emission from the cathode.

\subsection{B-Dot Loops}

In addition to the sophisticated and well-calibrated B-Dots installed in the BPM arrays, three large area B-Dots are installed in nominal 6" diameter ports around the vacuum vessel (Figure 2.5). The B-Dots have been used for relative measurements of RF magnetic fields and determining their frequency spectrum and mode pattern.

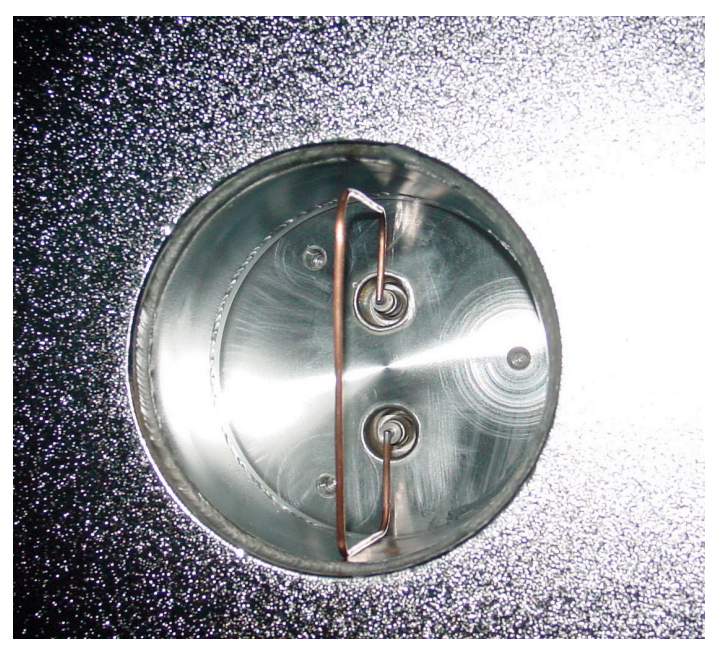

Figure 2.5 B-Dot loop. 


\subsection{Photo Multiplier Tube (PMT): Arc Detection}

A filtered photo multiplier tube is located at accelerator axis level on a port in the injector vacuum vessel with direct line of sight with the diode. The PMT is used to detect any light associated with a diode arc. However, due to the sensitivity of the PMT and the reflective interior of the vacuum vessel, any arc within the vacuum vessel including along the column will be detected. The PMT output signal provides temporal information on the arc, but no spatial location.

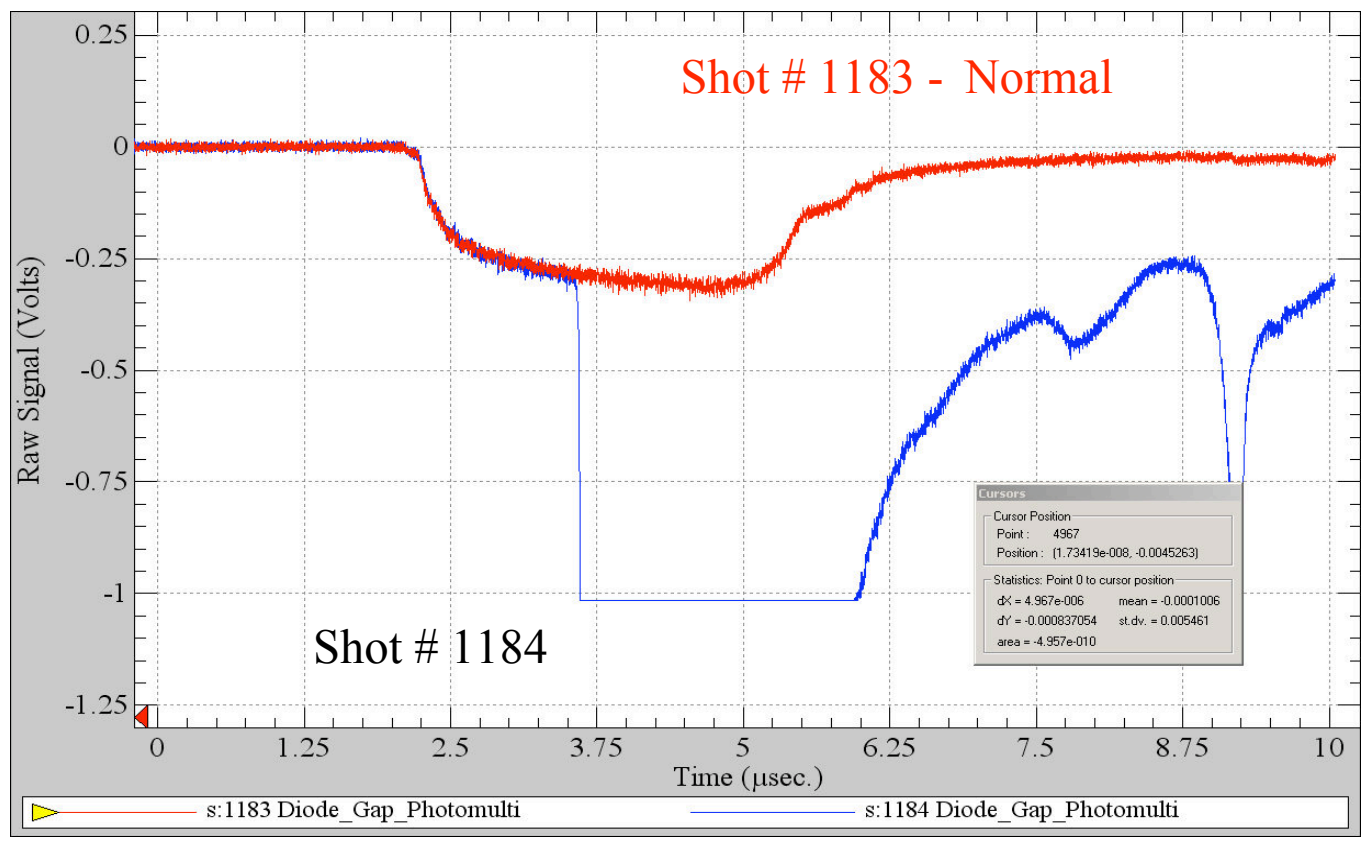

Figure 2.6 Output from the PMT.

A sample of the PMT output is shown in Figure 2.6. The red curve shows the signal for normal operation where a small signal is recorded during pulse time. The blue curve shows a signal associated with an abnormal event. For this event, light produced about midway through the pulse led to a large output signal that saturated the digitizer attached to the PMT.

\subsection{Gated Cameras: Arc Location}

Cameras are used to determine the location of arcs inside the vacuum vessel. Presently three cameras are mounted on ports along the vessel. One views the diode region and the other two view opposite sides of the column. No arcs have occurred since the cameras were installed. 


\section{Injector Transients}

\subsection{Introduction}

In this section, we present waveform and frequency spectrum data obtained with the principal injector diagnostics together with the results from various models, to elucidate the basic transient phenomenology. The objective is a concise summary of the present state of understanding.

The main features of the injector transients are dominated by the physical structure comprised of the Marx dome, stalk, column, and source dome. The Marx PFN itself does not appear to play an important role in the transients, based on the simple models presented in this section, so we will not discuss it in any detail here.

\subsection{Injector waveforms}

A typical set of the waveforms obtained with the key injector diagnostics are shown in Figures 3.1 and 3.2 (for shot \# 2264, data provided by Tim Houck). The Source dome voltage together with the Marx dome voltage are shown in Figure 3.1, while the stalk Rogowski, column current monitor at the base of the column, and the beam current at the exit of the diode (BPM01) are shown in Figure 3.2. (Note: at the time of this report a precise Rogowski calibration had not been established, so its amplitude was adjusted to equal the sum of the column current and the beam current in the flat top region. This was within 10\% of Tim Houck's calibration estimated from the loop area and location that was presented in Section 2.1. The Marx E-Dot calibration was also adjusted by $7.5 \%$ to fit the source dome amplitude.)

The principal features of the rise time transients observed with these diagnostics include the following:

- A strong oscillation around 7.8 $\mathrm{MHz}$ most clearly displayed in the Rogowski current, and also evident in the source dome voltage. This produces a modulation in the electron beam current (shown in Figure 3.2), energy, and position. This performance-degrading feature is one of the main motivations for installing the ferrite damper discussed in Section 4.

- A $10-90 \%$ rise time of the Marx and source dome voltages around $400 \mathrm{~ns}$ (efolding rise time around $190 \mathrm{~ns}$ ). This rise time is dominated by the capacitance of the domes and stalk. The initial "jump" in the stalk current and column current is in fact the capacitive current required to charge up these large structures. 


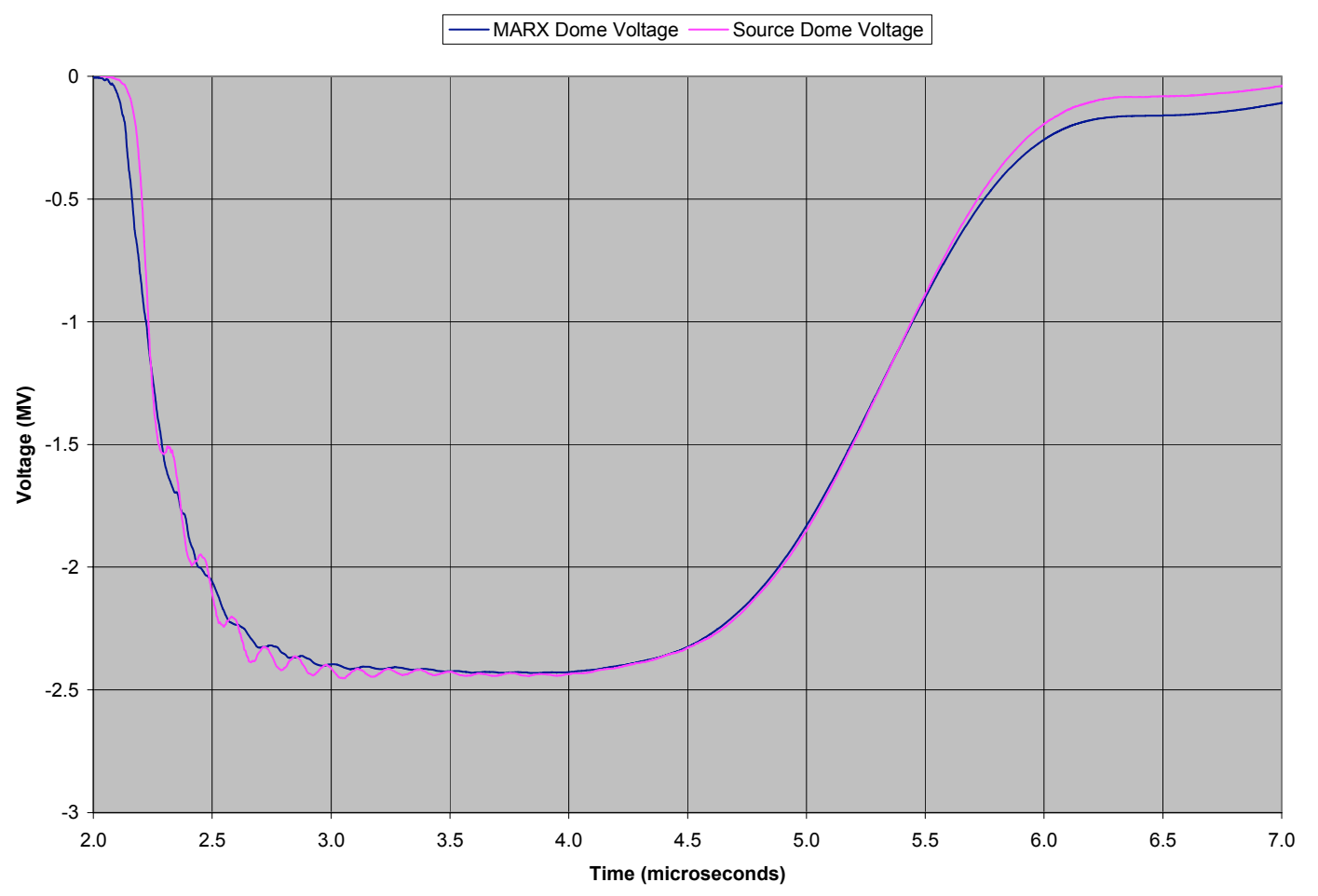

Figure 3.1 Source dome and Marx dome voltage waveforms.

Note that the column current asymptotes to a level around 450 amps after the rise time transients die out. This level is consistent with the steady column current expected with a net grading resistance of 5.3 Kohms, namely

\subsection{MeV/5.3 Kohms = 450 amps}

The time window of 2 to 7 microseconds shown in Figures 3.1 and 3.2 was chosen to display in detail the main pulse waveform. However it is also important to keep in mind that in operating without a crowbar, the long term ringing of the Marx PFN results in "secondary" lower energy beam pulses from the injector, as shown in Figure 3.3. 


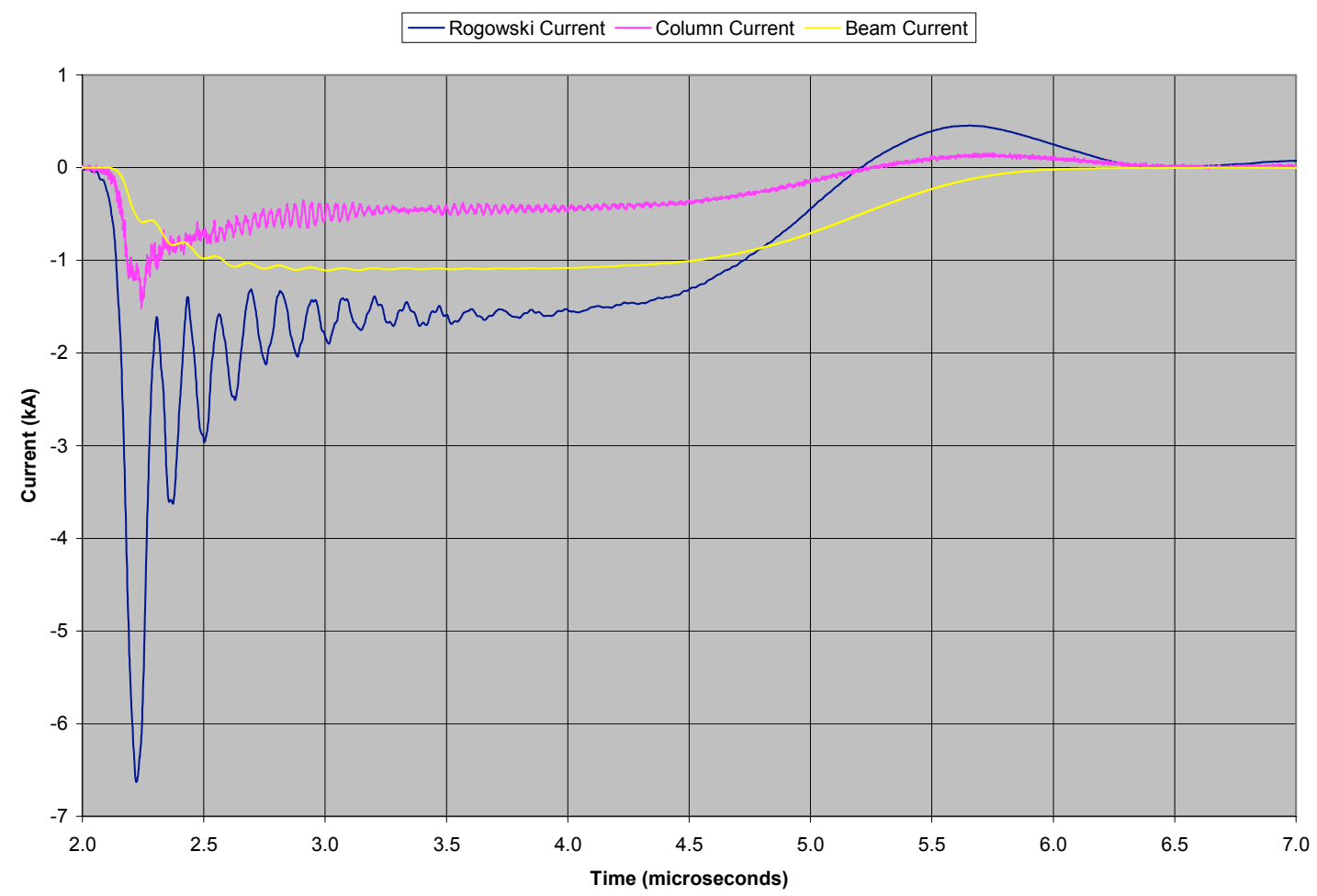

Figure 3.2 Stalk current, column current, and beam current at BPM01.

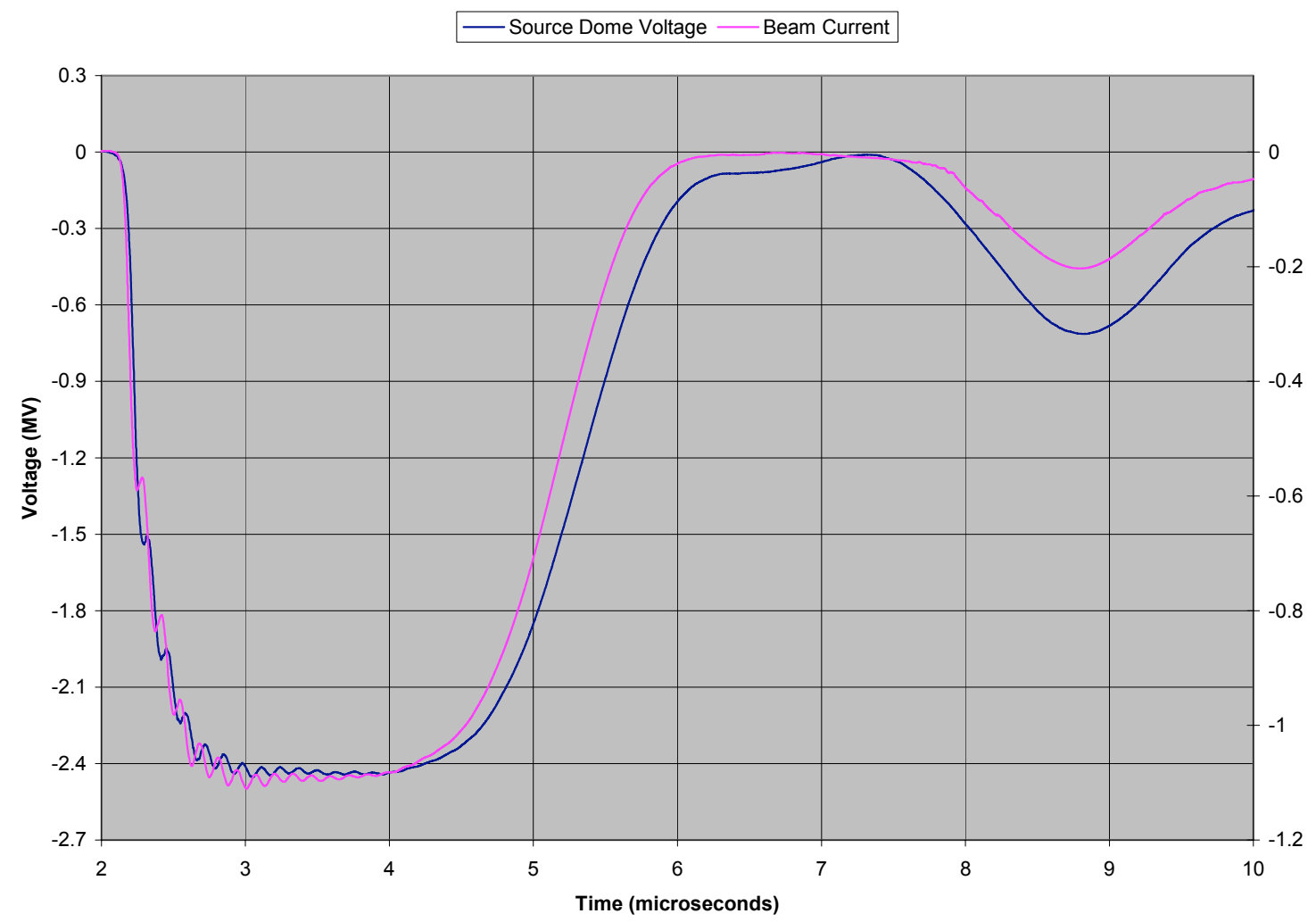

Figure 3.3 Source dome voltage and beam current (right hand scale) on BPM01 over 2 to 10 microseconds showing a $600-700 \mathrm{keV}$ "secondary beam". 


\subsection{A Simple Lumped Circuit Model of the Injector}

The highly simplified circuit model of the injector shown in Figure 3.4 is capable of describing many features of its transient behavior, including the voltage and current rise times, the amplitude and decay rate of the rise time-excited $7.8 \mathrm{MHz}$ oscillations, and the amplitude and decay rate of the crowbar-excited $\sim 6.7 \mathrm{MHz}$ oscillations. It is useful for gaining insight into the parameters that control these transient features, and it also forms the basis for the design of the small scale ("hardware") simulation model constructed at LBNL to evaluate the ferrite damper discussed in Section 4.3.

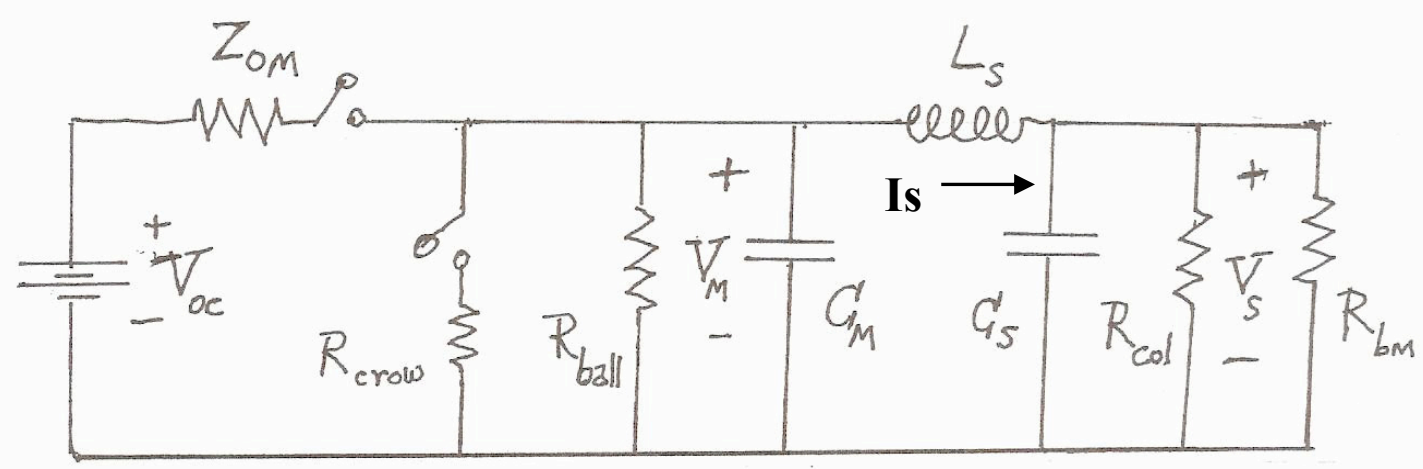

Figure 3.4 Simple circuit model of the injector.

Some definitions:

$$
\begin{aligned}
& 1 / R_{1} \equiv 1 / Z_{0 M}+1 / R_{\text {ballast }} \\
& 1 / R_{2} \equiv 1 / R_{\text {col }}+1 / R_{\text {beam }} \\
& 1 / C_{\text {series }} \equiv 1 / C_{S}+1 / C_{M}
\end{aligned}
$$

$$
\begin{aligned}
& 1 / R_{p} \equiv 1 / R_{1}+1 / R_{2} \\
& 1 / R_{L} \equiv 1 / R_{\text {ballast }}+1 / R_{2} \\
& V_{0} \equiv \frac{R_{L}}{R_{L}+Z_{0 M}} V_{o c}
\end{aligned}
$$

Note: In the flat top, $V_{S}=V_{M}=V_{0}$ is the quantity directly measured

In this circuit, the Marx PFN is represented by a simple voltage source with its characteristic impedance $Z_{0 M}$ and an instantaneous output switch. Other elements are similar to the C-L-C circuit model derived by Nielsen, McCuistian, and Kirby to describe the 7.8 MHz oscillations, namely an equivalent Marx dome and lower stalk capacitance $C_{M}$, an equivalent source dome and upper stalk capacitance $C_{S}$, and an equivalent stalk inductance $L_{S}$ [2]. The actual value of the ballast resistor is used, and the beam is represented by an equivalent linear resistor to give the correct flat-top beam current. The 
crowbar is included as an instantaneous switch with the actual value of the crowbar resistor in series (the crowbar transients will not be discussed in this section, however).

The column is represented by a single $5.3 \mathrm{~K} \mathrm{ohm} \mathrm{resistor} \mathrm{to} \mathrm{give} \mathrm{its} \mathrm{correct} \mathrm{asymptotic}$ contribution to the steady stalk current. However, the "distributed R-C network" character of the column discussed in more detail in Section 3.4 and Ref. 3 means that both the rise time transients and the equivalent impedance at $\sim 8 \mathrm{MHz}$ is very poorly modeled in this approximation. For example, the measured column current shown in Figure 3.2 jumps up at the front of the pulse and dies out in time. If the column behaved as a simple lumped resistor, the current through it would follow the waveform of the source dome voltage, i.e., it would look similar to the beam current trace in Figure 3.2. We will generally account for this aspect in making comparisons with the data. As far as the $7.8 \mathrm{MHz}$ oscillations are concerned, this approximation is not a serious limitation since it is a small contribution anyway to the damping of these oscillations [3].

The eigenfrequencies of this simple circuit contain the dominant rise time transients that are observed. With a time dependence $\sim \exp (\mathrm{st})$, the approximate solution for the three eigenfrequencies, assuming the decay rates are small compared to $1 / \omega_{0}$ (a very good approximation), is as follows.

$s_{1} \equiv-\alpha_{r}$ where $1 / \alpha_{r}=\tau_{r} \approx R_{P}\left(C_{S}+C_{M}\right)$ is the basic voltage rise time.

$s_{2,3} \equiv-\alpha_{d} \pm j \omega_{0} \quad$ where $\omega_{0}^{2}=1 / L_{s} C_{\text {series }}$ is the $7.8 \mathrm{MHz}$ oscillation, and the damping rate is defined as $\alpha_{d} \equiv \alpha_{M}+\alpha_{S}$ with

$1 / \alpha_{M}=2 R_{1} C_{M}\left(1+C_{M} / C_{S}\right)$, representing the "loading" on the Marx dome side.

$1 / \alpha_{S}=2 R_{2} C_{S}\left(1+C_{S} / C_{M}\right)$, representing the "loading" on the source dome side.

Approximate analytic solutions for the dome voltages and stalk current can be obtained in terms of these eigenfrequencies if we assume an instantaneous switching time at $\mathrm{t}=0$ for the equivalent Marx PFN source.

Initial conditions: $V_{S}, \frac{d V_{S}}{d t}, I_{S}, \frac{d I}{d t}$ all vanish at $\mathrm{t}=0$.

A final condition is the asymptote $V_{S}(t \rightarrow \infty)=V_{0}$. 
The solutions using these initial conditions and final condition are:

$$
\begin{aligned}
& V_{S}(t)=V_{0}\left[1-\exp \left(-\alpha_{r} t\right)-\frac{\alpha_{r}}{\omega_{0}} \sin \left(\omega_{0} t\right) \exp \left(-\alpha_{d} t\right)\right] \\
& V_{M}(t)=V_{0}\left[1-\exp \left(-\alpha_{r} t\right)+\frac{\alpha_{r}}{\omega_{0}} \frac{C_{S}}{C_{M}} \sin \left(\omega_{0} t\right) \exp \left(-\alpha_{d} t\right)\right] \\
& I_{S}(t)=I_{0}\left[\exp \left(-\alpha_{r} t\right)-\cos \left(\omega_{0} t+\delta\right) \exp \left(-\alpha_{d} t\right)\right]+\frac{V_{0}}{R_{2}}\left[1-\exp \left(-\alpha_{r} t\right)\right]
\end{aligned}
$$

where $I_{0} \equiv \alpha_{r} C_{S} V_{0} \quad$ and $\delta \sim \alpha_{r} / \omega_{0} \ll 1$

In the remaining parts of this subsection we will compare various predictions of this simple model with data from two representative shots obtained during the Injector Working Group conclave in July 2005.

\subsubsection{Parameter Selection}

In a simplified equivalent circuit like the one we are using here, a small number of circuit parameters are chosen to best match the electrical features of the actual multi-parameter distributed structure. These can of course be estimated from the physical structure itself, as originally done by Nielsen et al. [2]. Here we will "fine tune" these parameter estimates by using a few "data peg points" to assure that key features like the frequency of the dominant $7.8 \mathrm{MHz}$ oscillations are accurately contained in the model. The main motivation for this "fine tuning" is to assure that the scaled hardware model described in Section 4.3 reproduces the actual injector transients as accurately as possible. 


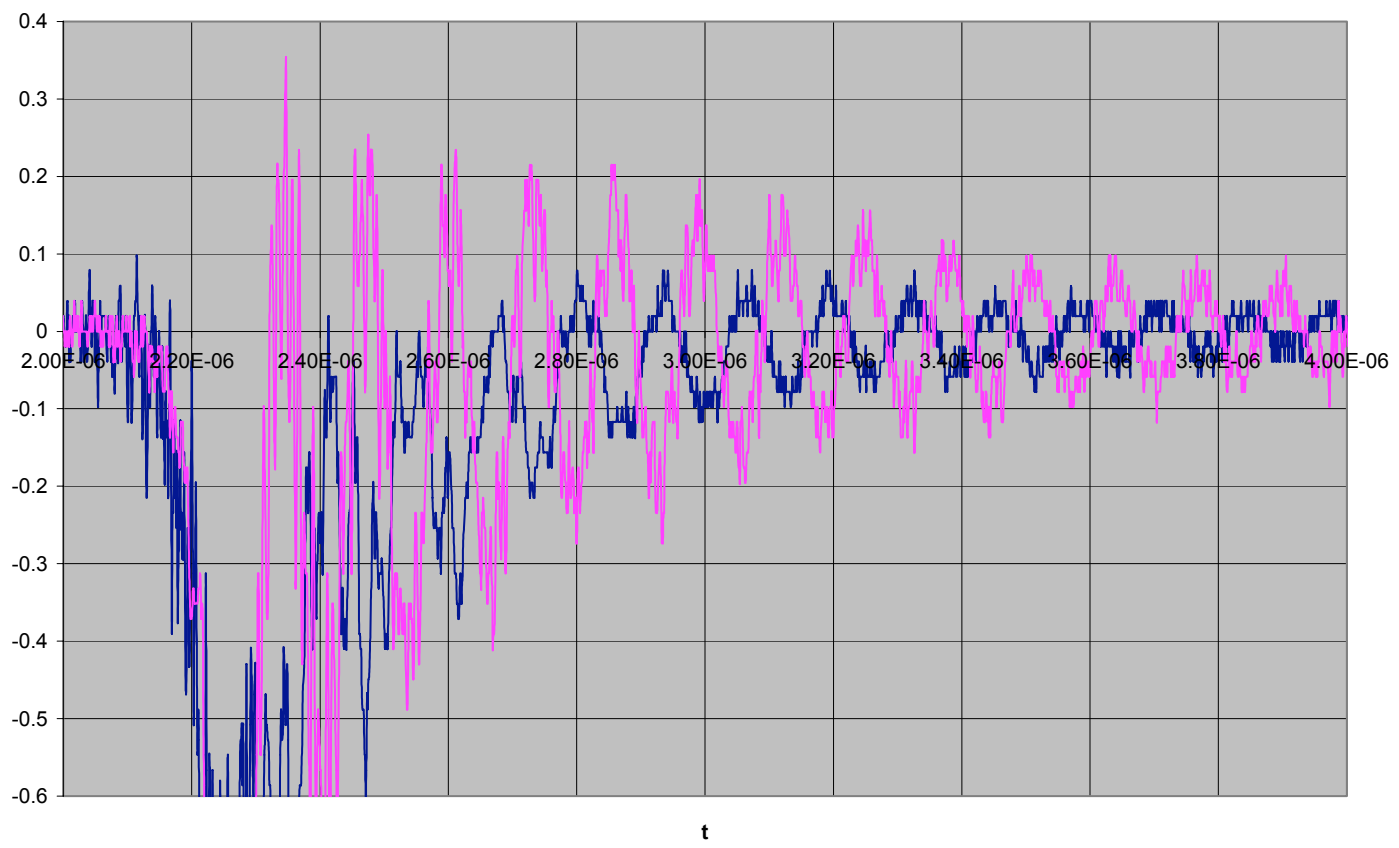

Figure 3.5 Marx dome and source dome E-Dot signals.

The first "peg point" is the ratio of the 7.8 MHz E-Dot signal amplitudes on the source dome to the Marx dome with the no-beam shot data (\#1897) shown in Fig 3.5. We see from Eq.s (3.1) and (3.2) that this ratio (2.4 after accounting for the calibrations of both) determines the ratio

$$
C_{M} / C_{S}=2.4
$$

The second "peg point" is the voltage rise time with the same no-beam shot (\#1897). The fit shown in Figure 3.6 gives the exponential time constant

$$
\tau_{r}=1 / \alpha_{r}=0.23 \mu \mathrm{sec}
$$

This determines the product

$$
R_{P} C_{S}=6.76 \times 10^{-8} \mathrm{sec}
$$

where $R_{P}$ is the parallel combination of the ballast resistor (912 ohms on this shot), the $5.3 \mathrm{~K}$ ohm column resistor, and $Z_{\text {ом }}$ (to be determined).

The final "peg point" is assuring an oscillation frequency of $7.8 \mathrm{MHz}$. Using the ratio $C_{M} / C_{S}=2.4$ already determined, this condition requires

$$
L_{S} C_{S}=5.9 \times 10^{-16}
$$

If we select a value for the stalk inductance (say), the rest of the parameters are now determined. The value for stalk inductance estimated from the physical structure is 2.7 microhenries [2]. This choice leads to the first parameter set in the Table 3.1. 


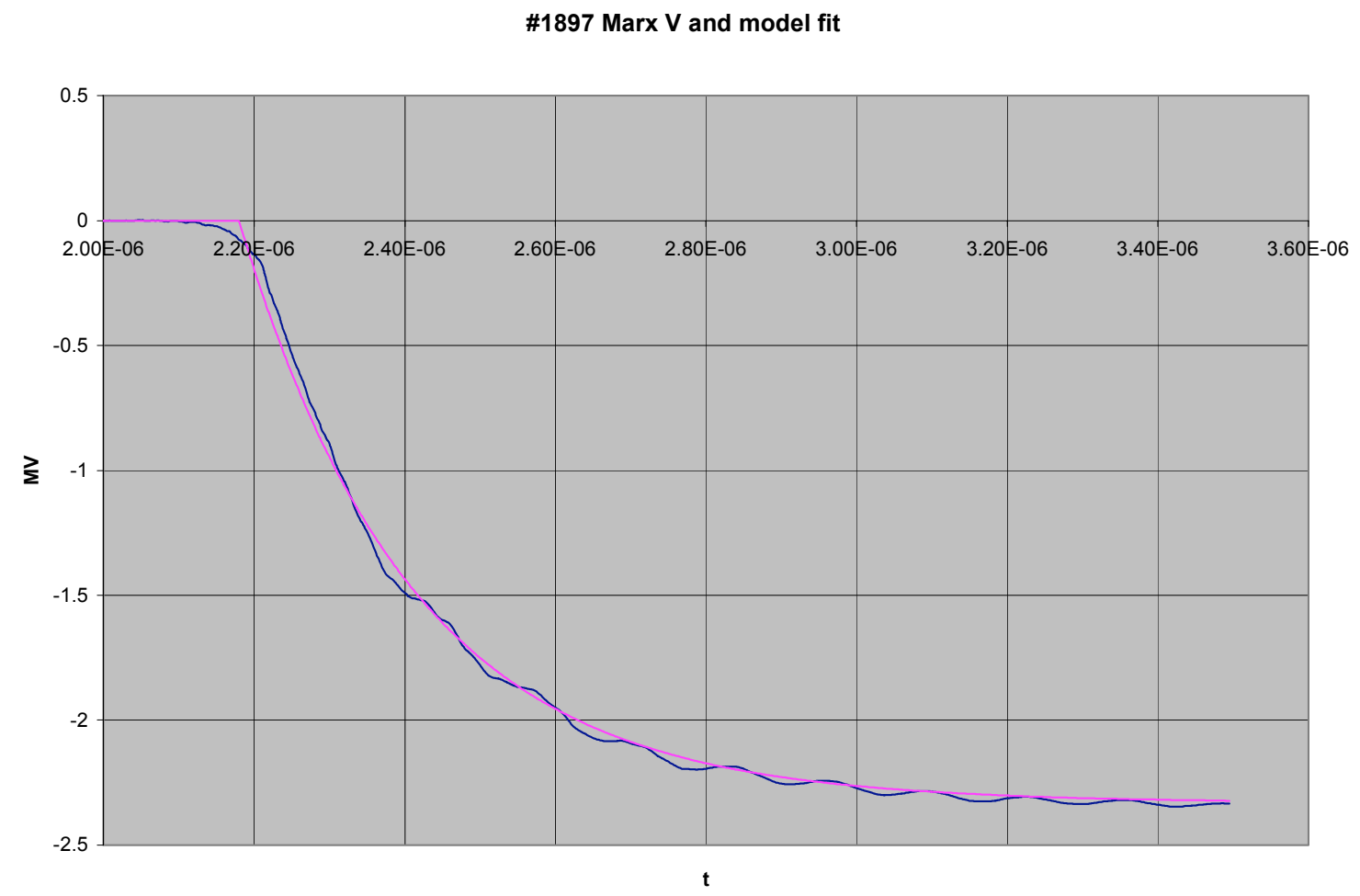

Figure 3.6 Marx dome $V(t)$ rise time and exponential fit.

\begin{tabular}{||l|l|l|l|l||}
\hline \hline Circuit element & $C_{S}$ & $C_{M}$ & $L_{S}$ & $Z_{0 M}$ \\
\hline Set \# 1 & $220 \mathrm{pF}$ & $528 \mathrm{pF}$ & $2.7 \mu h$ & $510 \mathrm{ohms}$ \\
\hline Set \# 2 & $200 \mathrm{pF}$ & $480 \mathrm{pF}$ & $3 \mu h$ & $600 \mathrm{ohms}$ \\
\hline
\end{tabular}

Table 3.1

The "effective" Marx impedance $Z_{0 M}$ in parameter set $\# 1$ is $\sim 20 \%$ lower than is generally quoted (640 ohms). The "rounded off" parameter set \#2 ( 10\% "fine tuning" in the C's and the L) has a somewhat higher $Z_{0 M}$, and the effective source end capacitance is also not unreasonable ( $140 \mathrm{pF}$ for the dome itself, plus $60 \mathrm{pF}$ for the upper $1 / 2$ of the stalk). For no particularly compelling reason, we used parameter set \# 2 as the design basis for the ferrite damper simulation circuit (scaled to 1/10 impedance levels, preserving the rise time, oscillation frequency and damping rate).

\subsubsection{Excitation Level of the 7.8 MHz Oscillations}

The simple circuit model with an assumed "instantaneous" Marx PFN rise time predicts that the excitation level of the $7.8 \mathrm{MHz}$ voltage fluctuation on the source dome is $\alpha_{r} / \omega_{0}=1 / \omega_{0} \tau_{r}$ times the flat top voltage level, see Eq. (3.1). A sensitive test of this 
prediction is provided by a comparison of the raw source dome E-Dot signal with our model, using the derivative of Eq. (1) to obtain

$$
\frac{d V_{S}}{d t}=\alpha_{r} V_{0}\left[\exp \left(-\alpha_{r} t\right)-\cos \left(\omega_{0} t\right) \exp \left(-\alpha_{d} t\right)\right]
$$

Bdot3 and fit

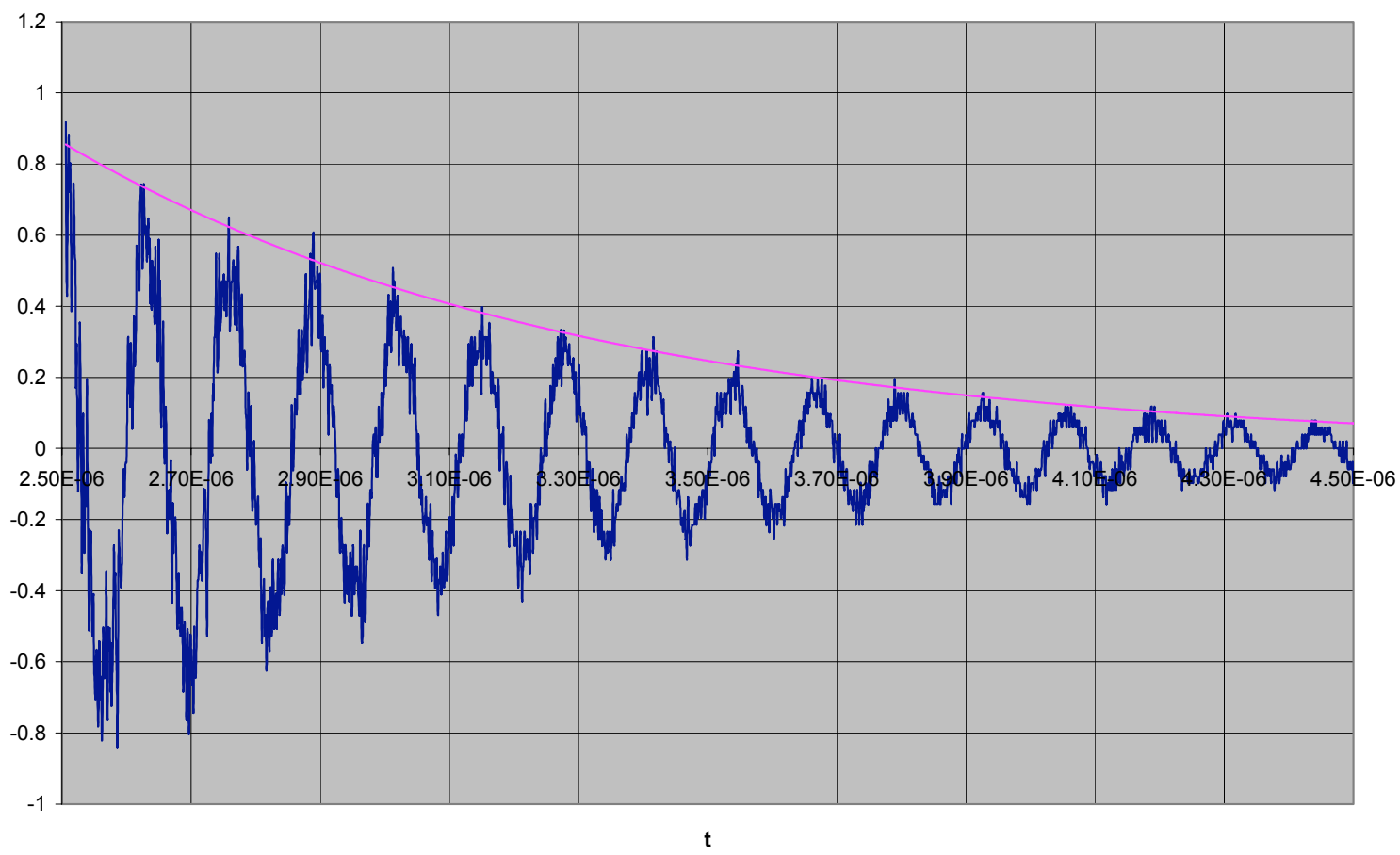

Figure 3.7 B-Dot \#3 on shot \# 1897 and the fit to an exponential.

The B-Dot \#3 signal on shot \#1897 provides the cleanest measure of the oscillation damping rate and frequency (needed to compare the Eq. (3.4) theoretical waveform with the E-Dot data). This data (see Figure 3.7) fits an oscillation damping time constant of $\tau_{d}=0.8 \mu \mathrm{sec}$ and a frequency $7.7 \mathrm{MHz}$. 
Edot source dome and theory

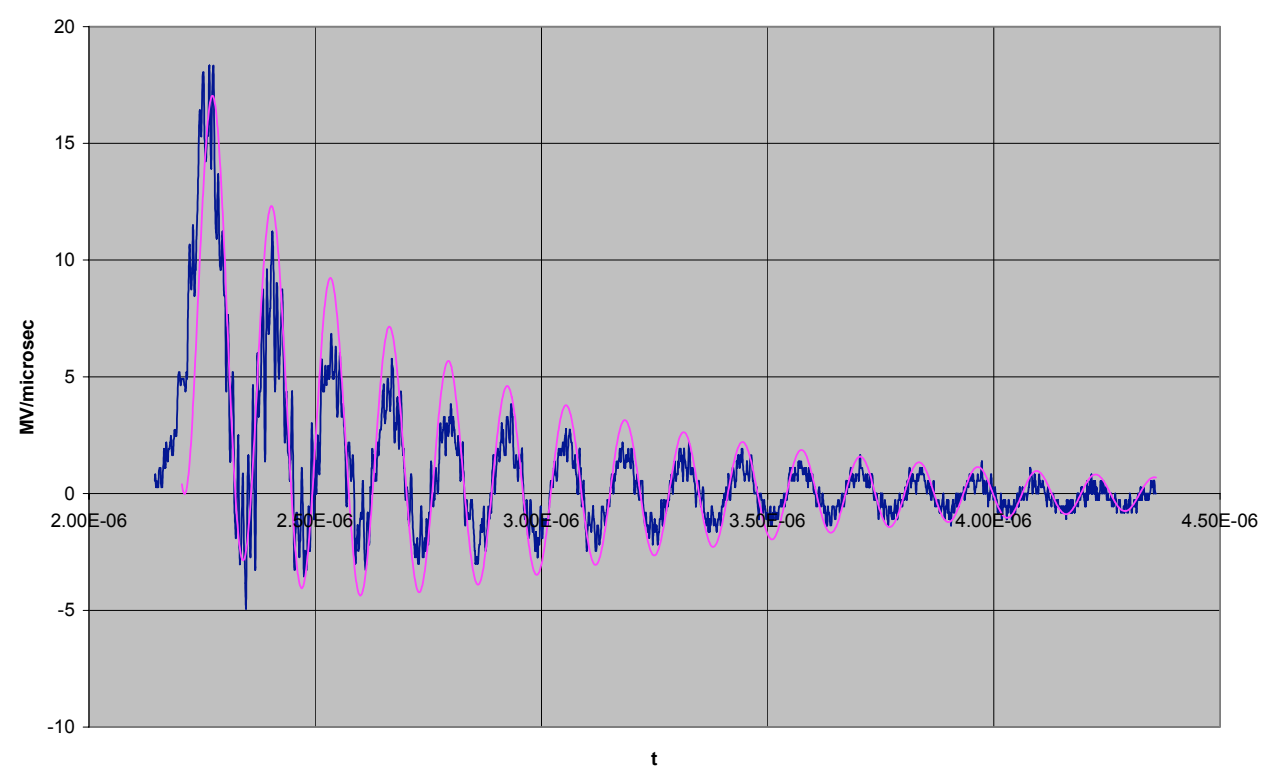

Figure 3.8 Comparison of raw source dome E-Dot signal (x14) with the circuit model prediction in Eq. (3.4).

Using these parameters, the observed flat top voltage of $2.33 \mathrm{MeV}$, and the observed voltage rise time of $\tau_{r}=0.23 \mu \mathrm{sec}$ in Eq. (3.4), we have the comparison of the model with the source dome E-Dot shown in Figure 3.8 for shot \#1897 (the units are $\mathrm{MeV} /$ microsecond for both).

The agreement of the simple circuit model formula with the data is actually quite remarkable (even accounting for the use of experimental values for $V_{0}, \tau_{r}, \tau_{d}$ ). The observed source dome voltage has a more gentle turn on at the beginning than the model, as expected, since the Marx PFN has a finite rise time. But the amplitudes of both the 7.8 $\mathrm{MHz}$ oscillations and the exponential term in the source dome voltage are as predicted.

\subsubsection{Damping Rate of the 7.8 MHz Oscillations without Beam}

Using the circuit model parameters in set \# 2, the contribution to the damping rate of the 7.8 $\mathrm{MHz}$ oscillations from the Marx dome "loading" is predicted to be $1 / \alpha_{M}=1.18 \mu \mathrm{sec}$, while the source dome "loading" from the column resistor.

gives $1 / \alpha_{S}=2.8 \mu \mathrm{sec}$. So the net damping time is predicted to be $\tau_{d}=1 / \alpha_{d}=0.83 \mu \mathrm{sec}$, in reasonable agreement with the observed value of $0.8 \mu \mathrm{sec}$.

As we discussed earlier, the contribution of the lumped element column resistor in the model to this damping is actually an overestimate, so this close agreement is somewhat superfluous. For example, the EM model in Ref. 3 indicates that the distributed column resistors contribute a damping time of $9 \mu \mathrm{sec}$; using this for $1 / \alpha_{S}$ gives a net damping time of $1.04 \mu \mathrm{sec}$. (Addition of other damping sources, for example an effective series 
resistance of 1.8 ohms to the stalk inductance, would then be needed to bring the prediction back in line with the observation.)

\subsubsection{Voltage Rise Time and 7.8 MHz Oscillation Damping Rate with a $1.2 \mathrm{kA}$ Beam}

As another test of the model's predictive capability, we compare it with data from a shot producing a $1.2 \mathrm{kA}$ beam (shot \# 1900). The voltage rise time observed in this case is $\tau_{r}=0.19 \mu \mathrm{sec}$ (see Figure 3.9), $\sim 17 \%$ faster than the previous no beam case. If we use an equivalent beam resistance of 2 kilo-ohms, the circuit prediction is $\tau_{r}=0.196 \mu \mathrm{sec}$.

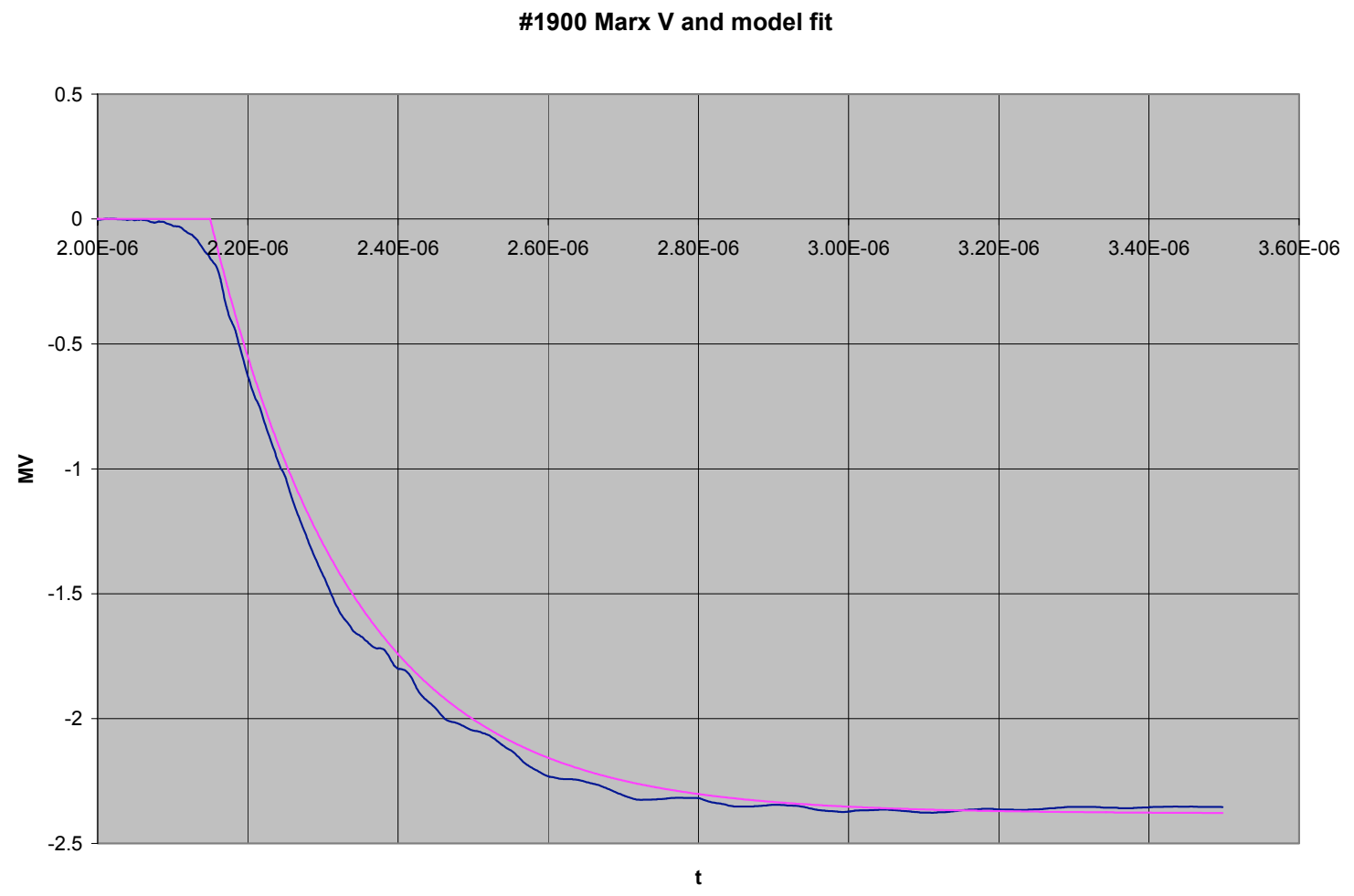

Figure 3.9 Marx dome voltage rise time and exponential fit for shot \#1900 (1.2 kA beam). 


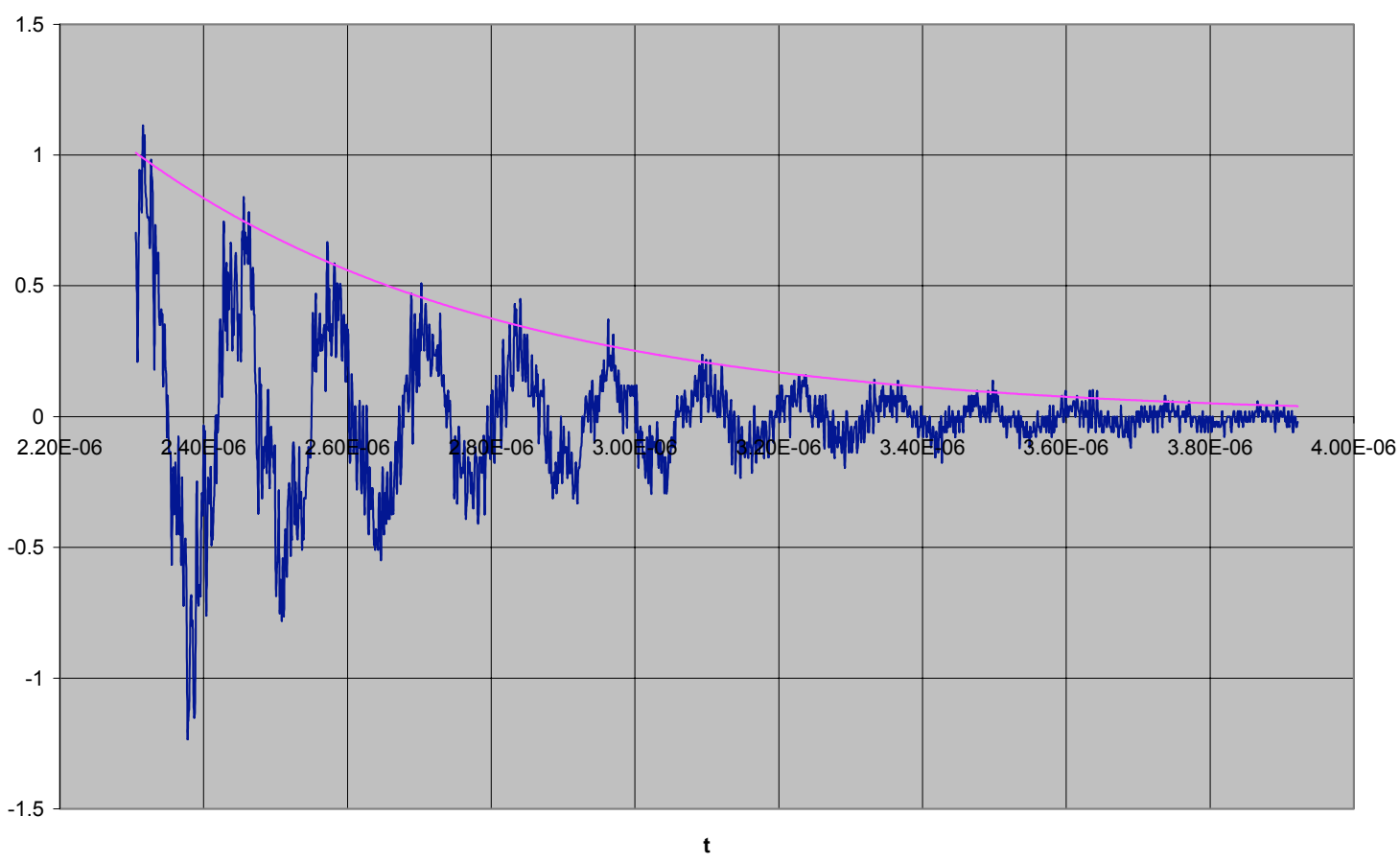

Figure 3.10 B-Dot \#3 data and exponential fit for shot \# 1900 (1.2 kA beam).

The damping rate of the $7.8 \mathrm{MHz}$ oscillations is considerably faster with a beam, as has been noted many times before by the DARHT experimental team. The B-Dot \#3 loop signal for this shot along with a fit to an exponential decay is shown in Figure 3.10. The observed damping rate in this case is $\tau_{d} \approx 0.5 \mu \mathrm{sec}$, a damping rate about $60 \%$ faster than the no beam case.

The predicted time constant from the circuit using a 2 Kohm resistor to represent the beam is $0.41 \mu \mathrm{sec}$. As in the "no beam" case, using the single lumped element model of the column resistor in the circuit overestimates its contribution to the damping. Using the EM simulation results for the contribution of the distributed column resistors, the net damping time is predicted to be $0.54 \mu \mathrm{sec}$. 


\subsection{Column Rise Time Transients}

The electrical stress on the insulating column has always been one of the areas of concern in the injector. The time dependence of the stress on the column in the rise time phase was modeled, and this modeling was the basis for the tapering of the grading resistor values in each of the sections that was chosen. The objective of the present subsection is to summarize the operational data and the subsequent modeling of these rise time transients. The transients associated with operation of the crowbar are discussed in the next subsection.

A resistively-graded potential profile with constant current flowing down the column is obtained in the "steady state" after all the transients are over. Early in the pulse, however, large displacement currents are required to charge up the capacitance between the column and the stalk, particularly near the bottom where the voltage across them is the greatest. With a stalk-to-column capacitance around $100 \mathrm{pF} /$ meter, a column-to-tank capacitance around $45 \mathrm{pF} / \mathrm{meter}$, and a resistance per 0.4 meter section ranging from 250 to $700 \mathrm{ohms}$ (say $\sim 1.5 \mathrm{Kohms} /$ meter average), the e-folding timescale for charging (or discharging) this distributed R-C ladder line can be estimated as

$$
\tau_{\text {column }} \sim(145 p f / m)(1.5 K \Omega / m) l^{2} \sim 0.3-0.6 \mu \mathrm{sec}
$$

where we used a characteristic length in the diffusion equation of $\sim 1-2$ meters $(1.8 \mathrm{~m}$ is half the column length for example). The main point of this estimate is to show that the timescale for "redistribution" of the column voltage profile from capacitive-graded to resistively-graded is longer than the source dome voltage rise time.

A comprehensive end-to-end circuit model of the MARX and the injector-column structure has been developed by Mike Kang (an early version is described in Ref. [4]). This model includes the voltage dependence of the column resistors and the MOV's on the column. To illustrate the predictive capability of this model, a comparison of the source dome voltage for shot \# 1660 with the model is shown in Figure 3.11. 


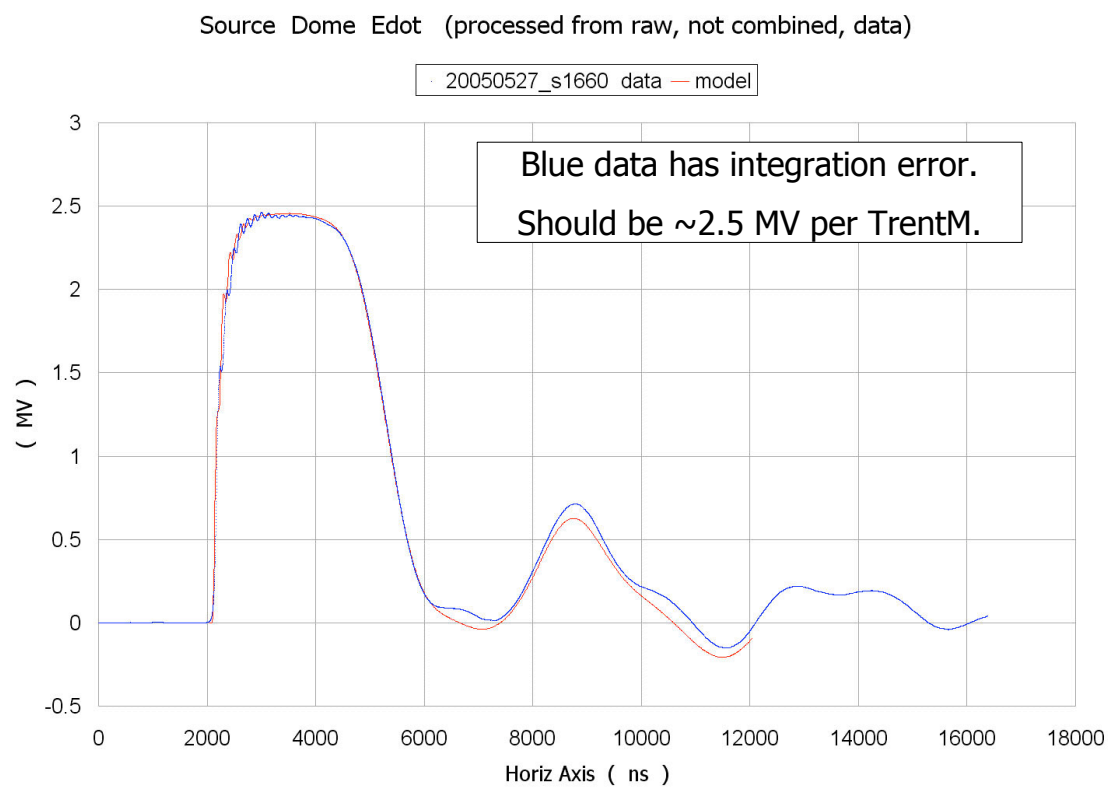

Figure 3.11 Source dome voltage for shot \#1660 and the end-to-end model.

In Figure 3.12, the model's prediction of the voltages across each of the lowest four sections of the column during the first 2 microseconds is presented. The grading resistor values of the lowest four sections (in round numbers) are 250, 350, 450, and $700 \mathrm{ohms,}$ respectively, moving up the column. Note that the voltage of the lowest section rises rapidly at the front, and then decays to its steady-state value in around one microsecond (a timescale consistent with the simple $\mathrm{R}-\mathrm{C}$ diffusion estimate given above). The initial rise of the lowest section's voltage, more than twice its steady state value, is due to the large capacitive current flowing to the lower part of the column as the stalk voltage rises, returning back down the column through the resistors. 


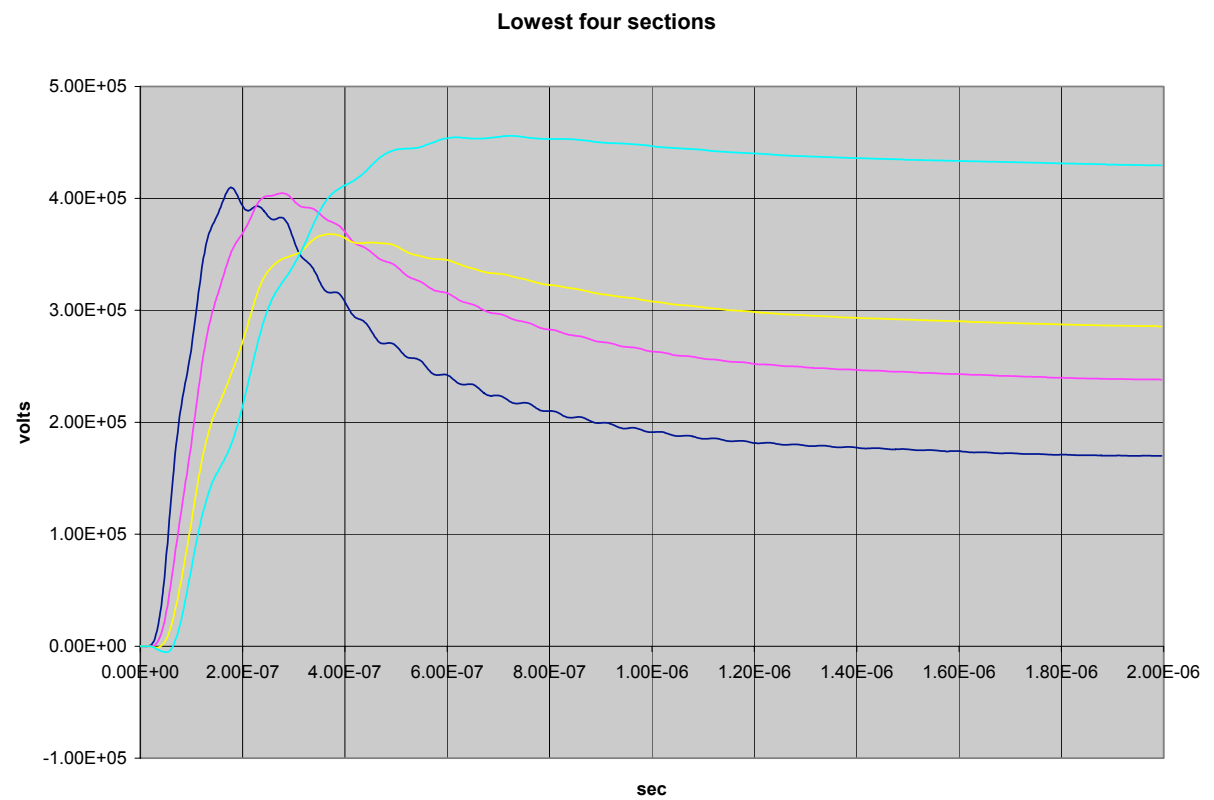

Figure 3.12 Voltage waveforms on the bottom four column sections. Column section 1 (bottom) is the blue line, 2 is the magenta line, 3 the yellow line, and 4 the green line.

In Figure 3.13, the voltage waveforms on the upper five sections predicted by the model are shown. The grading resistors here are all $700 \mathrm{ohms}$ per section. Note that the $7.8 \mathrm{MHz}$ oscillations are clearly evident on the upper three sections, but not on the others.

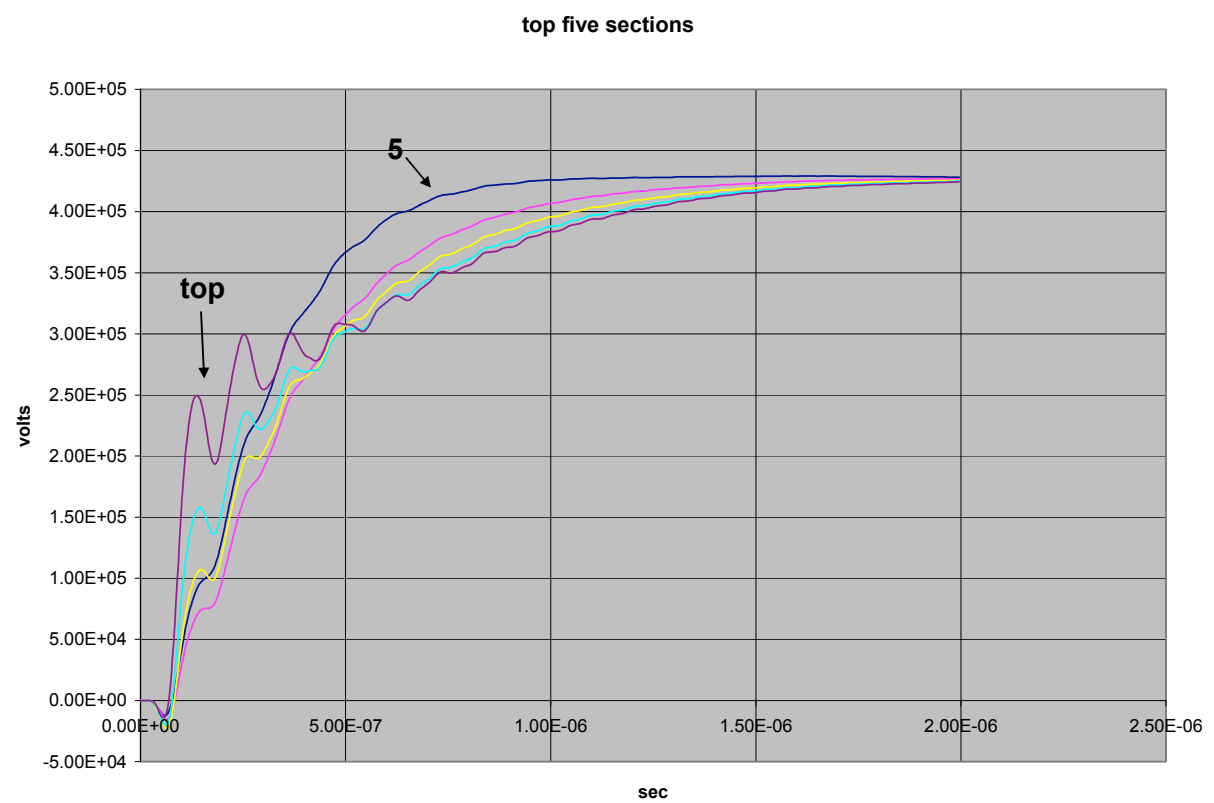

Figure 3.13 Voltage waveforms on the top five column sections. The top section (\#9) is the purple line (indicated), \#8 is the green line, \#7 the yellow line, \#6 the magenta, and \#5 the blue line (indicated). 
Note that the peak voltages of every section predicted by Mike Kang's model never exceed $\sim 450 \mathrm{KV}$, and at a source dome voltage level $\sim 2.5 \mathrm{MeV}$ none of the MOV's are predicted to turn on (Some do briefly at the $3.2 \mathrm{MeV}$ level). The column resistor tapering prescription adopted in the original design therefore does appear to have been a very good choice based on this more recent and elaborate model.

The final "validation" needed is comparison with the data of course. The predicted current at the bottom of the column is compared with the column CVR data for shot \# 1660 in Figure 3.14. The agreement is excellent both in the peak amplitude of the front "spike" and in the wave shape.

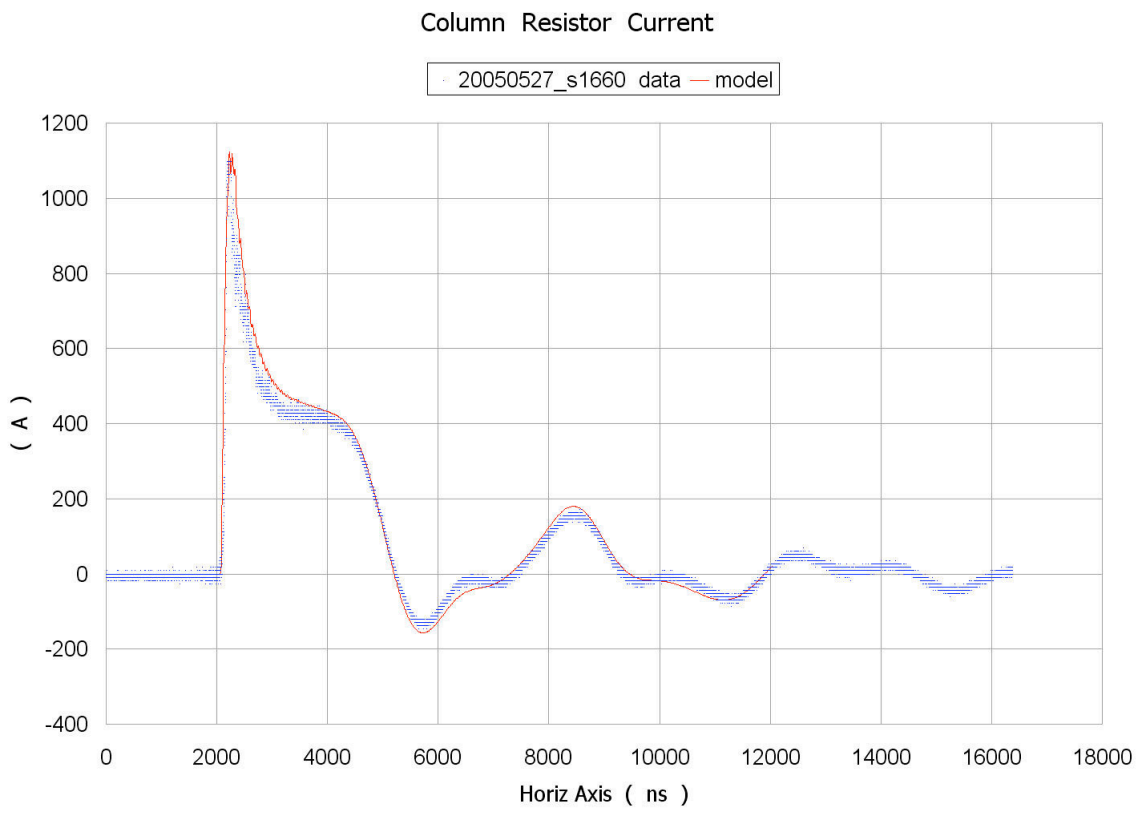

Figure 3.14 Comparison of the current at the bottom of the column predicted by the model with the column CVR data for shot \# 1660.

\subsection{Crowbar Operation}

The crowbar has not been used for more than a year because of various concerns. In this subsection we will attempt to describe the key observations, and outline the understanding that has been achieved. The "story" is more complicated here, unfortunately, because at the time the crowbar was operated many of the injector diagnostic signals were severely clipped following the crowbar firing, and the key column current diagnostic was compromised because the MOV and resistor chains were miswired. 
The source dome voltage on a "typical" crowbar shot (\#1122) is shown in Figure 3.15. When the crowbar fires, large amplitude oscillations in the source dome voltage are induced. The frequency of these oscillations, around $6.7 \mathrm{MHz}$, is lower than the rise time oscillations. We would expect this from the equivalent circuit in Figure 3.4. If the crowbar resistor fully shorted out the Marx dome capacitance, the resonant frequency would be given by $\omega_{0}=\left(L_{S} C_{S}\right)^{-1 / 2}$, or $f_{0} \sim 6.5 \mathrm{MHz}$. The damping of the oscillations is also much stronger, again in general accord with what one expects from the equivalent circuit with $\mathrm{a} \sim 30 \mathrm{ohm}$ crowbar resistor in series with the stalk inductance. Finally, the level the source dome voltage approaches as the oscillations die out after the crowbar firing should be determined by the ratio of crowbar resistance to Marx impedance multiplied by $V_{0 C}$ (see the definitions in Figure 3.4), in reasonable accord with the observed value of $\sim 0.25 \mathrm{MeV}$.

Source dome V(t), shot \#1122

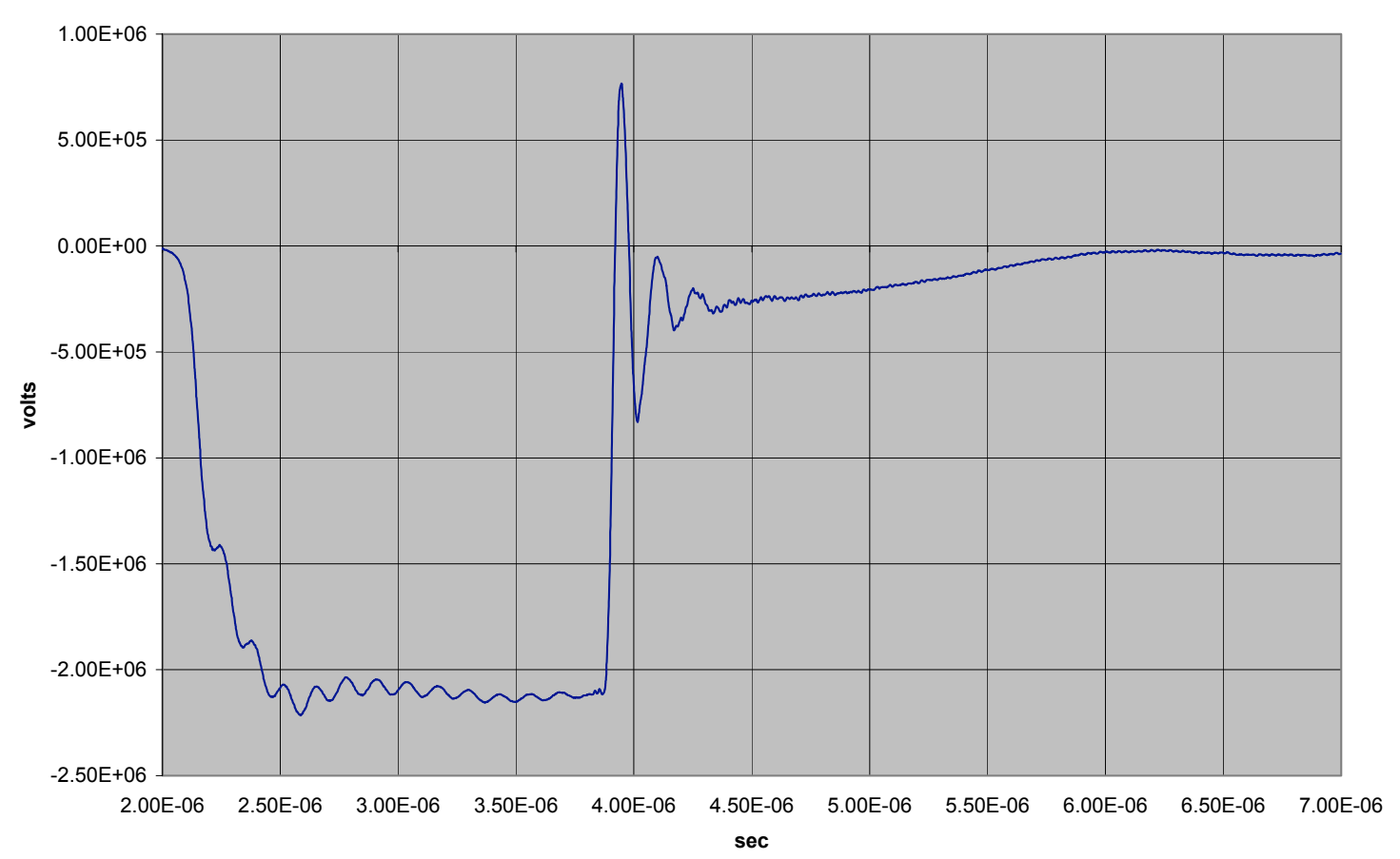

Figure 3.15 Source dome voltage on a crowbar shot \# 1122 (compare with the shot without a crowbar in Figure 3.1).

The amplitude of the crowbar-induced $6.7 \mathrm{MHz}$ oscillations is much larger than the rise time induced $7.8 \mathrm{MHz}$ oscillations, again in accord with the simple circuit model. As a result, the source dome voltage reverses sign and swings positive (to $\sim+750 \mathrm{keV}$ in the data shown). This has apparently been an area of concern, with an inference being made that it is related to stress reversal on the column. Our discussion of the column transients in the previous subsection, however, should make it clear that the column voltage distribution is not related in a simple way to the instantaneous source dome voltage. 
Based on our review of the crowbar data base in the Injector Working Group, the very large amplitude crowbar-excited oscillations at higher frequencies observed on other diagnostics is an area of much greater concern. For example, consider the key diagnostic that gives direct information about the column stress, namely, the CVR at the bottom of the column. The shot \#1122 "MOV" and "Resistor chain" CVR's are shown in Figure 3.16. (Due to the miswiring, both signals are a combination of the two different types of chain).

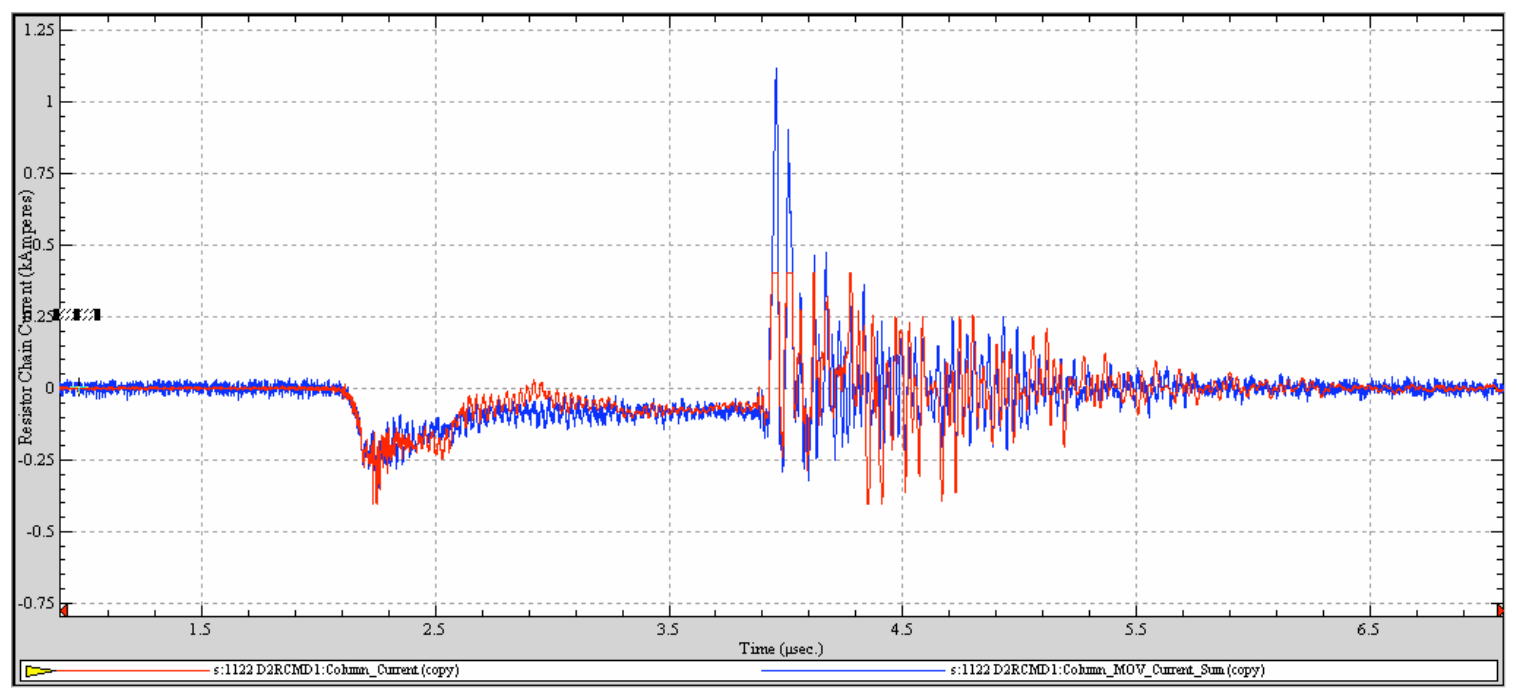

Figure 3.16 Column CVR for crowbar shot \# 1122. Both traces are basically single resistive string currents; the blue string current was not clipped (absolute calibration is in question, but the total column current in the "flat" region should be $\sim 400 \mathrm{amps}$ ). 


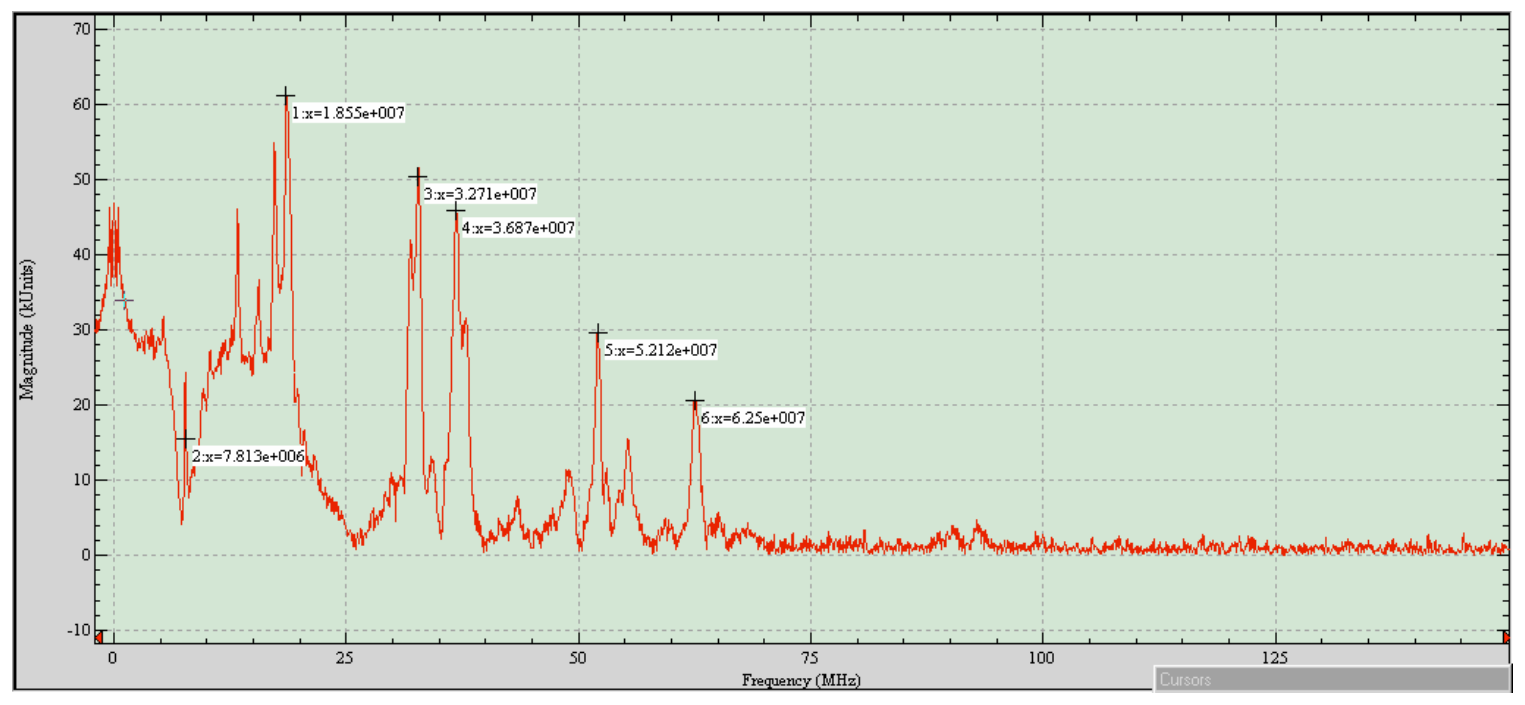

Figure 3.17 FFT of the column CVR for shot \# 1122 in the time window after crowbar firing.

FFT's of this column CVR after crowbar firing are shown in Figure 3.17 using the blue trace in Figure 3.16 (the one that was not clipped).

One striking thing about this frequency spectra is that the strongest signal on the CVR at the bottom of the column is at $\sim 18-19 \mathrm{MHz}$ (a frequency that is also very apparent when looking at the original signal on an expanded time base). The $6.7 \mathrm{MHz}$ signal is almost non-existent, while a number of even higher frequency peaks appear up to $\sim 60-70 \mathrm{MHz}$.

The other striking thing is the amplitude of these oscillations. The positive "spike" on the CVR signal in Figure 3.16 is at least ten times the "steady level" before the crowbar fires (this steady asymptote should be of order $2.1 \mathrm{MeV} / 5 \mathrm{Kohms} \sim 400 \mathrm{amps}$, or $\sim 100 \mathrm{kV}$ on the lowest column section). So a peak pulse voltage on the lowest section of order one megavolt or larger lasting for 10-20 nanoseconds is indicated by this data.

Given the uncertainties and general "background noise level" (real or not?) in the column CVR signal, it is useful to also look at the B-Dot signals. Clipping was a serious limitation here also. B-Dot \# 1 (near the top of the tank) had minimal clipping, so it was used for the FFT in the time window after crowbar firing shown in Figure 3.18. With this diagnostic, the strongest resonances are the low Q 6.7 $\mathrm{MHz}$ oscillations seen so clearly also on the source dome voltage, and the $\sim 19 \mathrm{MHz}$ signal seen so clearly on the column CVR. Additional higher frequency resonances are apparent at $32 \mathrm{MHz}$ and $53 \mathrm{MHz}$. 


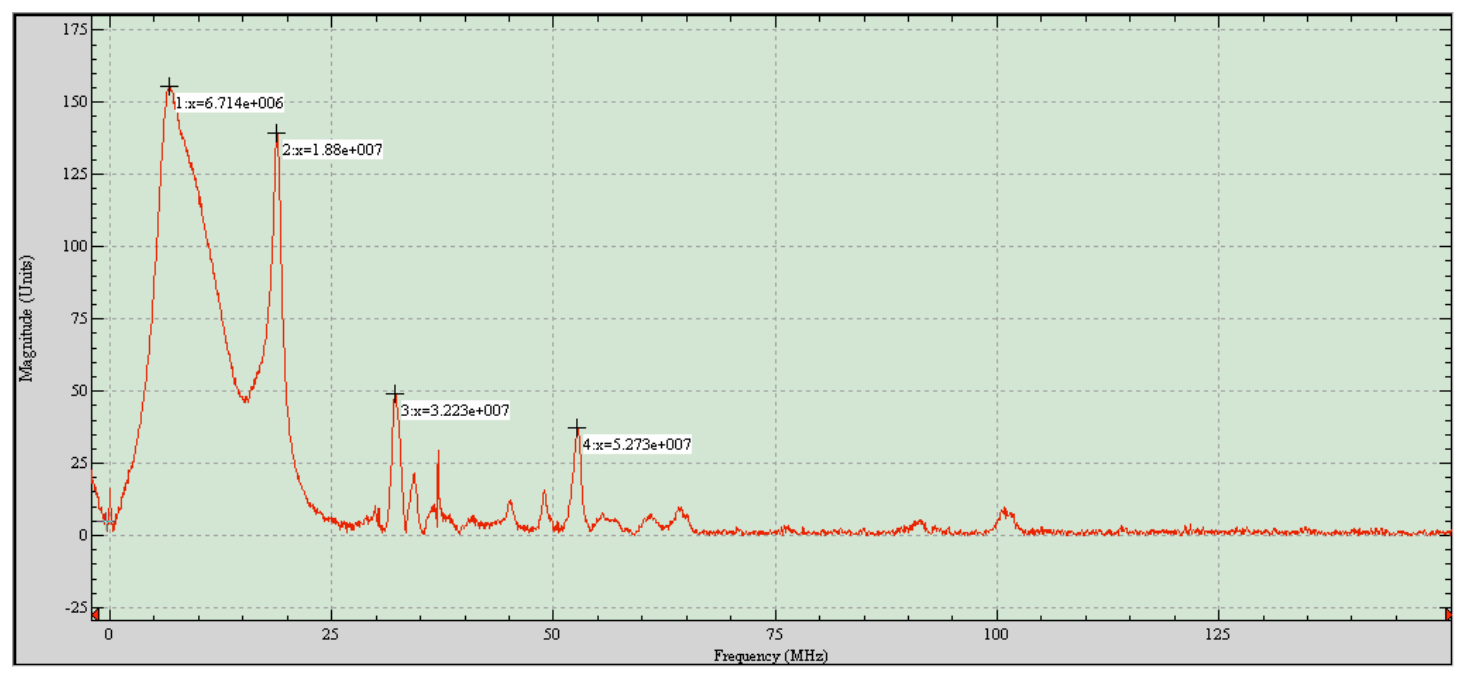

Figure 3.18 FFT of B-Dot \#1 in the time window after crowbar firing for shot \# 1122.

To provide additional insight into these oscillation modes, it is useful to also look at the FFT spectrum of shots without a crowbar, or equivalently, at the FFT of B-Dot \#1 for shot \#1122 in the time window before the crowbar is fired (see Figure 3.19). The only oscillation mode of any significance (for the injector dynamics) seen on this diagnostic is our familiar friend, the $7.8 \mathrm{MHz}$ mode (here appearing to be at $\sim 7.6 \mathrm{MHz}$ ). A "whisper" is seen of the $\sim 18 \mathrm{MHz}$ mode, and also a few small peaks at higher frequencies, even going above $100 \mathrm{MHz}$ (these might act as seeds for BBU).

In contrast, the FFT of the column CVR signal in the time window before the crowbar fires for shot \# 1122 has almost no evidence of the $7.8 \mathrm{MHz}$ oscillation (see Figure 3.20). The only small peaks seen above the 1/f background level (note the scale change compared to Figure 3.17) are around 32 and $38 \mathrm{MHz}$. 


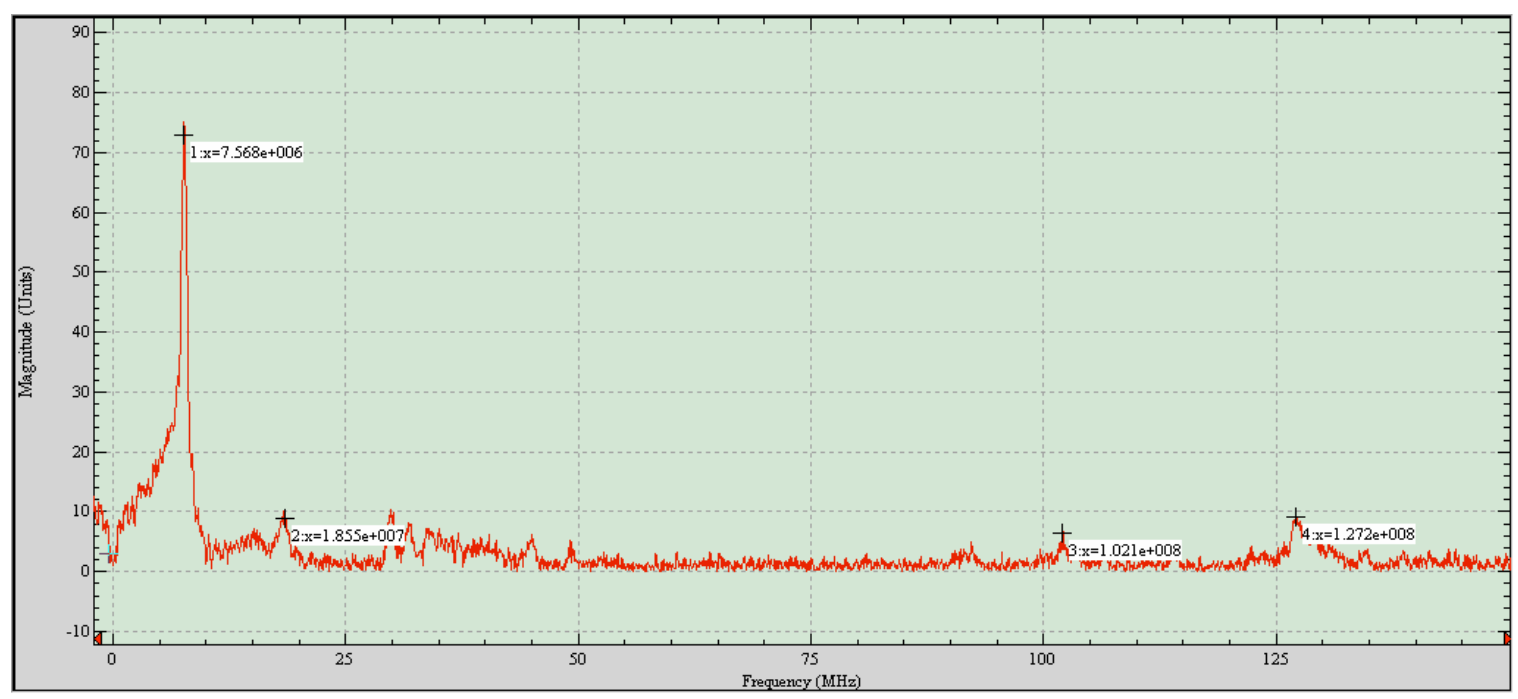

Figure 3.19 FFT of B-Dot \#1 in the time window before crowbar firing for shot \# 1122 .

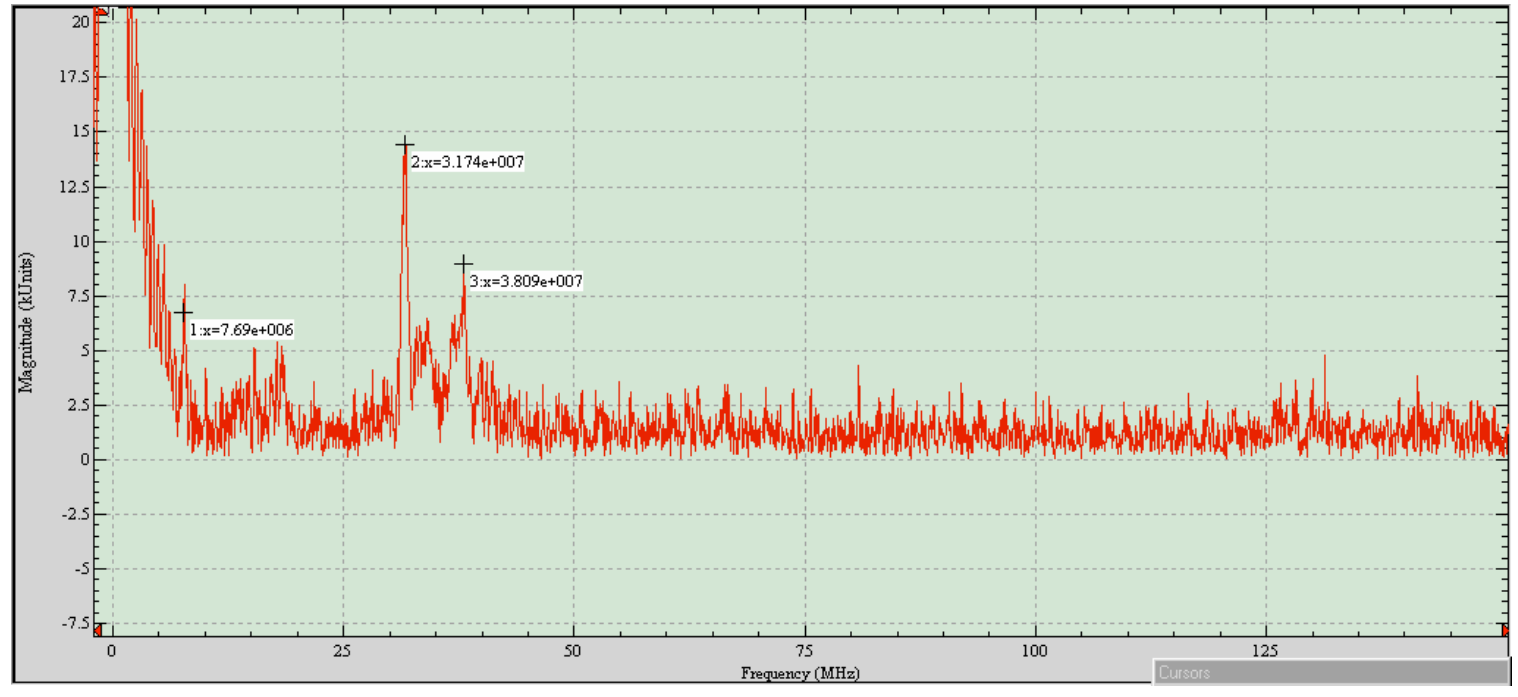

Figure 3.20 FFT of the column CVR for shot \# 1122 in the time window before crowbar firing (note the amplitude scale change here compared to the FFT after crowbar firing in Figure 3.17)

\subsubsection{EM Mode Simulations}

Henestroza, et al. have done 3-D time-dependent electromagnetic field calculations of a DARHT injector model using MAFIA [3, 5]. The injector geometry and the components used in this model are shown in Figure 3.21. These numerical simulations were all for the case before the crowbar was fired, that is, the conductive media representing the crowbar resistor was capacitively coupled from the Marx dome to the tank wall. The model also had conductive media to represent the beam and ballast, and it used the actual column 
resistor values. Here our main interest is in the field maps of some of the modes, to provide insight into the observations described above, and not in the damping rates. And even though the simulations were for the case of the crowbar in the "open state", we will assume the results are at least generally indicative of the modes after the crowbar has fired.

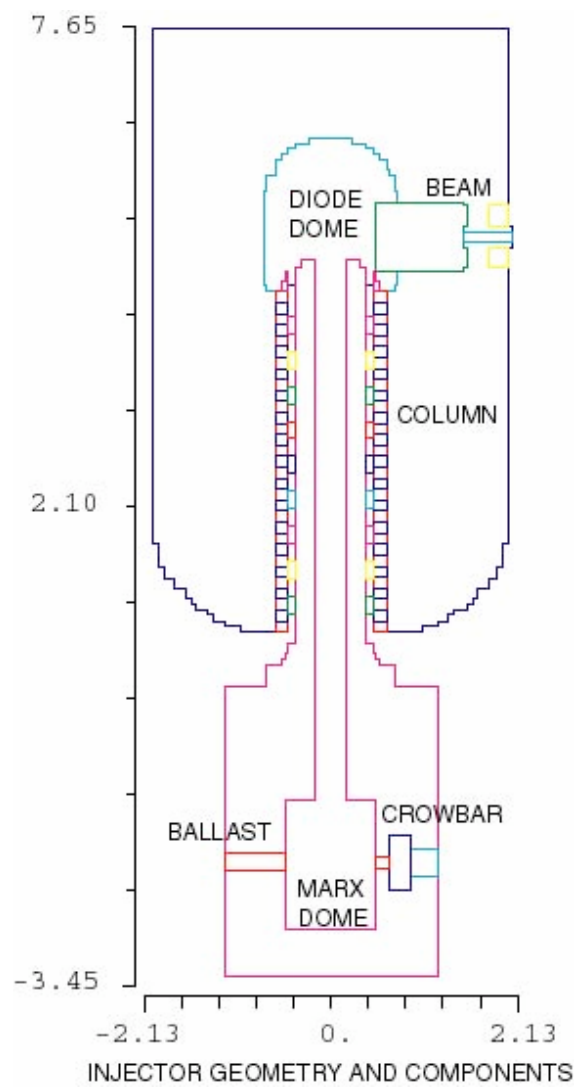

Figure 3.21 EM model of the injector.

The E-field and B-field maps of the lowest mode predicted by the model, at a frequency around $8 \mathrm{MHz}$, are shown in Figure 3.22. Note that the E-field has a null around the bottom of the column for this mode. (We can also recognize that a voltage null occurs there from the simple circuit model, by separating the stalk inductance into the upper stalk $(1900 \mathrm{nH})$ and lower stalk $(800 \mathrm{nH})$; see Ref. [3].) The magnetic field on the other hand is near its peak value in this location, which is close to where the stalk Rogowski is located.

Many aspects of the observations of this mode described in the previous subsections are clarified by this field map. The column CVR located at the bottom of the column would respond primarily to the axial RF electric field at that location, which is near a null. So the absence of $7.8 \mathrm{MHz}$ (or the related $6.7 \mathrm{MHz}$ ) oscillations on this diagnostic should not be a surprise (See Figure 3.2 and the column CVR FFT data both before and after the crowbar is fired in Figures 3.17 and 3.20). The B-Dot \#1 diagnostic, on the other hand, is located in a region where the RF magnetic field of this mode is appreciable (see Figure 
2.1). This is consistent with the strong $\sim 7-8 \mathrm{MHz}$ signals seen on the FFT's in Figures 3.18 and 3.19 .

We also note that the Mike Kang's circuit model of the column exhibited the rise timeexcited $7.8 \mathrm{MHz}$ oscillations on the upper three sections but not on the lower sections (Figures 3.12 and 3.13), again in agreement with the $7.8 \mathrm{MHz}$ EM mode pattern.

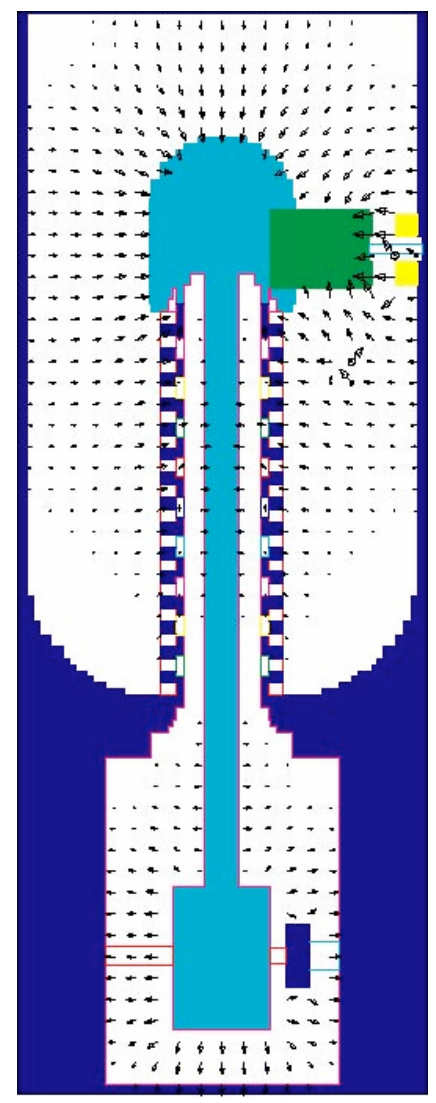

E-field map

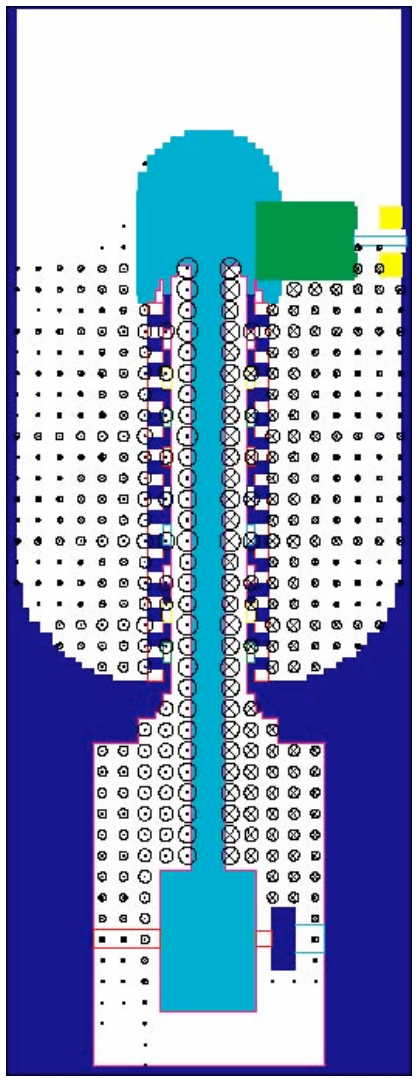

B-field map

Figure 3.22 Lowest mode, at $\sim 8 \mathrm{MHz}$. Arrows are proportional to log of magnitude.

The field map for the second mode predicted by the model, at around $16 \mathrm{MHz}$, is shown in Figure 3.23. In this case the electric field around the bottom of the column is significant, so we would expect this mode to couple to the column CVR. This is a plausible explanation for the strong $18 \mathrm{MHz}$ signals seen on the column CVR when the crowbar is fired. (see Figure 3.17). In addition, B-Dot \# 1 is located in a region where the RF magnetic field of this mode is again appreciable, so it is no surprise that the mode also appears strongly on this diagnostic.

For completeness, the full spectrum of longitudinal modes predicted by this EM model are summarized in Table 3.2. Inferences of a connection between these predictions and 
the higher frequency peaks in the data can clearly be made, but detailed field maps and data from a number of diagnostic locations would be required to really establish these connections.

\begin{tabular}{|l|l|l|l|l|l|l|}
\hline $8 \mathrm{MHz}$ & $16 \mathrm{MHz}$ & $33 \mathrm{MHz}$ & $47 \mathrm{MHz}$ & $56 \mathrm{MHz}$ & $63 \mathrm{MHz}$ & $69 \mathrm{MHz}$ \\
\hline
\end{tabular}

Table 3.2

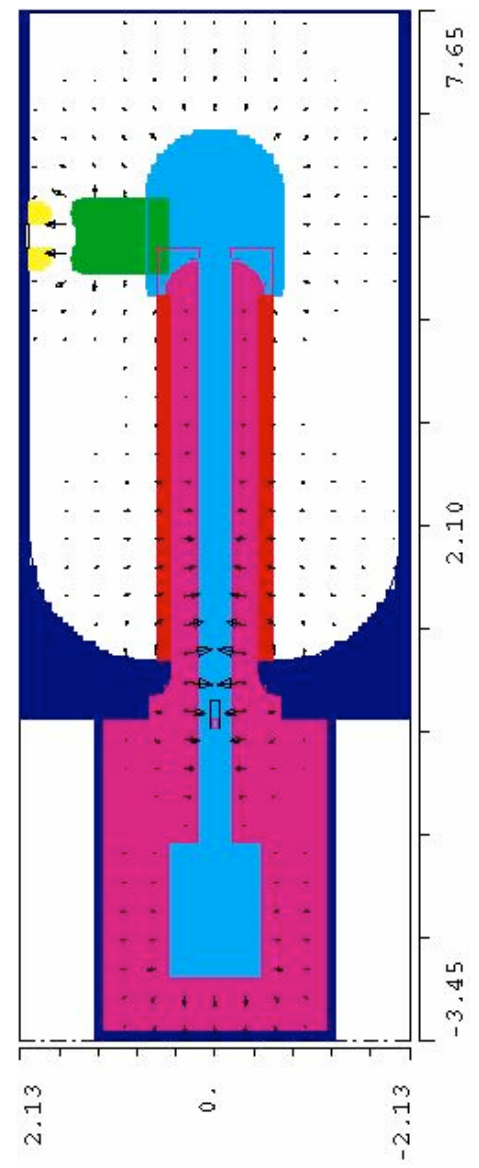

E-field map

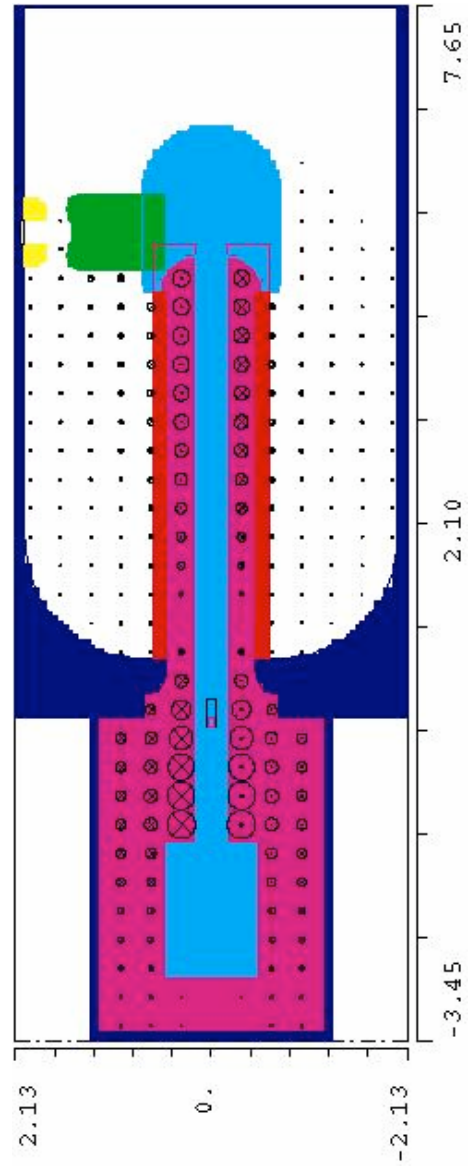

B-field map

Figure 3.23 Second mode at $\sim 16 \mathrm{MHz}$. Arrows are proportional to log of magnitude.

\subsubsection{Modifications in the Crowbar Circuitry}

The excitation of a broad spectrum of high frequency modes by the crowbar, extending up to $50 \mathrm{MHz}$ and even beyond, is clearly a consequence of the rapid voltage collapse of 
the Marx dome from the closure of the crowbar switch. Since the amplitude of some of these modes appears to be in the uncomfortably high range (e.g., the $6.7 \mathrm{MHz}$ on the source dome, the $18 \mathrm{MHz}$ on the column CVR), it makes sense to try to find ways to slow down the Marx dome voltage collapse rate.

Mike Kang has done a preliminary study of the effect of varying the crowbar $\mathrm{R}$ and $\mathrm{L}$ on the 6.7 MHz mode with his circuit model [4]. He has found an "optimum" set of values to eliminate the voltage reversal on the source dome without significantly prolonging the voltage collapse timescale, for example. The predictions of his model using the "nominal" crowbar R and L values are shown in Figure 3.24.

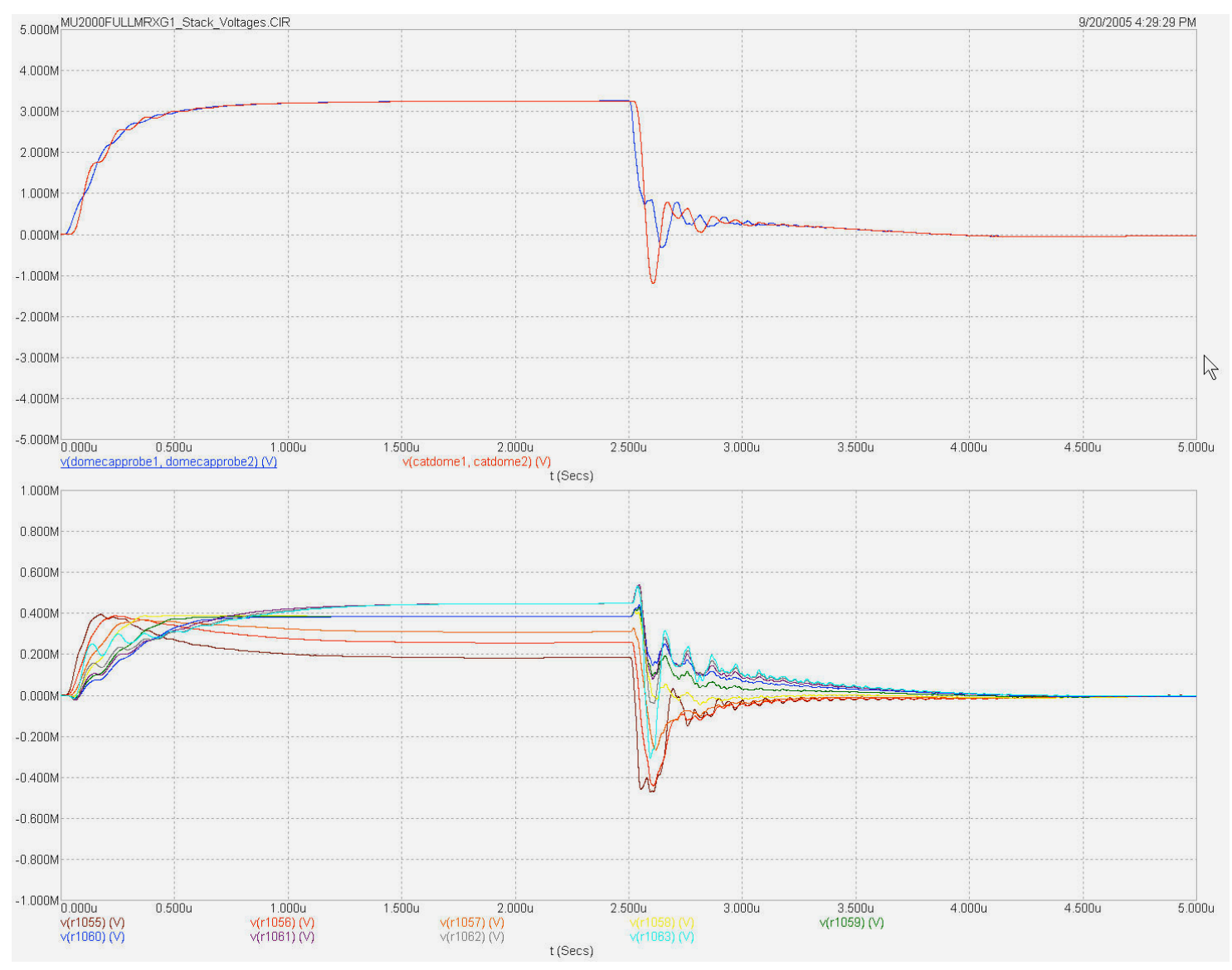

Figure 3.24 Nominal crowbar parameters: $\mathrm{L}=1.3 \mathrm{uH}, \mathrm{R}=30 \mathrm{Ohms}$. Source dome (red) and Marx dome (blue) voltages in the top Figure. The nine column section voltages are shown in the bottom figure, with the lowest (brown) curve the bottom section, etc.

In this $3.2 \mathrm{MeV}$ simulation, the source dome voltage reversal is more than a megavolt. The column model includes the MOV's, which all turn on at crowbar time. The MOV's in fact reduce the Stack 1 reversal from $\sim 600 \mathrm{kV}$ to $\sim 450 \mathrm{kV}$ with small increases across the other stacks. 
The predictions of his model using the "optimum" set of crowbar R and L values are shown in Figure 3.25. The reversal of the source dome voltage has been eliminated with this choice of crowbar parameters. Reversal of the voltages across several of the individual column sections still occurs, however. In fact, reversal of the voltage across the bottom stack occurs at about the same level ( $\sim 10 \%$ less), although the next stack has its reversal reduced by $\sim 1 / 3$. Other choices of the crowbar parameters can reduce these column section reversals even more, but complete avoidance of reversal may prove to be difficult to achieve.
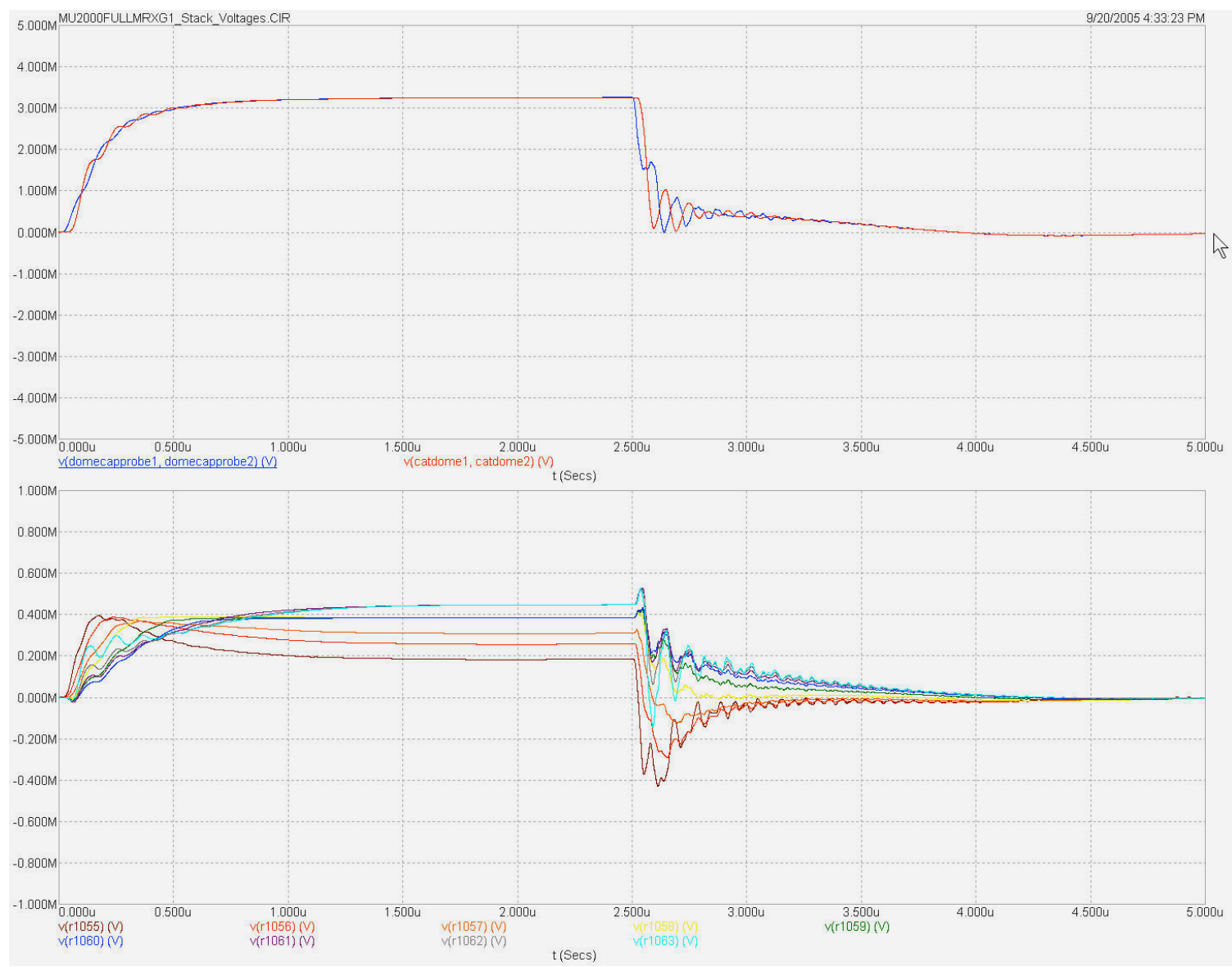

Figure 3.25 Optimum crowbar parameters: $\mathrm{L}=2.5 \mathrm{uH}, \mathrm{R}=45 \mathrm{Ohms}$. Source dome (red) and Marx dome (blue) voltages in the top Figure. The nine column section voltages are shown in the bottom figure, with the lowest (brown) curve the bottom section, etc. 


\section{Design of the Ferrite Damper and Small Scale Circuit Tests}

\subsection{Introduction}

The observation of large amplitude crowbar-induced oscillations over a broad frequency spectrum in the initial injector operation was the main stimulus originally for the ferrite damper concept described in this section. Following the initial preliminary development of the concept in early 2004, very little attention was devoted to it following a Project decision to curtail further use of the crowbar [6,7]. Interest was renewed around June 2005 when the Injector Working Group was formed, with the objective now focused more heavily on utilizing it to damp the rise time induced $7.8 \mathrm{MHz}$ oscillations for the upcoming series of beam experiments.

An "Interim Design Review" of the ferrite damper concept was held at LANL on July 13, 2005, shortly after effort on it at LBNL was restarted [8]. An update was presented at a videoconference on September 8, 2005 to key members of the LANL Project staff and the Interim Design Review chairman (Ken Prestwich). At this videoconference, the data base supporting the final choice of ferrite material was presented.

In this section, we give a brief summary of the design concepts and the small scale validation tests. Mechanical details are covered in Section 5, and the performance is discussed in Section 6.

\subsection{Design Concepts}

Ferrite is used as an electromagnetic wave absorber in many applications, and it is a very logical choice to consider for damping the unwanted RF modes in the present application. Figure 4.1 shows a plot of the small amplitude complex permeability of a typical ferrite material as a function of frequency. Above a critical frequency (the "spin resonance"), the imaginary part of the small amplitude complex permeability becomes comparable to or even greater than the real part, eventually falling off with frequency as $\sim 1 / \mathrm{f}$. This is the high absorption frequency band of this material and the ferrite material properties can be tailored to shift its onset. In NiZn ferrites for example the spin resonance frequency is increased as the relative concentration of $\mathrm{Ni}$ is increased (e.g., like from a few $\mathrm{MHz}$ to 100 's of MHz). 


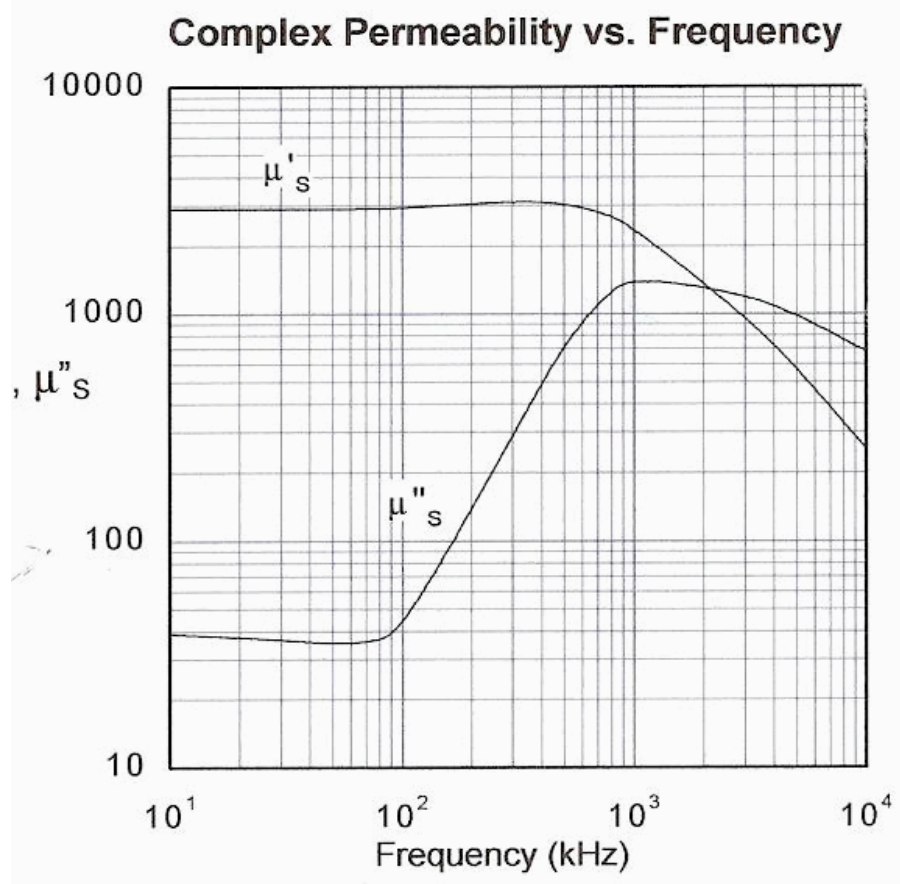

Figure 4.1 Small amplitude complex permeability vs. frequency for a typical NiZn ferrite.

Ferrite tiles are used in the DARHT-2 accelerator cells to damp the transverse (BBU) modes, so their use is not new to the Project [9]. The mode Q's in the cells were reduced from around 30 to $<6$ by the addition of the ferrite tiles, and the initial beam experiments have verified that growth of transverse BBU modes in the machine is minimal.

Key features of the cell ferrite insert design include:

- A tile thickness chosen to minimize RF wave reflections over the $200-500 \mathrm{MHz}$ band $(5.5 \mathrm{~mm})$

- Azimuthal gaps between the tiles (1/8") to avoid saturation of the ferrite by the azimuthal magnetic field of a 2 - 4 KA beam.

Ferrite inserts in the injector appear to be a viable option for damping both the rise time excited 7.8 MHz oscillations, as well as the full spectrum of crowbar-excited modes. Key differences compared to the cell inserts include the following:

- The frequency spectrum is much lower: different ferrite materials may be needed, the required ferrite volume is much larger, and at $6-8 \mathrm{MHz}$ the ferrite toroid acts more like a "lumped element resistor" than a wave absorber.

- The amplitude of the RF magnetic fields created by the oscillating stalk current is large enough to drive the ferrite toroid into saturation even on the rise time (azimuthal gaps are used to limit these nonlinearities). 
Several possible locations for ferrite inserts were considered. The one that was chosen is shown in Figure 4.2. Ferrite slabs are placed against the Marx Tank Skirt shown in the Figure, to form a toroid. The mechanical details of this installation are described in more detail in Section 5. The attractive aspects of this choice include its proximity to the peak of the stalk current for the $7.8 \mathrm{MHz}$ oscillations, a location in the oil and in a region of relatively small electric field stress, and a (relatively) straightforward assembly process without a need to remove the stalk or move the Marx dome out of the way.

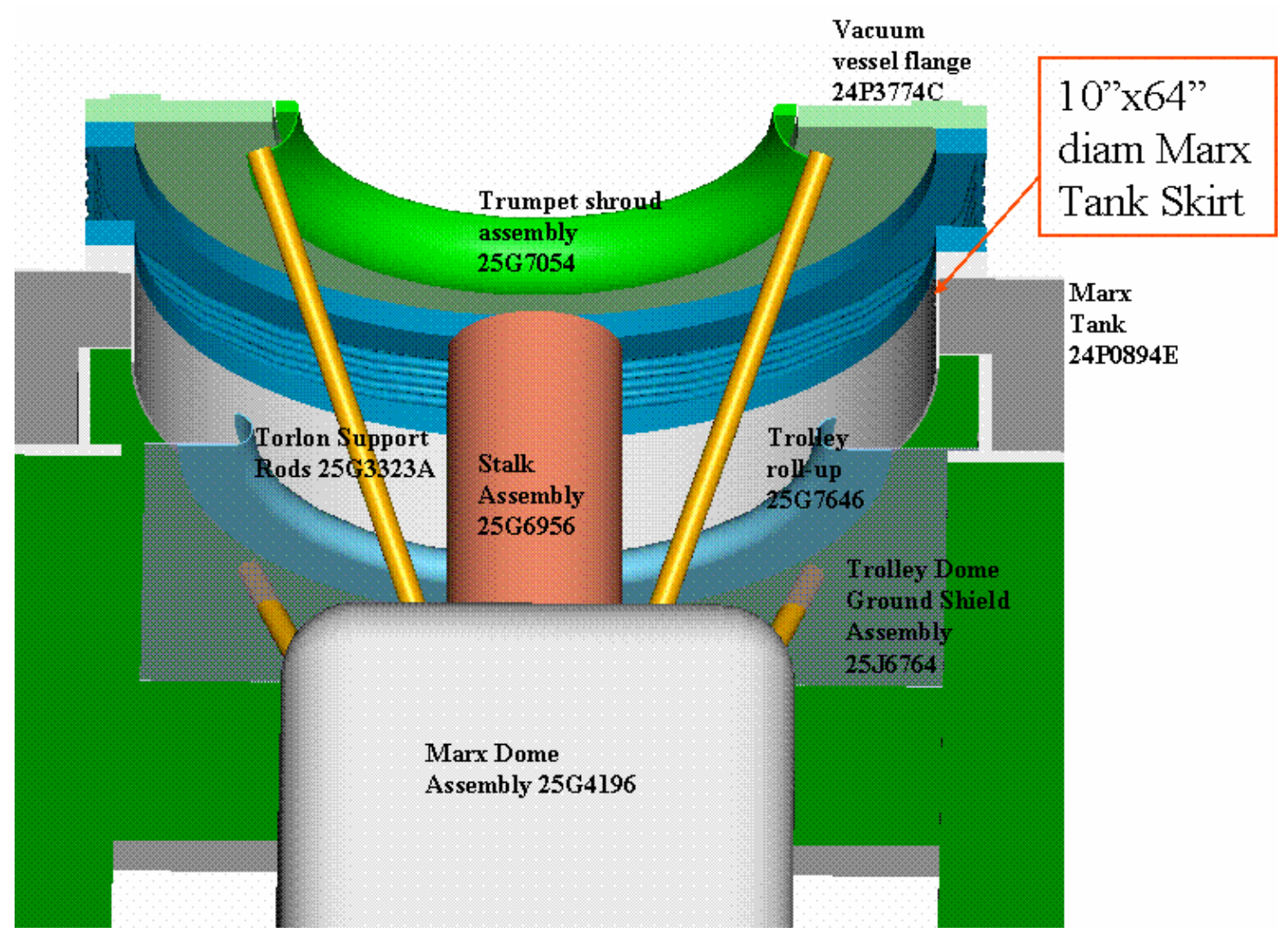

Figure 4.2 Ferrite toroid placement.

A lumped element circuit model of the ferrite toroid should suffice to estimate the damping of 7-8 $\mathrm{MHz}$ oscillations. The basic assumptions are a wavelength and "skin depth" in the ferrite much larger than the toroid radial thickness. For example, in the small amplitude limit when $\operatorname{Im}(\mathrm{mu})>>\operatorname{Re}(\mathrm{mu})$, the RF ferrite impedance (without any gaps in the toroid) is purely resistive and equal to

$$
R=\frac{\sigma_{m} A}{2 \pi r} \quad \text { ohms }
$$


Here $\mathrm{A}$ is the cross sectional area of the torus (radial thickness times axial height), $\mathrm{r}$ is its mean radius, and the magnetic conductivity is defined as

$$
\sigma_{m}=\omega \mu_{0} \operatorname{Im}(\mu)
$$

Typical values for the magnetic conductivity for the ferrite materials we have considered are in the range $\sim 2-5 \times 10^{4} \mathrm{ohms} / \mathrm{m}$. Using $\sigma_{m}=3 \times 10^{4} \Omega / \mathrm{m}$, a ferrite toroid radius a little less than the Marx Tank Skirt radius $(0.8 \mathrm{~m})$, and $A=100 \mathrm{~cm}^{2}$, the ferrite torus (in the small amplitude limit and without any gaps) is equivalent to a resistance of $60 \mathrm{ohms}$ in series with the stalk inductance in the equivalent circuit in Figure 3.4. This series resistance would result in a 0.1 microsecond (e-fold) damping time of the $7.8 \mathrm{MHz}$ rise time induced oscillations. The actual damping will be less than this, however, because the RF fields are large amplitude, so gaps are needed as we explain below.

It is well known that when a ferrite material is driven into saturation, the (incremental) permeability and losses drop dramatically. To avoid this strong saturation regime and maintain a high resistive component of the ferrite impedance, we must use one or more azimuthal gaps in the ferrite toroid. In a ferrite toroid with a small gap $=\Delta$, the azimuthal B field is constant across the gap. From $\oint H \bullet d s=I$ we have

$$
2 \pi r H+\frac{B}{\mu_{0}} \Delta=I
$$

with $\mathrm{H}$ the azimuthal magnetic field inside the ferrite material. The operating point on the typical dynamic B-H curve illustrated in Figure 4.3 is therefore determined by its intersection with the "negative gap line" defined by Eq. 4.3. At a given instantaneous current I, the B field can be kept out of saturation by choosing a large enough gap width, since the slope of the "negative gap line" is $-\mu_{0} 2 \pi r / \Delta$. It is clear that the gap width should be large enough to avoid saturation with the "steady" stalk current (in the pulse flat top) intersecting the "slow" B-H loop. However the "AC" component of the stalk current during the rise time of the pulse is several times greater than this "steady" current (see Figure 3.2), so it is not obvious what current one should use to estimate the required gap width. Fortunately, the response of a ferrite toroid with azimuthal gaps can be accurately represented by the scaled circuit discussed in Ref. [7] and in Section 4.3 in this report. This scaled circuit has been our main tool for optimizing the damping vs. gap spacing. Note that with a gap big enough to avoid saturation, the ferrite is effectively "demagnetized" after the pulse is over (returns to $I=0$ ), as indicated in Figure 4.3. There are no serious questions, therefore, about the magnetization state of the ferrite at the beginning of the next pulse. In addition, with a gap in the ferrite toroid any reset that might be considered to increase the initial flux swing would have to be active (bias current on when the pulse is fired). 


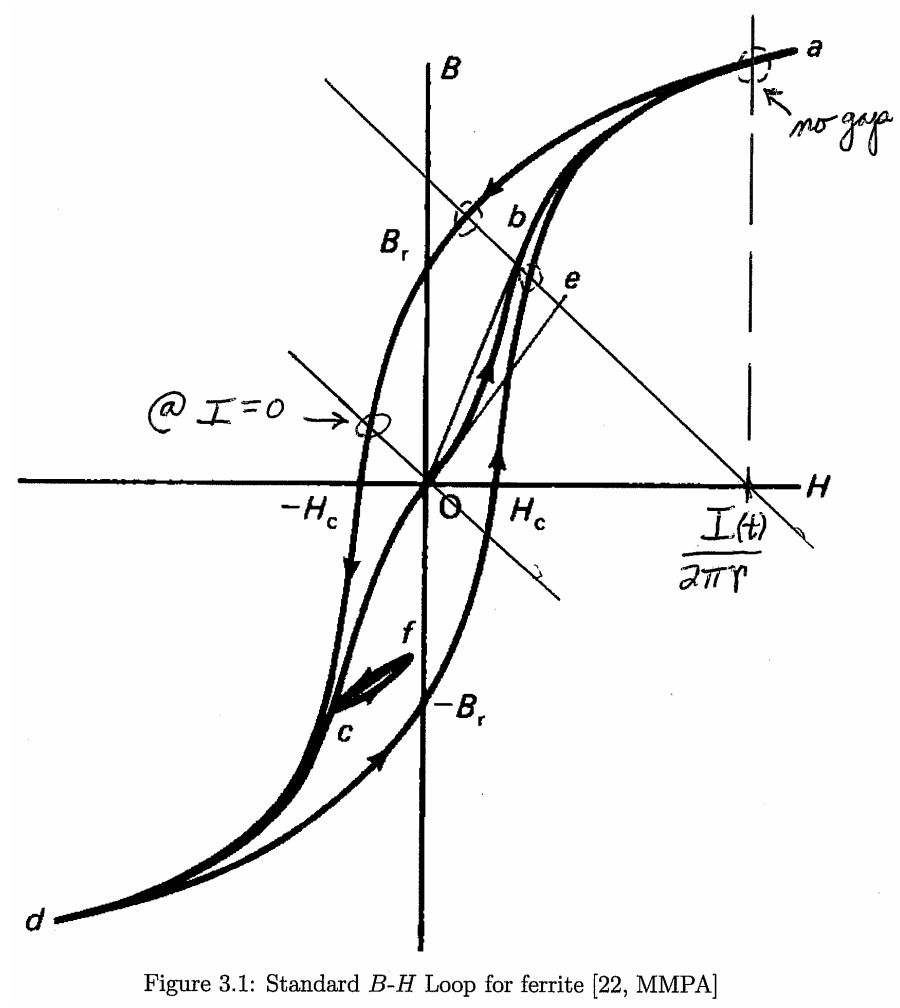

Figure 4.3 The "negative gap line" determines the operating point on the dynamic B-H curve.

A number of candidate ferrite materials have been considered, and tests on the small scale circuit were carried out for several of the most promising. Initially, MnZn appeared to be a good choice, but it was later eliminated as a candidate because of the so-called "dimensional resonances" that occurs with this material (it has a very high effective dielectric constant). Several NiZn ferrites and gap configurations were then tested on the small scale circuit, and on the basis of these tests CN20 with a 9 mil gap was chosen for its effectiveness and availability. 


\subsection{Small Scale Tests}

The electromagnetic response of the ferrite toroid in the injector application is very complicated (compared to the ferrite tiles used in the accelerator cells, for example). In addition to the dispersive nature of the material (frequency dependence) evident even with small amplitude EM fields, the response of the injector ferrite also involves nonlinearities (saturation) and memory (hysteresis). The small scale hardware circuit model described here is designed to produce the same "trajectories" in B-H space in the small ferrite toroid as in the injector toroid, both in the temporal rate and the amplitude of $\mathrm{B}$ and $\mathrm{H}$. This hardware model is the essential tool we have used to predict the performance of the injector ferrite insert, guiding the selection of the ferrite material and the identification of an optimum gap spacing. The task of developing an accurate computational model would be formidable, and far more costly in dollars and manpower.

The small scale test circuit schematic shown in Figure 4.4 is basically the same as the simple injector circuit model in Figure 3.4, except for a 10x reduction in the impedance.

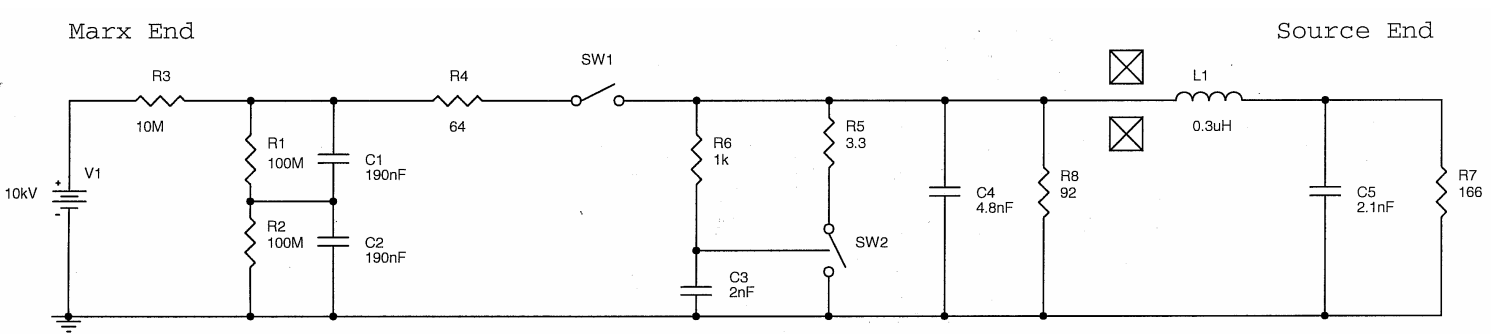

Figure 4.4: Small scale test circuit with simulated beam.

The scaling between the circuit stalk current and the injector stalk current should maintain the same $\mathrm{H}$ field in the ferrite toroid, so the ratio of the stalk current to the ferrite core radius should be a constant. With a mean radius of the sample core of about $1.125 \mathrm{~cm}$, the small scale circuit current is lower by a factor of 68 compared to the injector stalk current. The impedance was arbitrarily chosen to be lower by a factor of 10. The scaled voltage in the circuit is then lower by a factor of 680 . The ratio of voltage to core area should be an invariant to get the same B, so the area of the sample core is lower by a factor of 680 compared to the area of the equivalent ferrite toroid in the injector. The gap in the sample core scales to the (total of all the) gap(s) in the injector toroid by maintaining the same ratio of gap width to core circumference using the mean radius. The gap width in the sample core is therefore smaller by a factor of 68 compared to the equivalent total gap width in the injector ferrite toroid. A summary of the circuit parameters and their correspondence with the injector parameters are given in Table 4.1. 


\begin{tabular}{|c|c|c|c|}
\hline Parameter & Injector & Circuit & $\begin{array}{c}\text { Factor (circuit to } \\
\text { injector) }\end{array}$ \\
\hline $\begin{array}{c}\text { Source Dome } \\
\text { Voltage }\end{array}$ & $3.4 \mathrm{MV}$ & $5.05 \mathrm{kV}$ & 680 \\
\hline Stalk Current & $2.1 \mathrm{kA}$ & $31 \mathrm{~A}$ & 68 \\
\hline Ferrite Radius & $77 \mathrm{~cm}$ & $1.125 \mathrm{~cm}$ & 68 \\
\hline Ferrite Area & $170 \mathrm{~cm}^{\wedge} 2$ & $0.25 \mathrm{~cm}^{\wedge} 2$ & 680 \\
\hline $\begin{array}{c}\text { Ferrite Gap } \\
\text { Width }\end{array}$ & $1.56 \mathrm{~cm}$ & $0.0229 \mathrm{~cm}$ & 68 \\
\hline
\end{tabular}

Table 4.1 Scaling from the small scale circuit to the injector.

A $166 \mathrm{ohm}$ resistor loading the "source dome C" was chosen to represent the "with beam" condition (a scaled flat top stalk current of $2.1 \mathrm{kA}$ ) while a resistor of $629 \mathrm{ohms}$ was chosen for the "no beam" condition to have the stalk current relax to the correct steady column current. Note that with a single resistor across the source dome $\mathrm{C}$ the circuit does not recreate the initial capacitive current "surge" from the charging of the distributed column to stalk capacitance discussed in Section 3.4. The circuit also does not recreate the higher frequency ringing modes that are excited by the crowbar.

Several different ferrite materials were considered and tested before choosing CN20. The damping using the "with beam" configuration with a CN20 core was then tested with a variety of gap widths. On the basis of these tests, the 9 mil gap was considered to be the best choice. The damping is actually relatively insensitive to gap width over a range from around 6 mils to 12 mils, so precise control of the gaps in the actual installation should not be required.

\subsubsection{The "No Ferrite Damper" Baseline}

The "source dome voltage" and "stalk current" with the "no beam" configuration in the small scale circuit are shown in Figure 4.5. The oscillation frequency in the rise time is $7.8 \mathrm{MHz}$, and the e-fold damping time constant of the rise time oscillations is about 0.74 microseconds (compared to 0.8 microseconds in the real injector). The e-fold rise time of the source and Marx dome voltages is about 0.2 microseconds (compared to 0.23 microseconds in the injector). 
Similar data using the "with beam" configuration show an e-fold voltage rise time of 0.16 microseconds (compared to 0.19 microseconds in the real injector), and an e-fold damping time constant of the $7.8 \mathrm{MHz}$ oscillations of 0.45 microseconds (compared to 0.5 microseconds in the injector).

The comparison of these "no ferrite" circuit time constants with the injector observations discussed in Section 3.3 is summarized in Table 4.2.

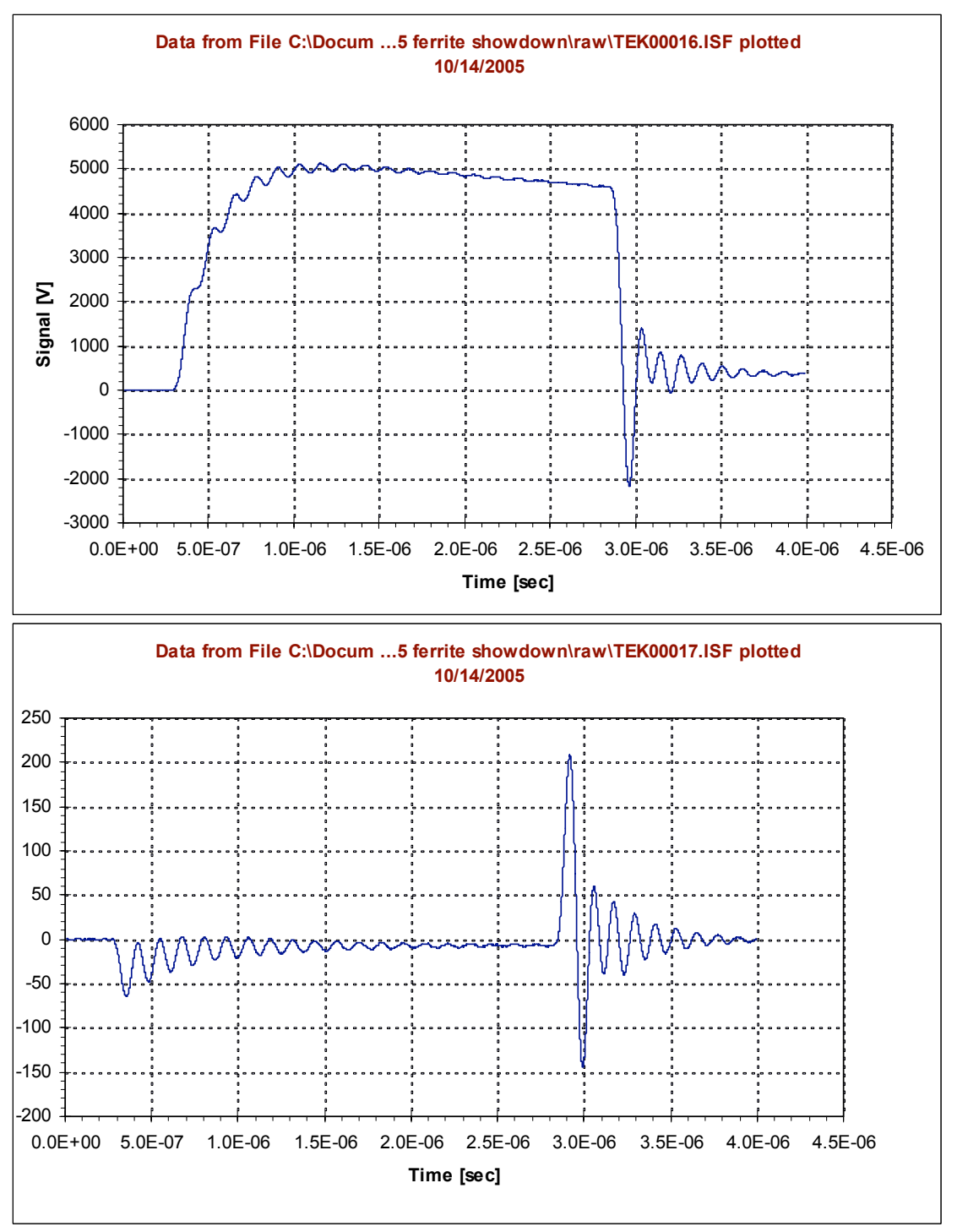

Figure 4.5 Source dome voltage (upper) and stalk current (lower, inverted scale, in amps) for the "no beam" configuration without ferrite. 


\begin{tabular}{|c|l|l|}
\hline Time constant (rise time) & Injector & Circuit \\
\hline No beam - voltage rise time $\tau_{r}$ & $0.23 \mu \mathrm{sec}$ & $0.2 \mu \mathrm{sec}$ \\
\hline - oscillation damping $\tau_{d}$ & $0.8 \mu \mathrm{sec}$ & $0.74 \mu \mathrm{sec}$ \\
\hline With beam - voltage rise time $\tau_{r}$ & $0.19 \mu \mathrm{sec}$ & $0.16 \mu \mathrm{sec}$ \\
\hline - oscillation damping $\tau_{d}$ & $\begin{array}{l}0.5 \mu \mathrm{sec}(1.2 \mathrm{kA} \\
\text { beam) }\end{array}$ & $0.45 \mu \mathrm{sec}$ \\
\hline
\end{tabular}

Table 4.2 Comparison of "no ferrite" circuit time constants with the injector observations.

The scaled circuit therefore does a reasonable job of replicating the waveforms of the real injector, including the excitation level of the $7.8 \mathrm{MHz}$ oscillations on the rise time. This is not surprising considering the good fit between the injector data and the simple circuit predictions. The simulated crowbar in the test circuit (SW2 - a self triggered spark with an R-C network to control the delay) also created waveforms very similar to the actual injector.

\subsubsection{Ferrite With a 9 mil Gap, No Beam Configuration}

The source dome voltage on an expanded vertical scale is shown in Figure 4.6 to illustrate the damping of the rise time oscillations by the ferrite toroid. The case shown is a ferrite toroid with a 9 mil gap and the "no beam" configuration. 


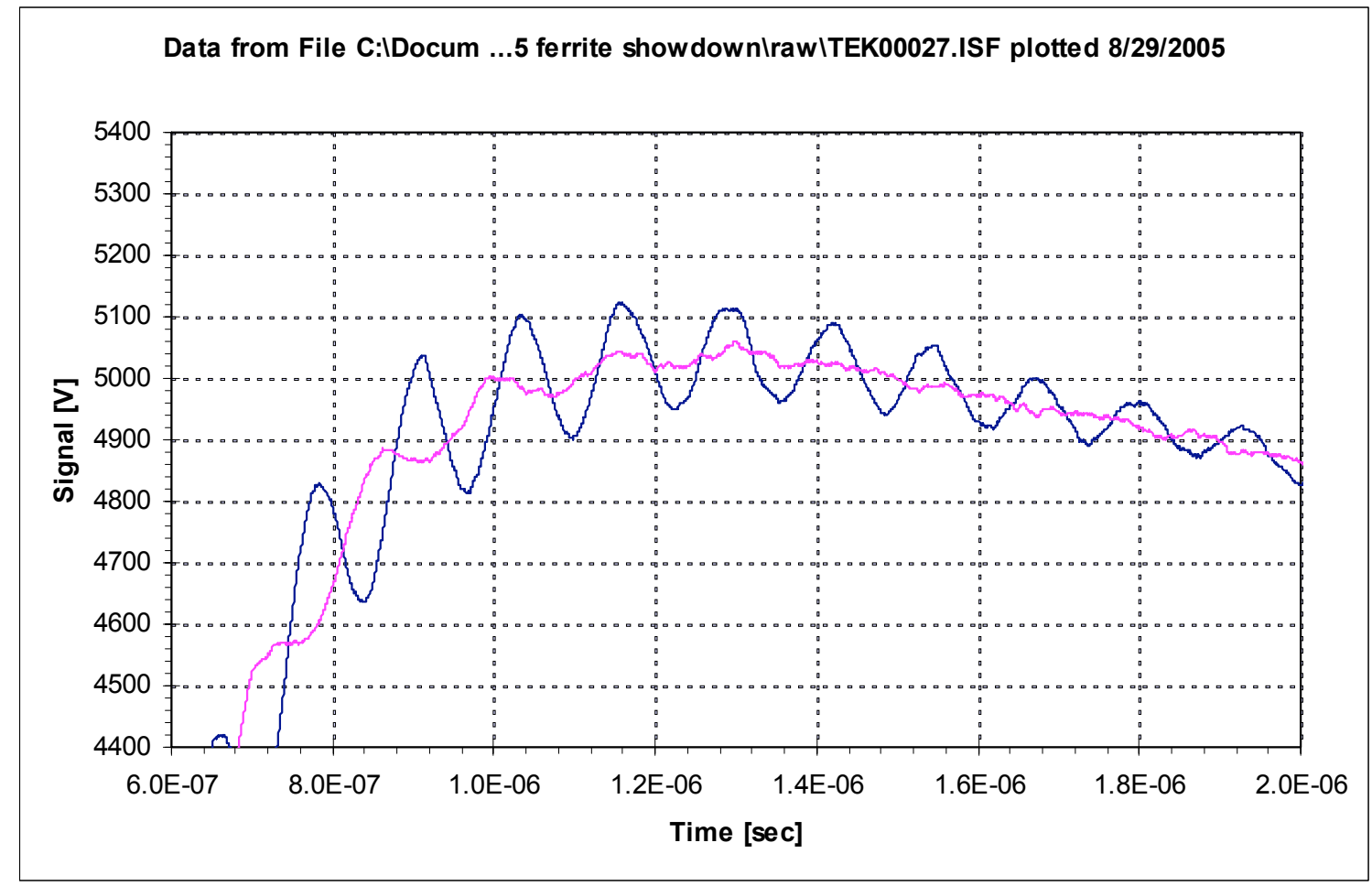

Figure 4.6: Damping effect of ferrite on the source dome voltage oscillations without beam. Blue trace has no ferrite. Magenta trace has a CN20 ferrite toroid with a 9 mil gap.

The ferrite voltage and stalk current at the front part of the pulse for this case are shown in Figure 4.7. 


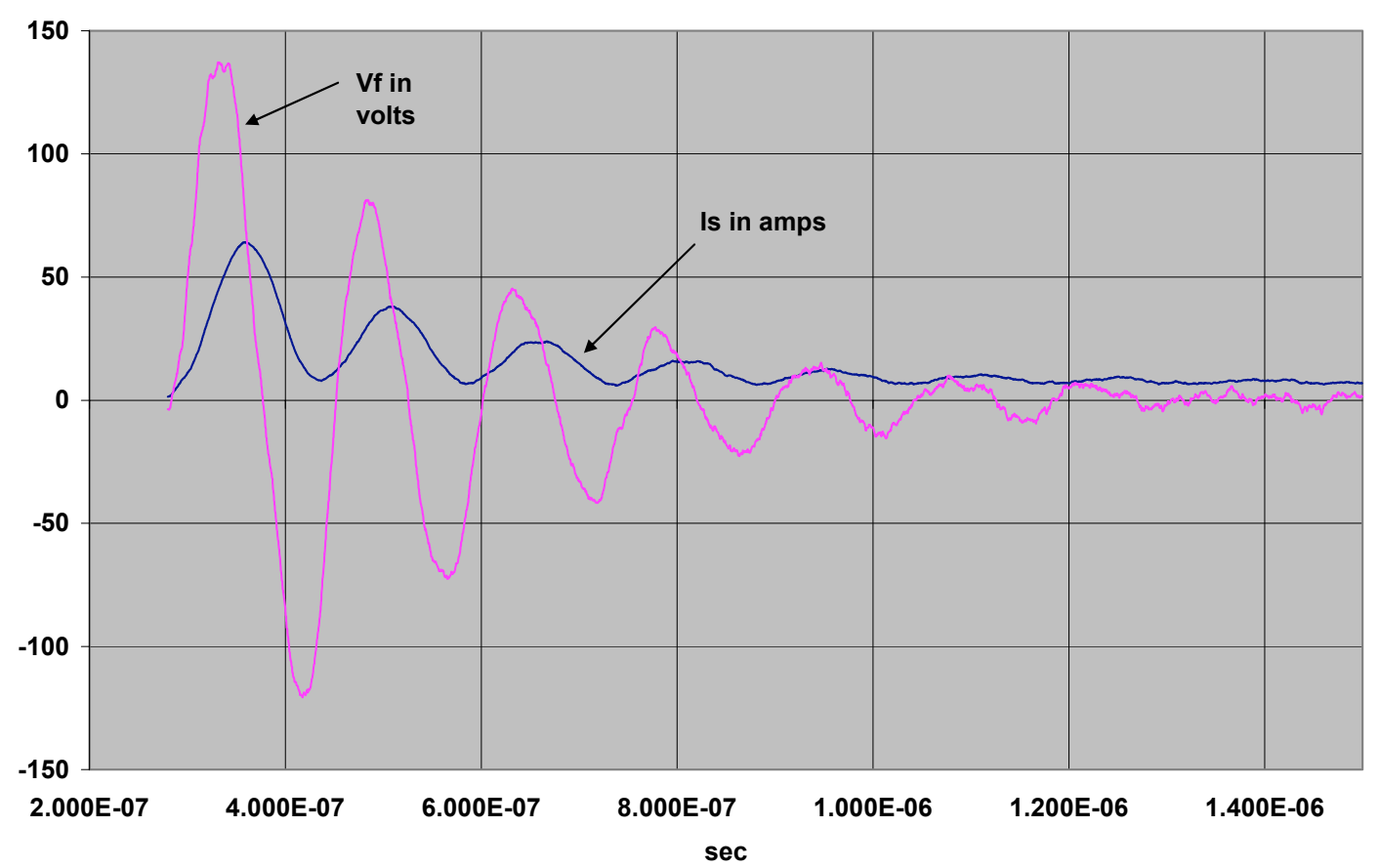

Figure 4.7 Ferrite voltage and stalk current for the 9 mil gap case without beam.

With the ferrite toroid inserted, the frequency of the rise time oscillations is shifted down to around $6.7 \mathrm{MHz}$ because of the added inductance of the ferrite. We do not expect the damping of the oscillations to follow a precise exponential law since the ferrite impedance is nonlinear. However the present case is close to an exponential, with a time constant of around $\tau_{d} \approx 0.24 \mu \mathrm{sec}$, a rate about 3 times faster than without the ferrite.

The peak value of the ferrite voltage is about 130 volts. This translates into a peak voltage of about $90 \mathrm{kV}$ with a ferrite toroid in the injector using the scaling in Table 4.1.

It is instructive to integrate the ferrite voltage, and divide by the toroid area, in order to determine delta B and thereby obtain the "trajectory" of the oscillations and the losses per cycle in B-I space. This is shown in Figure 4.8 for the 4.9 mil ferrite gap case, where we assume that B starts at zero at the front of the pulse (this should be approximately true because of the air gap). Since the loss per cycle is proportional to the area inside the elliptical shaped trajectories, we see that the losses are substantial on the first 2-3 cycles. Also note that the peak value of B stays well below the saturation flux of around $0.35 \mathrm{~T}$ in this case. 


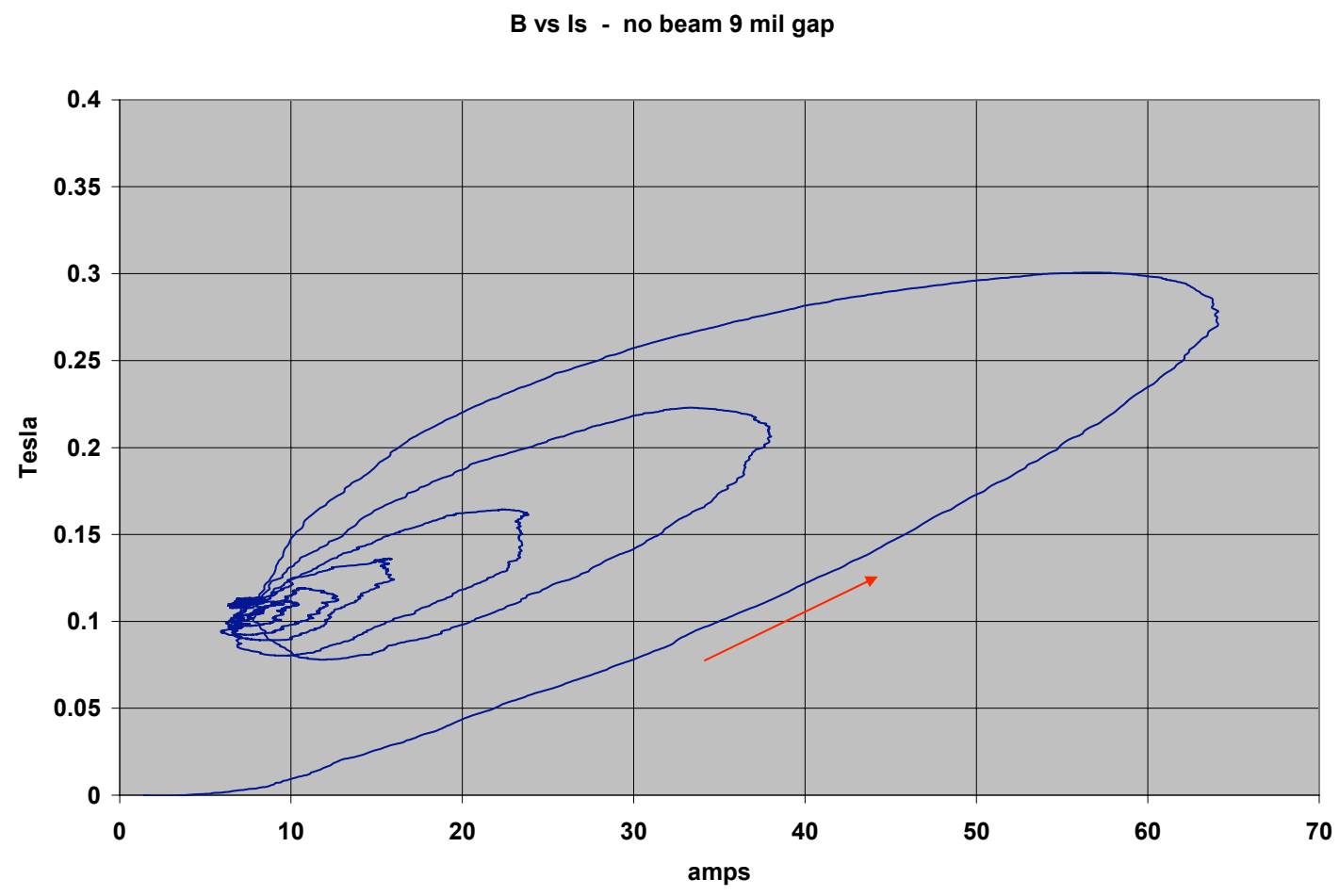

Figure 4.8 B-I trajectory of the CN20 ferrite with no beam and a 9 mil gap.

\subsubsection{Ferrite With a 9 mil Gap in the "With Beam" Configuration}

The data for the "with beam" resistor configuration with a 9 mil gap is shown in Figures $4.9,4.10$, and 4.11. The damping of the oscillations is stronger in this case since the beam load contributes to the damping. From the stalk current oscillations in Figure 4.10 we find the frequency is shifted down to $6.9 \mathrm{MHz}$ and the time constant for the oscillations to decay is now around $0.2 \mu \mathrm{sec}$ (compared to $0.45 \mu \mathrm{sec}$ without the ferrite).

The B-I trajectory shown in Figure 4.11 is similar to the no beam case, except now the current asymptotes to around 32 amps (2.2 kA in the injector) which biases it more towards saturation. The higher first peak of the stalk current also brings the ferrite closer to saturation. 


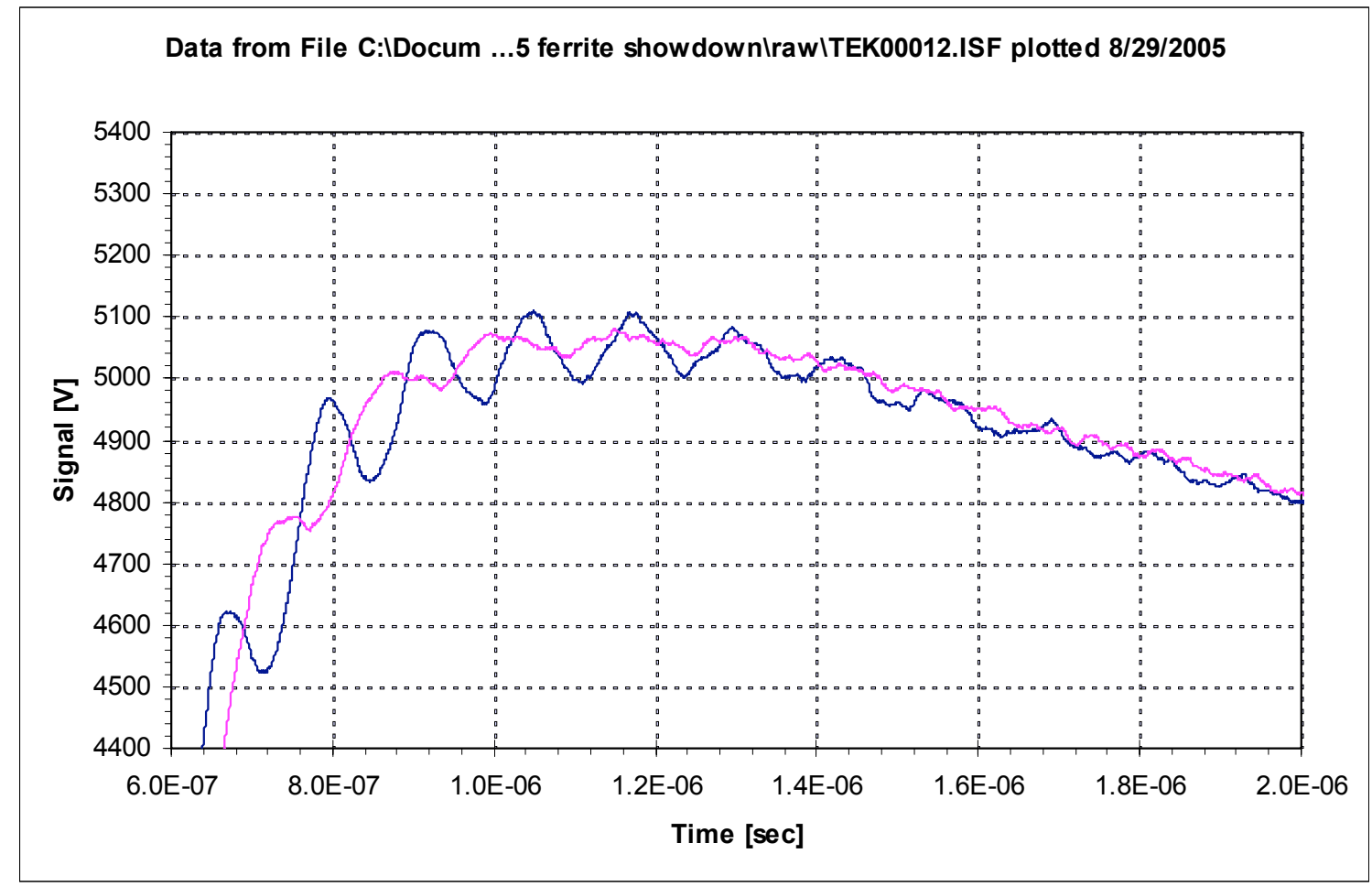

Figure 4.9 Damping effect of ferrite on the source dome voltage oscillations with a simulated beam. Blue trace has no ferrite. Magenta trace has a CN20 ferrite toroid with a 9 mil air gap. 
Vf and Is with beam, 9 mil gap

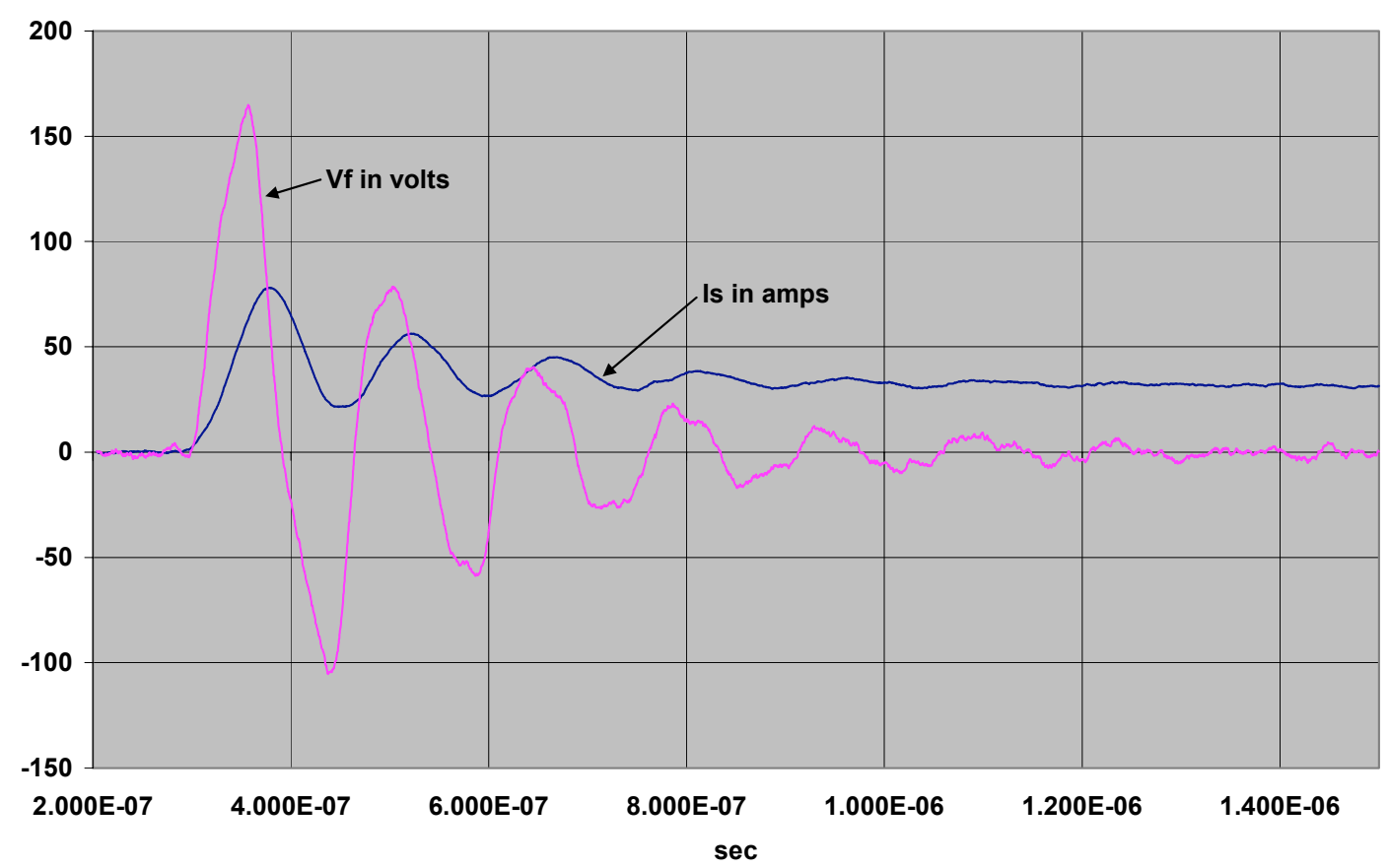

Figure 4.10 Ferrite voltage and stalk current for the 9 mil gap case with a simulated beam.

B vs Is with beam, 9 mil gap

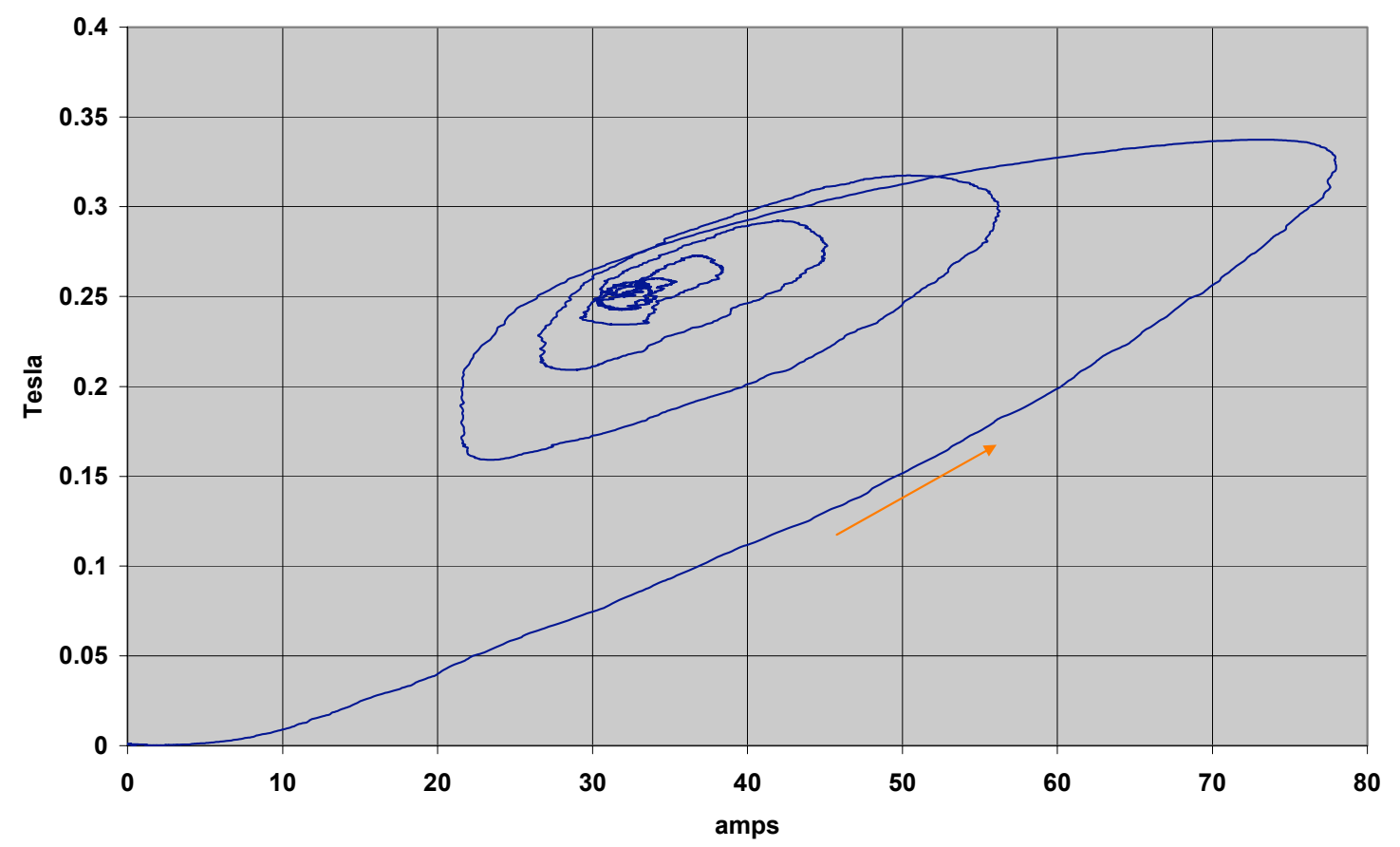

Figure 4.11 B-I trajectory of the CN20 ferrite with simulated beam and a 9 mil gap. 


\subsubsection{Ferrite With No Gap in the "With Beam" Configuration}

To provide a dramatic illustration of the need to avoid saturation, we present the case of no gap in the ferrite (in the "with beam" configuration) in Figures 4.12 and 4.13. In Figure 4.12 the blue trace shows the source dome voltage on an expanded scale with a CN20 ferrite toroid inserted but without any gap. There is very little damping of the oscillations in this case; the decay is about the same as the case without any ferrite.

The B-I trajectories for the no gap case shown in Figure 4.13 provide an explanation. The first peak in the stalk current oscillation drives the ferrite deep into saturation at around $0.35 \mathrm{~T}$. In the subsequent oscillations the incremental "mu" is very small (the slope of delta B vs. I is small) and the loss per cycle (area in the elliptical trajectory) is also very small.

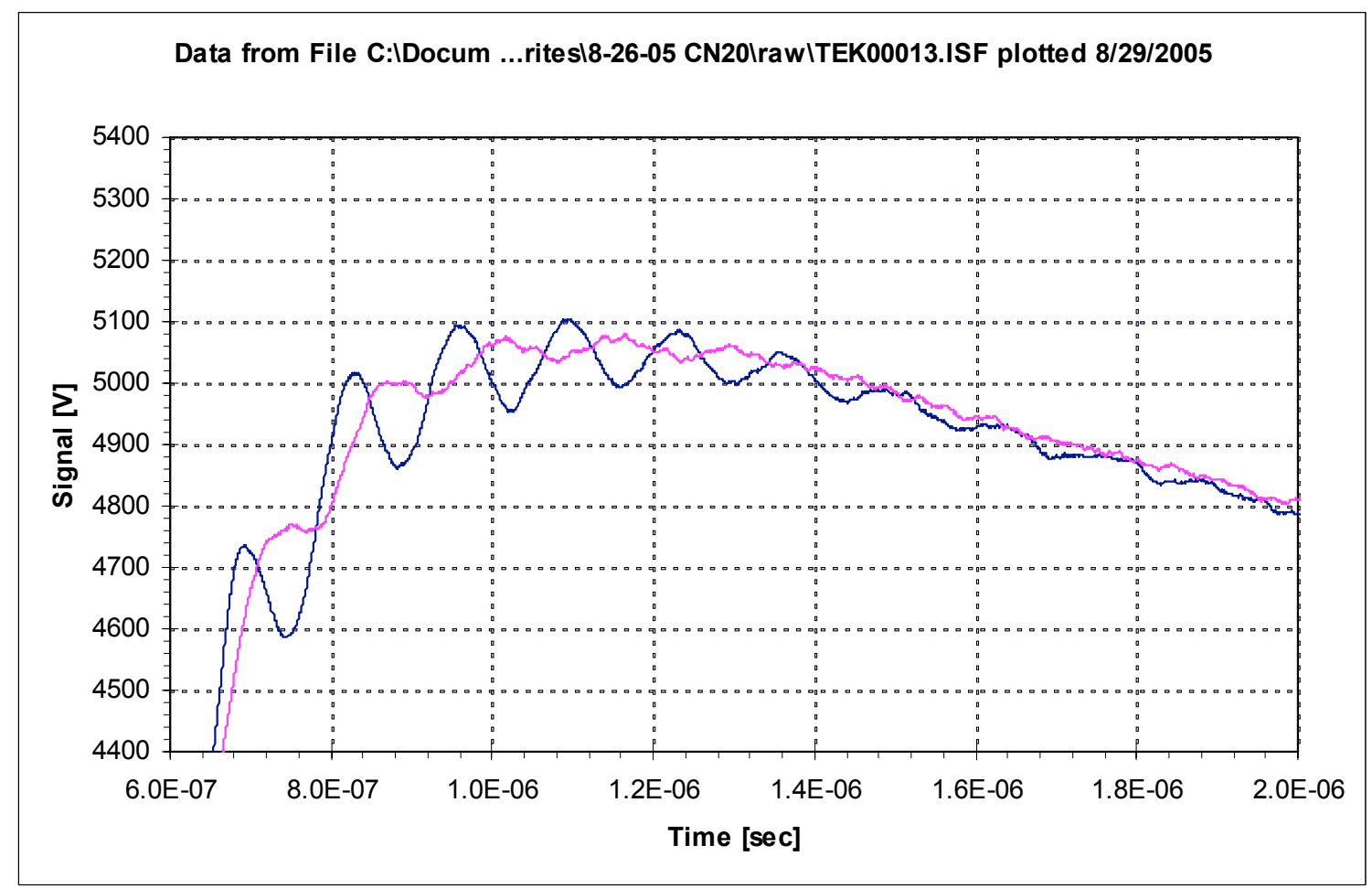

Figure 4.12 Effect of the air gap on source dome voltage damping with beam. Blue trace has CN20 ferrite with no gap. Magenta trace has CN20 ferrite with a 9 mil gap. 


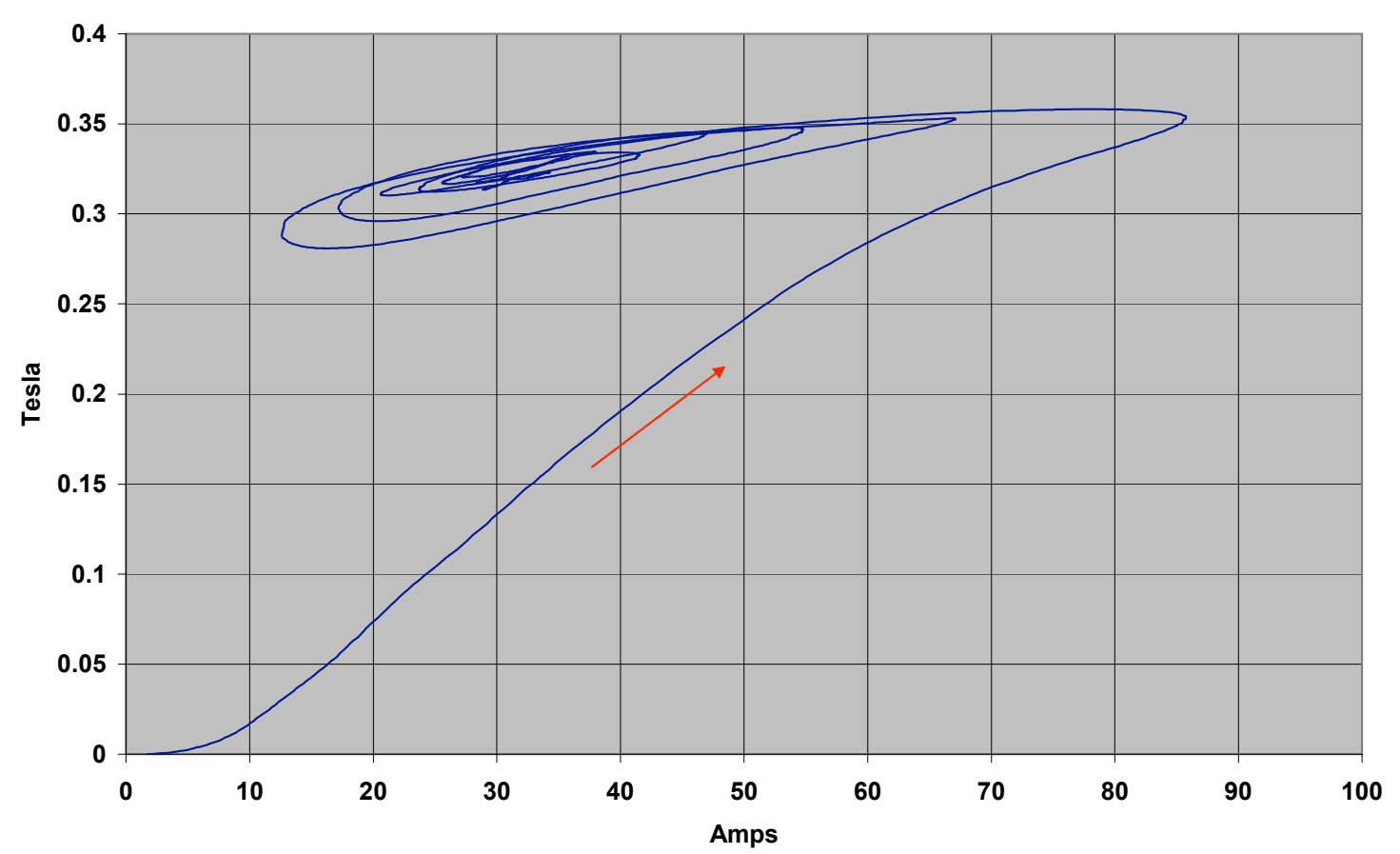

Figure 4.13 B-I trajectory of the CN20 ferrite with simulated beam and without any gap.

\subsection{Conclusions}

On the basis of the small scale circuit testing, a $170 \mathrm{~cm}^{2}$ area toroid insert would damp the rise time induced $7.8 \mathrm{MHz}$ oscillations at a rate $1 / \tau_{d}$, with $\tau_{d} \sim 0.2$ to $0.25 \mu \mathrm{sec}$ (depending on the beam current). It was not clear when the final design choices had to made whether this exponential damping rate would be adequate, since no criteria on the acceptable level of the $7.8 \mathrm{MHz}$ fluctuations had been developed. This acceptable level is determined by the $7.8 \mathrm{MHz}$ transverse beam oscillation amplitudes as well as the injector voltage fluctuation levels, of course.

In the absence of a firm criteria, ferrite tile dimensions were chosen to provide a $210 \mathrm{~cm}^{2}$ area toroid (2.5"x13"), $\sim 25 \%$ greater than the scaled area that was tested. In part, this extra margin was chosen because the actual stalk current waveform in the injector (Figure 3.2) exhibits a higher initial "average" level than the scaled circuit, the difference arising from the "surge" of column charging current in the injector. Unfortunately, reliable stalk current data was not available until very recently so the importance of this difference was not recognized when the scaled circuit tests were carried out. With the additional ferrite toroid area, we also decided to add additional margin to the gap width to ensure the ferrite doesn't get driven too far into saturation.

All of the small scale circuit testing has included the simulated crowbar firing. Additional analysis of this data is warranted, but the major shortfall in assessing the performance of 
the ferrite insert when the crowbar fires is the absence of injector stalk current data as discussed in Section 3. In particular, a major uncertainty is the level of the higher frequency oscillations on the stalk current excited by the crowbar.

The transient voltage stresses on the ferrite insert during the rise time should be modest on the basis of the small scale circuit tests ( $100 \mathrm{kV}$ max over 13" in a 20-30 ns pulse). The choice of a NiZn ferrite material also means we can expect this $\sim 3-4 \mathrm{kV} / \mathrm{cm}$ average stress to be reasonably uniform over the surface of the ferrite tiles.

The crowbar-induced oscillations in the ferrite voltage will be much larger. The ferrite voltage in the small scale circuit with a 9 mil gap ("with beam") is shown in Figure 4.16. The $660 \mathrm{kV}$ voltage in the circuit scales to $\sim 450 \mathrm{kV}$ in the injector, or an average stress around $15 \mathrm{kV} / \mathrm{cm}$. This is still modest for a short pulse. The main uncertainty is the level of the higher frequency modes as already mentioned.

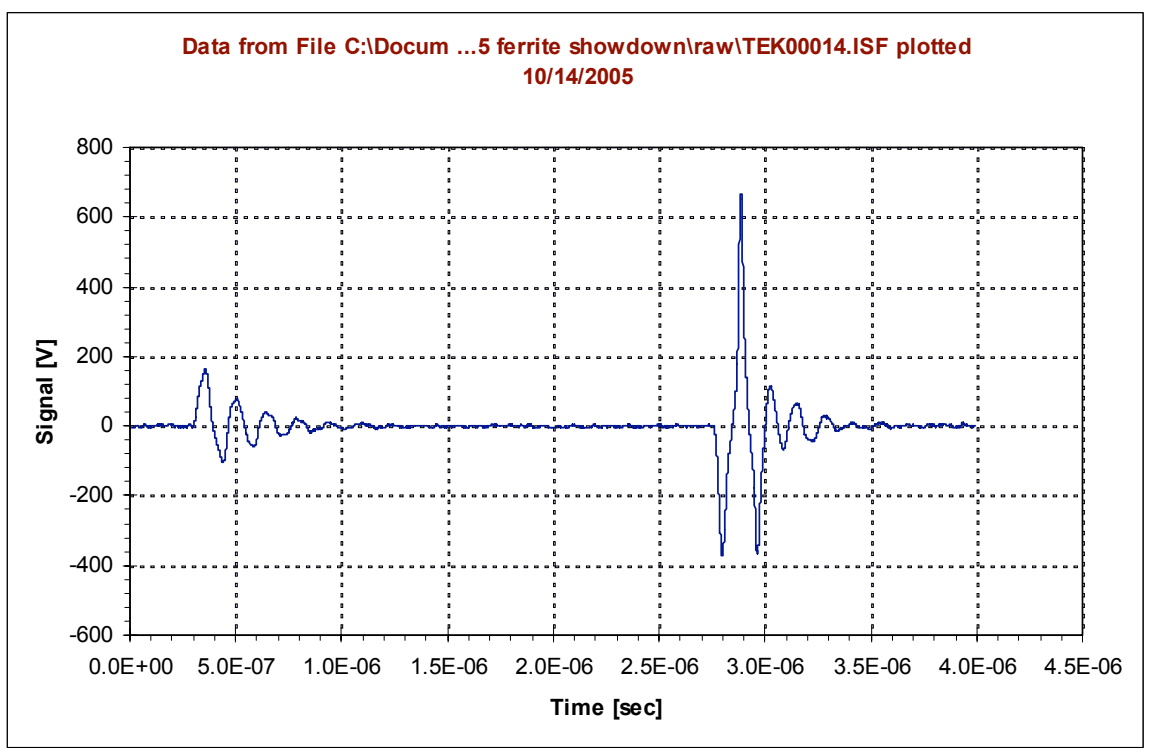

Figure 4.16 Ferrite voltage showing the large crowbar-induced oscillations from the small scale circuit "with beam" and a 9 mil gap. 


\section{Ferrite Damper Mechanical Details and Installation}

\subsection{General Assembly}

The ferrite damper design is implemented using a ring of ferrite tiles resting on a metal shelf that is screwed into the bottom of the Marx skirt (see Figure 5.1). Plastic components are used as spacers between the ferrite tiles and shelf, between the ferrite tiles and Marx skirt, and between the ferrite tiles themselves. A pair of diagnostic loops are embedded in one of the plastic spacers. All the components are in oil.

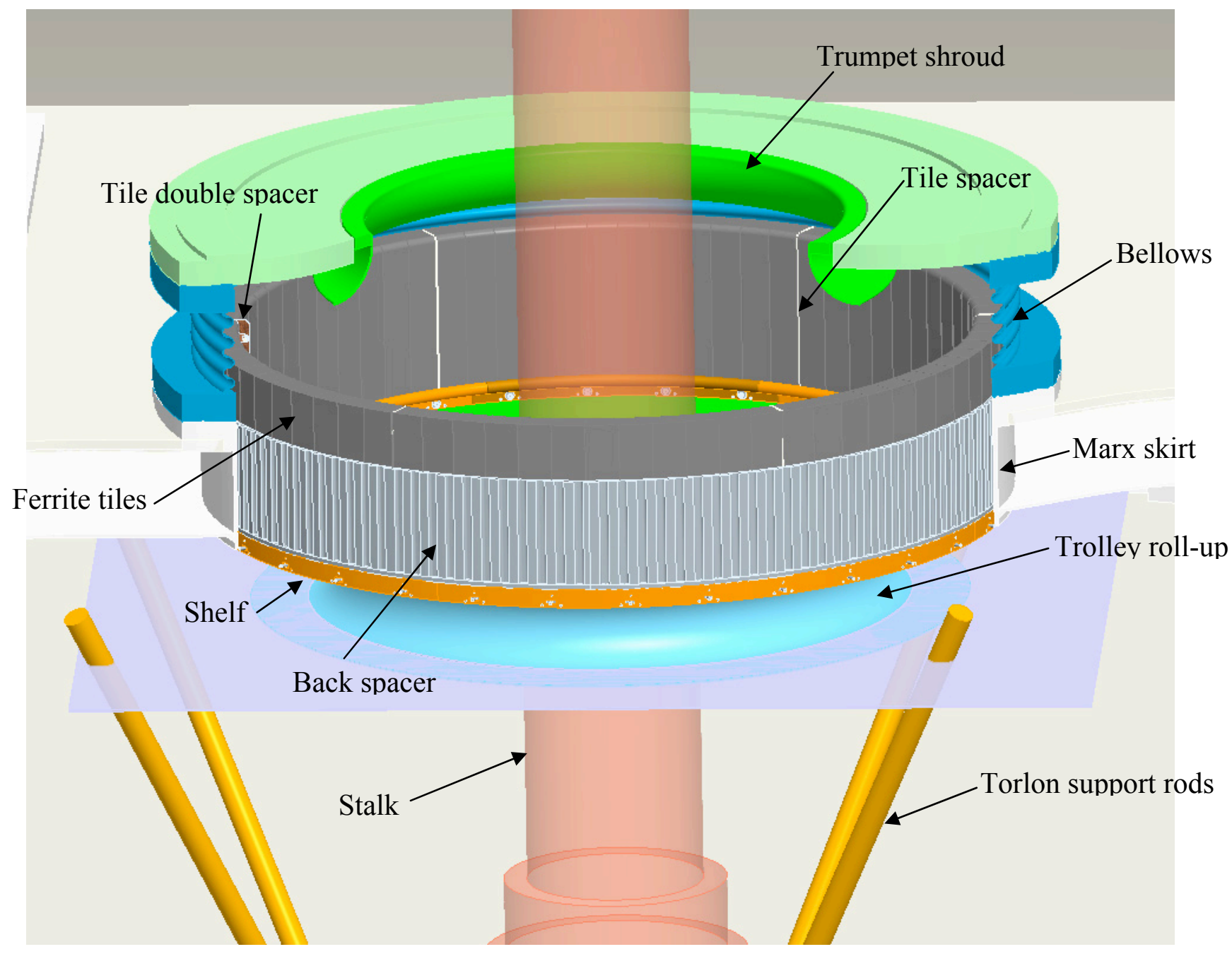

Figure 5.1 Ferrite damper system installed on Marx skirt.

The following drawings document the design:

- LBNL 27D622C: ferrite damper assembly

- LBNL 25J902C: ferrite tile, Ceramic Magnetics, Inc. CN20

- LBNL 27D421C: back spacer, high density polyethylene

- LBNL 27D422B-01: bottom spacer with cutout, high density polyethylene 
- LBNL 27D425D-01: shelf with 3.75 inch wide cutout, aluminum alloy 6061

- LBNL 27D426A: tile spacer, high density polyethylene

- LBNL 27D619B: double spacer wedge, high density polyethylene

- LBNL 27D623B: tile spacer with loop assembly, high density polyethylene

- LBNL 27D655A: back spacer with cable groove, high density polyethylene

- LBNL 27D422B-02: bottom spacer without cutout, high density polyethylene

- LBNL 27D425D-02: shelf with no cutout, aluminum alloy 6061

The ring of ferrite tiles rests on the metal shelf and a pair of plastic spacer wedges is used to apply hoop compression, thereby "locking" the set of tiles and preventing them from moving radially inward. The two spacer wedges are bolted to each other after assembly to prevent relative radial movement between them. No fasteners are used to secure the tiles to the shelf.

\subsubsection{Ferrite Tiles}

Seventy-three of the ferrite tiles are identical, with general dimensions of 12.99 inches tall, 2.69 inches wide, and 2.5 inches deep. The bottom has a 7 degree angle, and the sides have 92.42 degree angles relative to the front and back faces. Refer to drawing LBNL 25 J902C for specific dimensions. The last tile (the $74^{\text {th }}$ tile) was custom cut to size. Each of the standard tiles weigh $16 \mathrm{lbs}$ and the total set weighs slightly less than $1200 \mathrm{lbs}$.

\subsubsection{Gaps Between Tiles (Gap Spacer and Wedge Pieces)}

The tiles are grouped into 4 groups of 12 and 2 groups of 13 . The group of 13 tiles with all full-width tiles is positioned on the east side of the Marx skirt, and the group of 13 tiles with the (last) partial-width tile is positioned on the west side of the Marx skirt (see Figure 5.2). A polyethylene spacer is placed between each group of tiles, and a doublewidth spacer is placed between the groups of tiles on the west side. The double-width spacer is comprised of 2 tapered wedges that serve to azimuthally compress the ring of tiles when the wedges are driven towards each other. The hoop compression created by the double-width spacer serves to lock the tile set and prevent them from moving radially inward. All the tiles and all the single-width tile spacers are keystone shaped (when viewed from the top).

The tile spacers are sized to yield a cumulative azimuthal gap of between 2 and $3 \mathrm{~cm}$. Although the tiles are designed to mate exactly face to face to each other, manufacturing tolerances and slight out-of-roundness in the Marx skirt resulted in small gaps either at the inner edges or outer edges of the tiles. Table 1 lists the final measured gap sizes. The final cumulative gap size after installation is $2.03 \mathrm{~cm}$ at the inner radial location and 2.72 $\mathrm{cm}$ at the outer radial location. 


\subsubsection{Back Spacers}

The tiles are spaced away from the Marx skirt with a set of 3 back spacers made of high density polyethylene. The back spacers are 0.375 inches thick with grooves in them to allow easier bending and oil migration. The back spacers are also only 8.5 inches tall, considerably shorter than the height of the tiles, to avoid interference with the protruding transition from the Marx skirt to the bellows flange. At the west side, one groove is widened to accommodate routing of 16 RG223 cables.

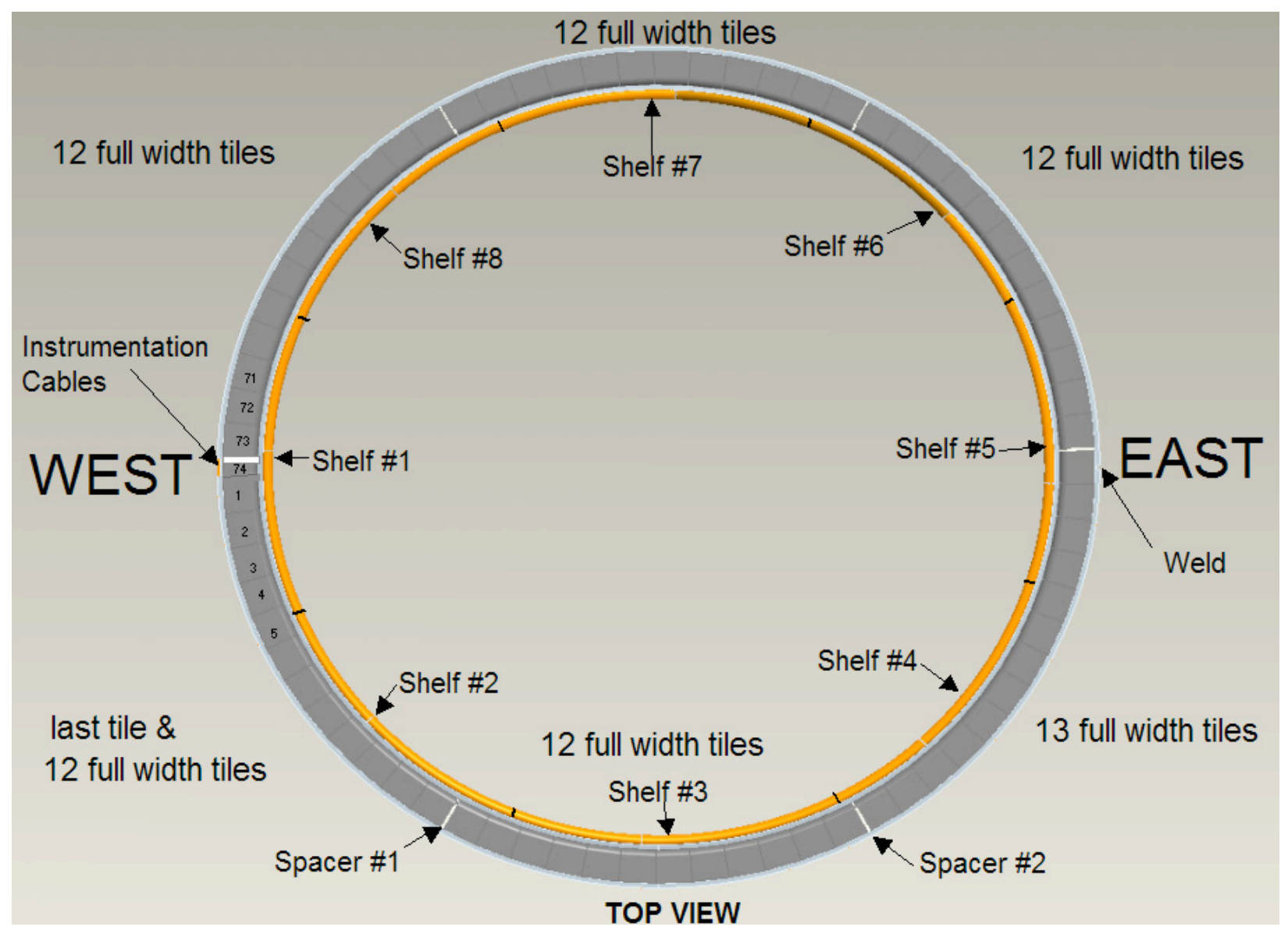

Figure 5.2 Layout of ferrite tiles, spacers, and shelves.

\subsubsection{Shelf System}

A set of 8 aluminum shelves support the ferrite tiles. Each shelf has a 7 degree angle on the cross section to match the ferrite tiles. The shelves are each screwed to the Marx skirt with a set of four $1 / 4-20$ socket head cap screws. Around each screw are 3 oval point 1032 setscrews for adjusting the shelf to match the profile of the Marx skirt and maintain the 7 degree angle. 
The inner radius of the shelves has a large radius rounded profile to prevent high voltage breakdown to the stalk. A finite element analysis was performed to confirm structural integrity of the shelf system. For a total weight of $1320 \mathrm{lbs}$, the maximum stress is 1344 psi (providing a safety factor of over 20), and the maximum deflection is 0.0023 inch (at the innermost radius location). For the 4 screws holding the shelf to the Marx skirt, a safety factor of 24 is provided for the full load applied to a single bolt. A load test on a mockup (primarily used for the oil bubble test--see Section 5.2) confirmed a safety factor of at least 3.6 with no plastic deformation.

The shelf at the west end has a 0.25 inch deep groove to accommodate routing 16 RG223 cables and the shelf at the east end has a similar groove to prevent interference with the weld bead in the Marx skirt.

\subsubsection{Diagnostic Loops in Wedge Pieces}

Two identical copper loops with an effective area of $0.72 \mathrm{~cm}^{2}$ (as measured in a calibration test by Jeff Johnson) are embedded in one of the double spacer pieces. These loops provide a measure of the instantaneous B field in the ferrite and also the voltage across the ferrite tiles. The leads exit the outer side of the spacer set and are routed along the same path as the existing 14 RG-223 cables. 


\begin{tabular}{|c|c|c|c|c|}
\hline Gap \# & $\begin{array}{l}\text { Between Tiles or } \\
\text { Spacers (S) }\end{array}$ & $\begin{array}{l}\text { Back, Top } \\
\left(\times 10^{-3} \text { in }\right)\end{array}$ & $\begin{array}{l}\text { Front, Top } \\
\left(\times 10^{-3} \text { in) }\right.\end{array}$ & $\begin{array}{c}\text { Front, Bottom (X } \\
10^{-3} \text { in) }\end{array}$ \\
\hline 1 & 1.74 & 0 & 0 & 0 \\
\hline 2 & 2.1 & 3 & 2 & 0 \\
\hline 3 & 3.2 & 0 & 1 & 0 \\
\hline 4 & 4.3 & 4 & 2 & 0 \\
\hline 5 & 5.4 & 6 & 2 & 0 \\
\hline 6 & 6.5 & 9 & 2 & 0 \\
\hline 7 & 7.6 & 6 & 1 & 1 \\
\hline 8 & 8.7 & 2 & 1 & 1 \\
\hline 9 & 9.8 & 3 & 1 & 0 \\
\hline 10 & 10.9 & 0 & 0 & 0 \\
\hline 11 & 11.10 & 12 & 2 & 0 \\
\hline 12 & 12.11 & 1 & 2 & 0 \\
\hline 13 & S1.12 & 0 & 0 & 0 \\
\hline 14 & 13.51 & 1 & 0 & 0 \\
\hline 15 & 14.13 & 0 & 0 & 0 \\
\hline 16 & 15.14 & 1 & 1 & 0 \\
\hline 17 & 16.15 & 0 & 1 & 0 \\
\hline 18 & 17.16 & 0 & 1 & 1 \\
\hline 19 & 18.17 & 0 & 1 & 1 \\
\hline 20 & 19.18 & 0 & 0 & 0 \\
\hline 21 & 20.19 & 1 & 2 & 0 \\
\hline 22 & 21.20 & 0 & 1 & 2 \\
\hline 23 & 22.21 & 14 & 0 & 1 \\
\hline 24 & 23.22 & 0 & 1 & 1 \\
\hline 25 & 24.23 & 0 & 0 & 1 \\
\hline 26 & S2.24 & 14 & 0 & 0 \\
\hline 27 & 25.52 & 7 & 0 & 0 \\
\hline 28 & 26.25 & 0 & 0 & 1 \\
\hline 29 & 27.26 & 0 & 0 & 1 \\
\hline 30 & 28.27 & 0 & 1 & 1 \\
\hline 31 & 29.28 & 10 & 0 & 1 \\
\hline 32 & 30.29 & 2 & 0 & 1 \\
\hline 33 & 31.30 & 0 & 0 & 3 \\
\hline 34 & 32.31 & 0 & 0 & 1 \\
\hline 35 & 33.32 & 1 & 0 & 2 \\
\hline 36 & 34.33 & 8 & 0 & 0 \\
\hline 37 & 35.34 & 0 & 0 & 1 \\
\hline 38 & 36.35 & 0 & 0 & 1 \\
\hline 39 & 37.36 & 0 & 0 & 1 \\
\hline 40 & S3.37 & 8 & 0 & 0 \\
\hline 41 & 38.53 & 4 & 0 & 0 \\
\hline 42 & 39.38 & 0 & 1 & 2 \\
\hline 43 & 40.39 & 8 & 1 & 0 \\
\hline 44 & 41.40 & 6 & 0 & 0 \\
\hline 45 & 42.41 & 5 & 0 & 1 \\
\hline 46 & 43,42 & 0 & 0 & 3 \\
\hline 47 & 44.43 & 12 & 0 & 1 \\
\hline 48 & 45,44 & 1 & 1 & 0 \\
\hline 49 & 46.45 & 10 & 1 & 1 \\
\hline 50 & 47.46 & 6 & 0 & 0 \\
\hline 51 & 48,47 & 4 & 1 & 0 \\
\hline 52 & 49.48 & 1 & 1 & 0 \\
\hline 53 & S4.49 & 10 & 0 & 0 \\
\hline 54 & 50.54 & 2 & 0 & 0 \\
\hline 55 & 51.50 & 7 & 1 & 0 \\
\hline 56 & 52.51 & 8 & 1 & 0 \\
\hline 57 & 53.52 & 0 & 1 & 0 \\
\hline 58 & 54.53 & 3 & 1 & 1 \\
\hline 59 & 55.54 & 0 & 1 & 1 \\
\hline 60 & 56.55 & 0 & 1 & 1 \\
\hline 61 & 57.56 & 0 & 1 & 1 \\
\hline 62 & 58.57 & 0 & 1 & 0 \\
\hline 63 & 59.58 & 0 & 0 & 0 \\
\hline 64 & 60.59 & 8 & 1 & 0 \\
\hline 65 & 61.60 & 0 & 1 & 1 \\
\hline 66 & S5.61 & 10 & 0 & 0 \\
\hline 67 & 62.55 & 10 & 0 & 0 \\
\hline 68 & 63.62 & 0 & 0 & 2 \\
\hline 69 & 64.63 & 1 & 0 & 1 \\
\hline 70 & 65.64 & 0 & 0 & 0 \\
\hline 71 & 66.65 & 0 & 1 & 1 \\
\hline 72 & 67.66 & 0 & 1 & 1 \\
\hline 73 & 68.67 & 9 & 1 & 0 \\
\hline 74 & 69.68 & 5 & 1 & 0 \\
\hline 75 & 70.69 & 3 & 1 & 1 \\
\hline 76 & 71.70 & 0 & 1 & 1 \\
\hline 77 & 72.71 & 0 & 1 & 1 \\
\hline 78 & 73.72 & 0 & 0 & 1 \\
\hline 79 & 56.73 & 0 & 0 & 0 \\
\hline 80 & 74,56 & 0 & 0 & 0 \\
\hline
\end{tabular}

Table 1. List of final as-measured gaps between tiles and spacers. Table provided by Russell Mitchell (LANL). 


\subsection{Oil Bubble Test}

One of the issues raised at the Interim Design Review was the issue of possible oil bubbles in the system and oil bubble migration to undesirable locations [8]. A two-tile mockup of the ferrite damper system was fabricated to study this particular issue. Figure 5.3 shows the oil bubble test hardware.

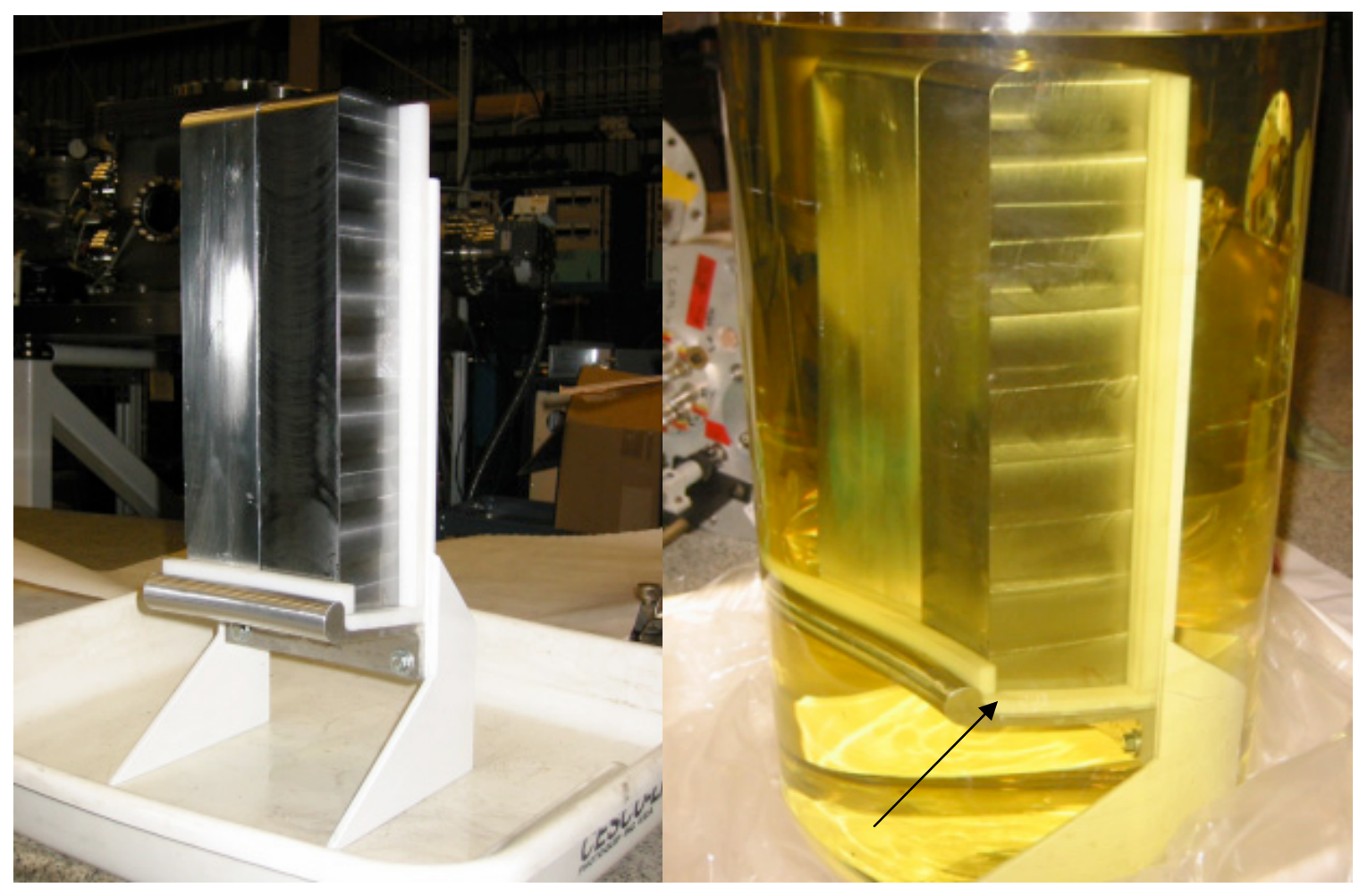

Figure 5.3 Oil test mockup. Right image shows components in oil.

The mockup assembly with 2 metal tiles (one steel and one aluminum) was placed inside a glass beaker and the beaker was filled with Diala AX oil by pumping the oil from a barrel directly into the beaker using a hose and a hand pump. The assembly was visually inspected for bubbles. Although there were many bubbles immediately after oil filling, no bubbles were observed along the bottom side of the shelf after 2.5 days. Several small bubbles were observed at the inner top area of the shelf (see arrow in Figure 5.3) even after 2.5 days, but had disappeared after 10 days. The design in this area was modified before installation to completely eliminate trapped bubbles in this area. 


\subsection{Installation}

Access to the area where the ferrite damper is installed was very limited. Installation procedures therefore needed to be fully developed and exercised to minimize the need for field modifications. The general installation procedure follows the steps outlined below:

a. Drill and tap one set of shelf installation holes in Marx skirt

b. Install aluminum shelf

c. Continue drilling and tapping shelf installation holes in Marx skirt

d. Continue installing aluminum shelves, route cables on west side through grooves in parts

e. Install bottom polyethylene and back polyethylene spacers

f. Install ferrite tiles and polyethylene spacers

g. Measure gap size for last ferrite tile

h. Cut last ferrite tile to size

i. Install last ferrite tile

j. Install double spacer wedge pieces

k. Connect diagnostic loop leads to cabling

\subsubsection{Work Platform}

A work platform was erected to provide stable, secure, and safe installation conditions for the technicians performing the installation. The work platform is a commercial Gentex scaffold with cutouts to accommodate the stalk and torlon rods supporting the Marx dome. Two Series 86 scaffolds are connected with a $6 \mathrm{X}$ extension platform. The overall dimensions of the work platform are 12 feet by 6 feet by 8 feet high. Scaffold training was required to perform work on the platform.

\subsubsection{Mockup Marx Skirt}

A full-scale mockup of the Marx skirt was fabricated out of mild steel. Practice installation procedures were conducted to identify problem areas and discuss methods to address these areas. A set of 18 ferrite tiles were installed onto the Marx skirt mockup. The practice installation procedures included:

- Drilling and tapping all the shelf installation holes

- Installing all aluminum shelves

- Installing bottom and back polyethylene spacers

- Installing 18 ferrite tiles

- Installing the double space wedge pieces (without diagnostic loops)

\subsubsection{Tooling}

Ferrite damper system installation involved several pieces of tooling:

- Shelf clamp (see Figure 5.4): these clamps provide 3 functions: 1) hold the conical bottom polyethylene spacers to the shelves, 2) radially press the 
polyethylene back spacers against the Marx skirt, and 3) provide an azimuthal stop for the ferrite tiles to press against during installation of the ferrite.

- Center punch jig (see Figure 5.5): this simple jig is used to locate the holes for drilling and tapping the Marx skirt prior to installation of the aluminum shelves. The drilling and tapping was performed with extreme care not to contaminate the area with metal shavings.

- Loading plate (see Figure 5.6): this is a pair of plates with a set of jack screws for applying azimuthal compression on the tile set. This device is used both to compress subsets of tiles against a shelf clamp as well as in the last tile slot (as shown in Figure 5.6). The tiles are wetted with oil during installation to reduce the friction between components to that of a fully oil-submerged system.

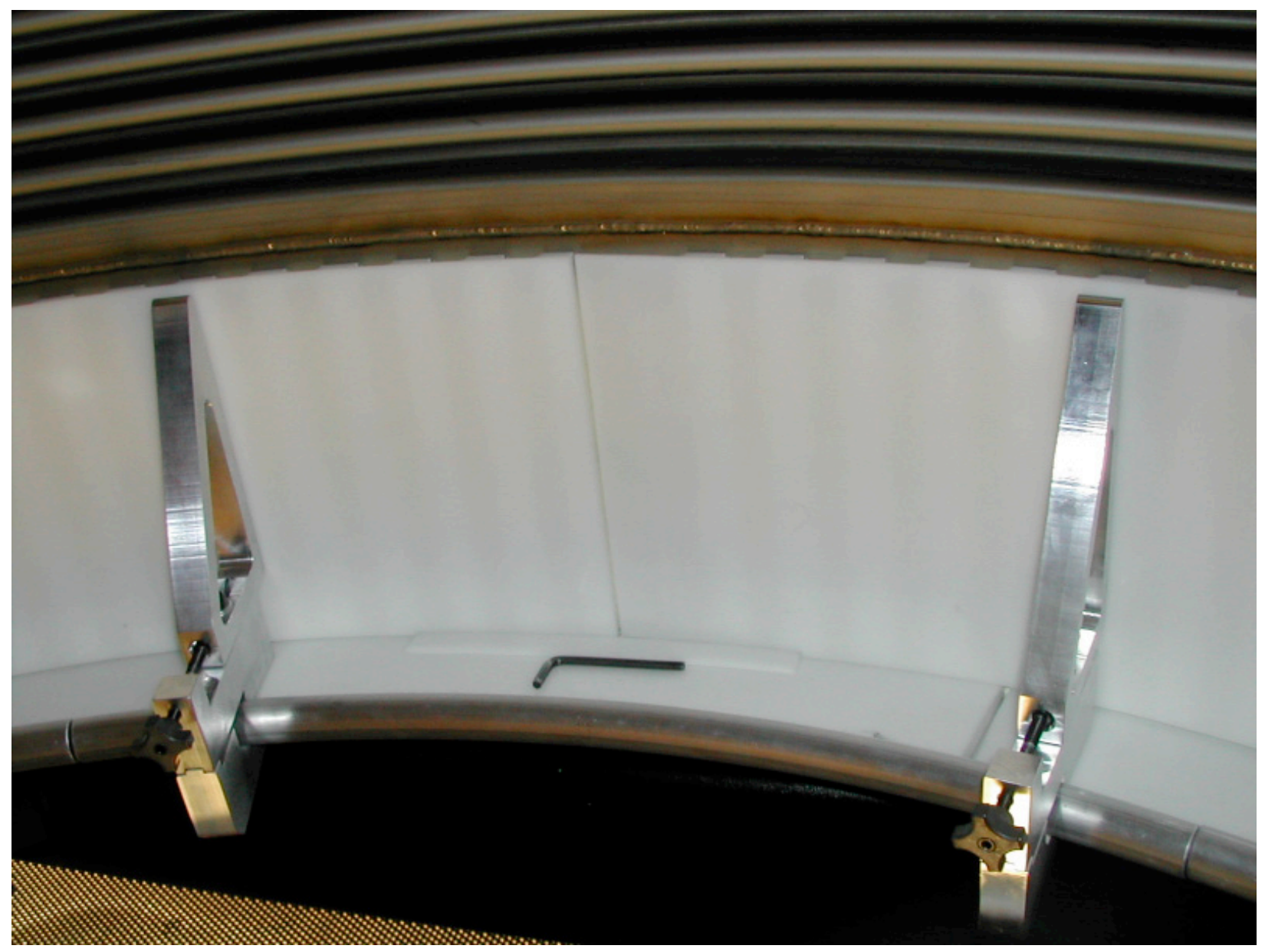

Figure 5.4 Two shelf clamps prior to ferrite tile installation. 


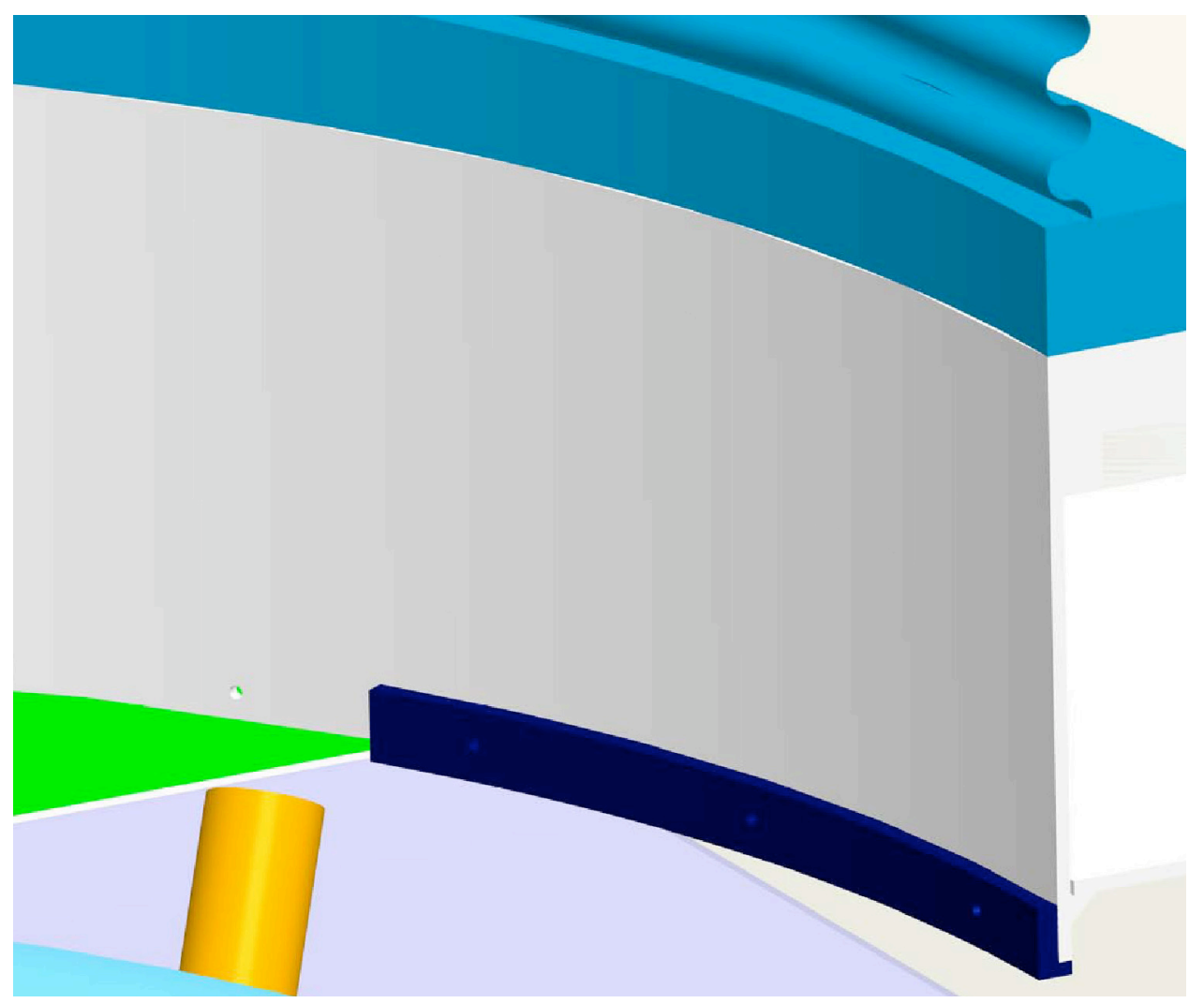

Figure 5.5 Center punch jig against Marx skirt (shown in blue). 


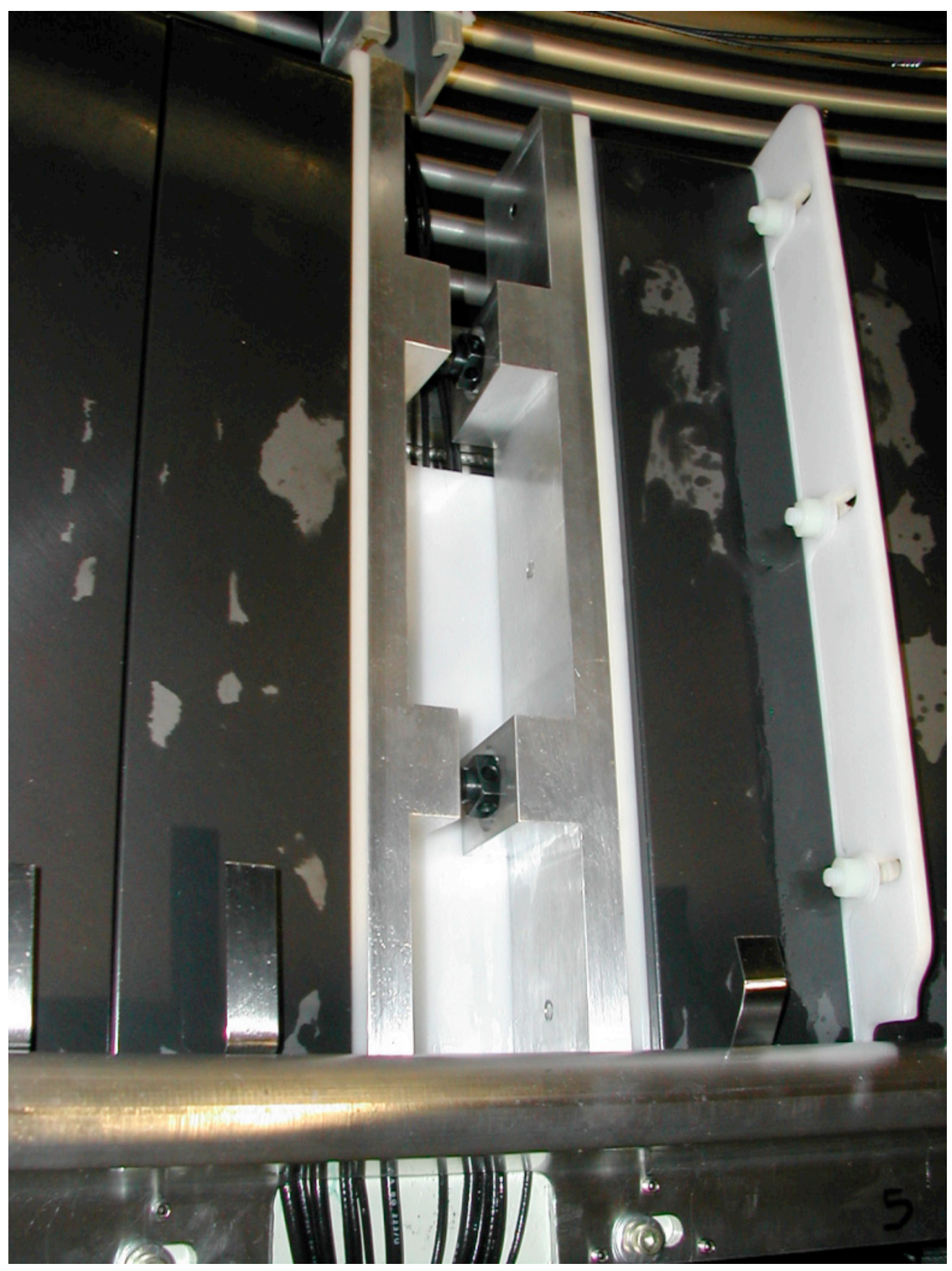

Figure 5.6 Loading plate at last space on west side. The double wedge spacer can be seen on the right side and the 16 RG223 cables can be seen behind the aluminum shelf at the bottom of the photo. The shims between each ferrite tile and the shelves are removed after assembly. 


\section{Ferrite Damper Performance}

In this Section, we discuss the observed damping of rise time induced $7.8 \mathrm{MHz}$ oscillations by the ferrite insert, which turns out to be in close accord with the predictions of the simulation circuit in (Section 4.3). To illustrate this and start with a concise "bottom line", a brief presentation of 7.8 MHz damping data before and after the ferrite insert installation is given in Section 6.1. More detailed analysis of data from a typical "no-beam" shot is covered in Section 6.2, and a similar analysis of data from a $\sim 1 \mathrm{kA}$ beam shot is presented in Section 6.3.

\subsection{Observed Damping by the Ferrite Insert}

The rise time induced $7.8 \mathrm{MHz}$ oscillations impact the beam dynamics in a couple of ways:

1. The beam voltage is modulated at $7.8 \mathrm{MHz}$, decaying from levels initially around $7-8 \%$ peak to peak, as observed on the source dome E-Dot monitor (see Section 3.3).

2. The beam transverse position oscillates at $7.8 \mathrm{MHz}$. The amplitude of these $\mathrm{mm}$ scale transverse beam deflections is consistent with the amplitude of the transverse RF magnetic fields in the diode region estimated from the stalk current (Rogowski) monitors and the vacuum B-Dot loops in the injector.

Shots with a cold cathode ("no beam" shots) provide the most direct and quantitative measure of the ferrite insert's contribution to the damping of the $7.8 \mathrm{MHz}$ oscillations. In Figure 6.1, the source dome E-Dot signal from a typical "no beam" shot before the installation of the ferrite is compared with a source dome E-Dot signal after the ferrite was installed. Note that the voltage fluctuations with the ferrite damper installed decay to an insignificant level $(<1 \%)$ in about $\sim 0.5 \mu \mathrm{sec}$. (The flat top voltage level is slightly lower in the shot with the damper installed, but this does not have a significant effect on the damping rate.)

A similar comparison of the signals from the B-Dot \#3 monitors over a 2 to $4.5 \mu \mathrm{sec}$ window for the same two shots is shown in Figure 6.2. To obtain a quantitative measure of the damping rate, the magenta line in the figure provides a fit of the amplitude decay to an exponential curve. The previously observed decay time constant of $\sim 0.8 \mu \mathrm{sec}$ has been reduced to $\sim 0.25 \mu \mathrm{sec}$ by the addition of the ferrite damper, an increase in the decay rate by more than a factor of 3 .

With a hot cathode and beam extraction from the diode, the beam loading also contributes to the damping of the oscillations as discussed in detail in Section 3.3. The 
data from a typical beam shot with the ferrite damper installed is presented in Section 6.3; the bottom line is a reduction of the decay time from $\sim 0.5 \mu \mathrm{sec}$ (with $\mathrm{a} \sim 1 \mathrm{kA}$ beam) to $\sim 0.2 \mu \mathrm{sec}$ by the ferrite damper.

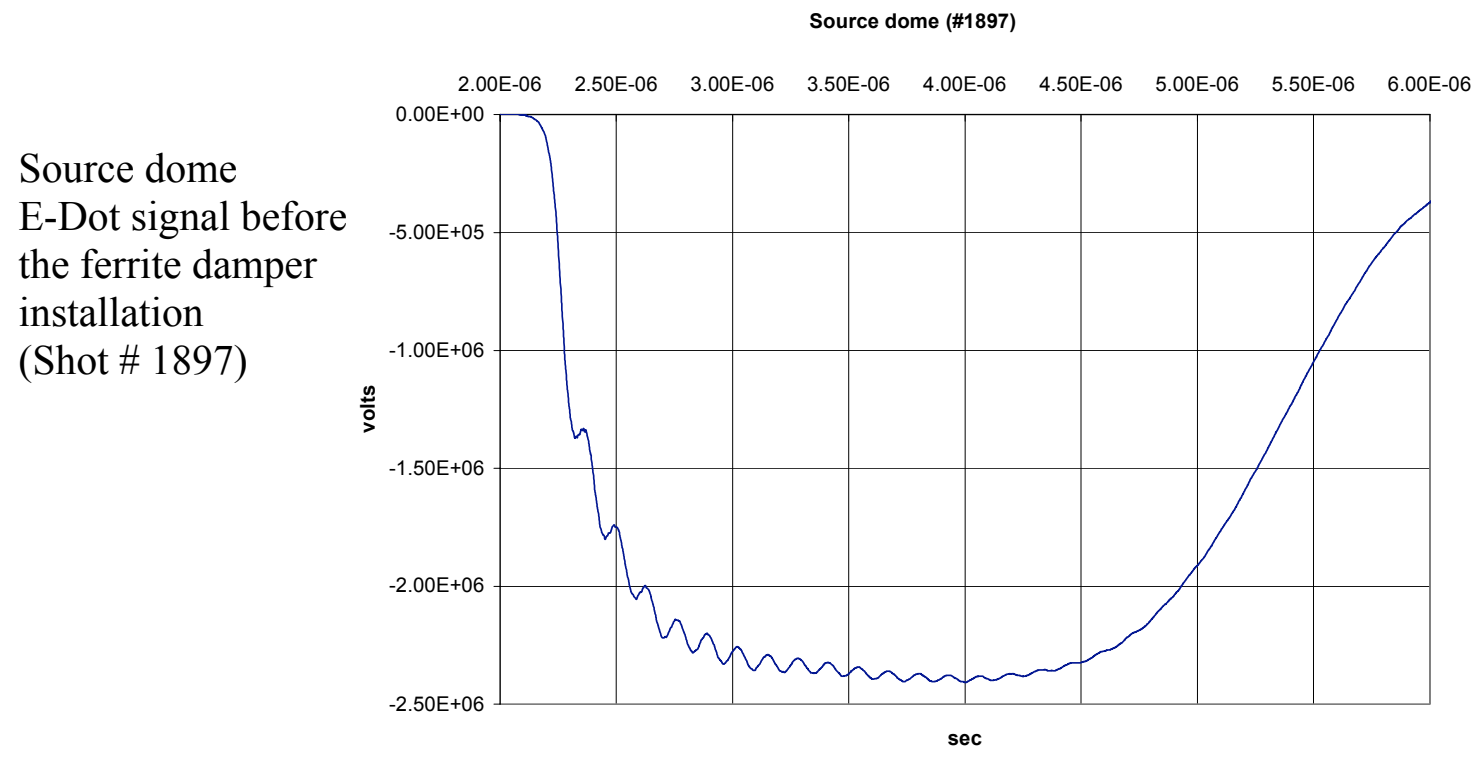

Source dome

E-Dot signal after the ferrite damper installation (Shot \# 3064)

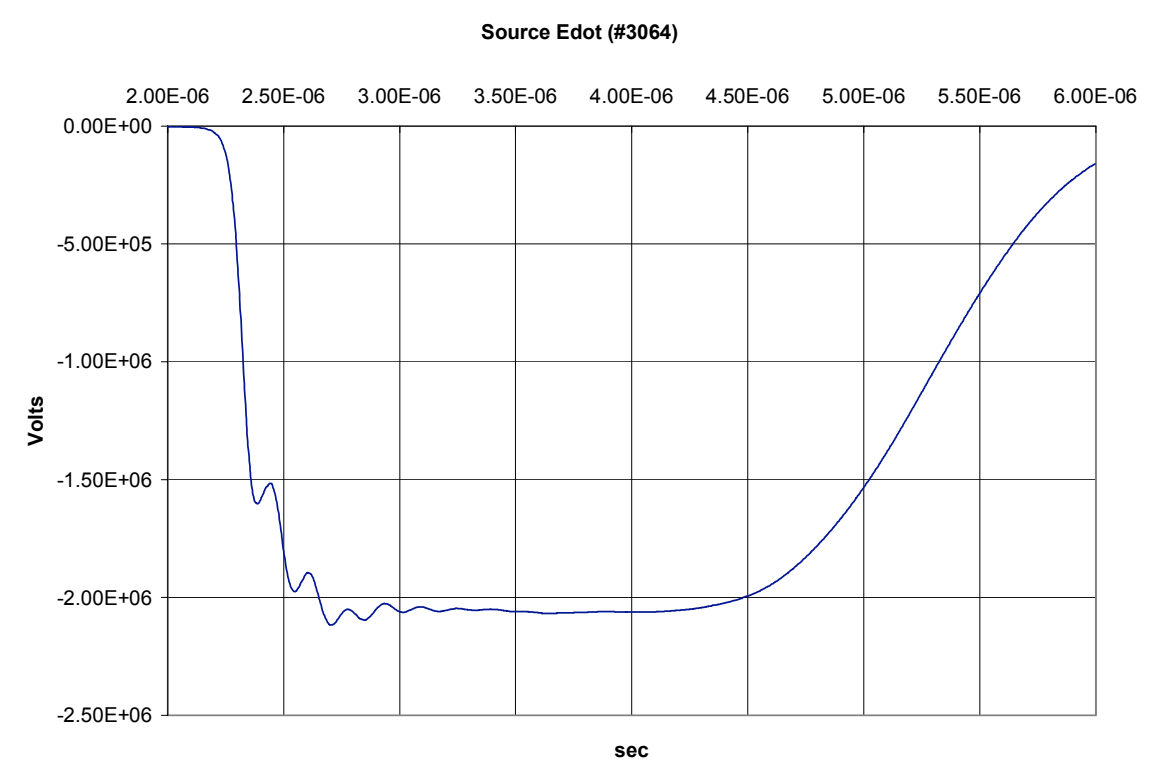

Figure 6.1 Comparison of source dome voltage monitors before and after the installation of the ferrite damper for two typical "no beam" shots. 
B-Dot\#3 signal before ferrite installation (Shot \# 1897)

oscillation frequency $=$ $7.8 \mathrm{MHz}$

oscillations decay with an e-folding time constant $\sim 0.8$ usec

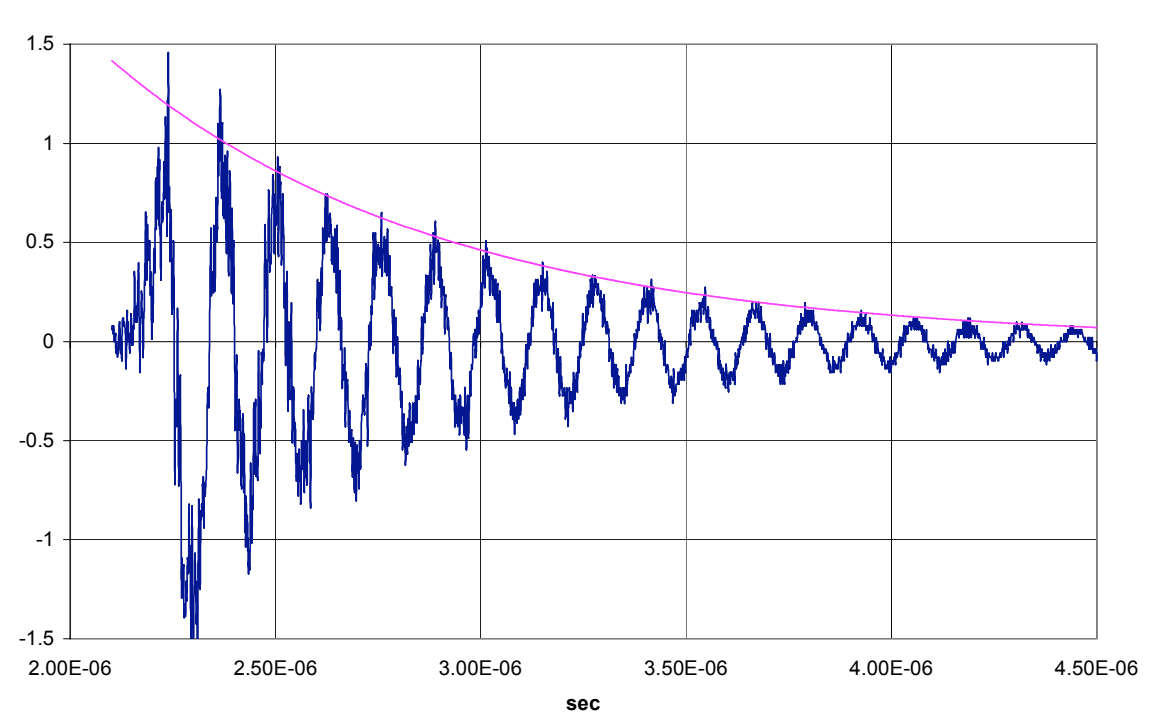

B-Dot\#3 signal after ferrite installation (Shot \# 3064)

oscillation frequency $=$ $6.1 \mathrm{MHz}$ oscillations decay with an e-folding time constant $\sim 0.25$ usec

Bdot \#3 (\#1897)

Bdot \#3 (\#3064)

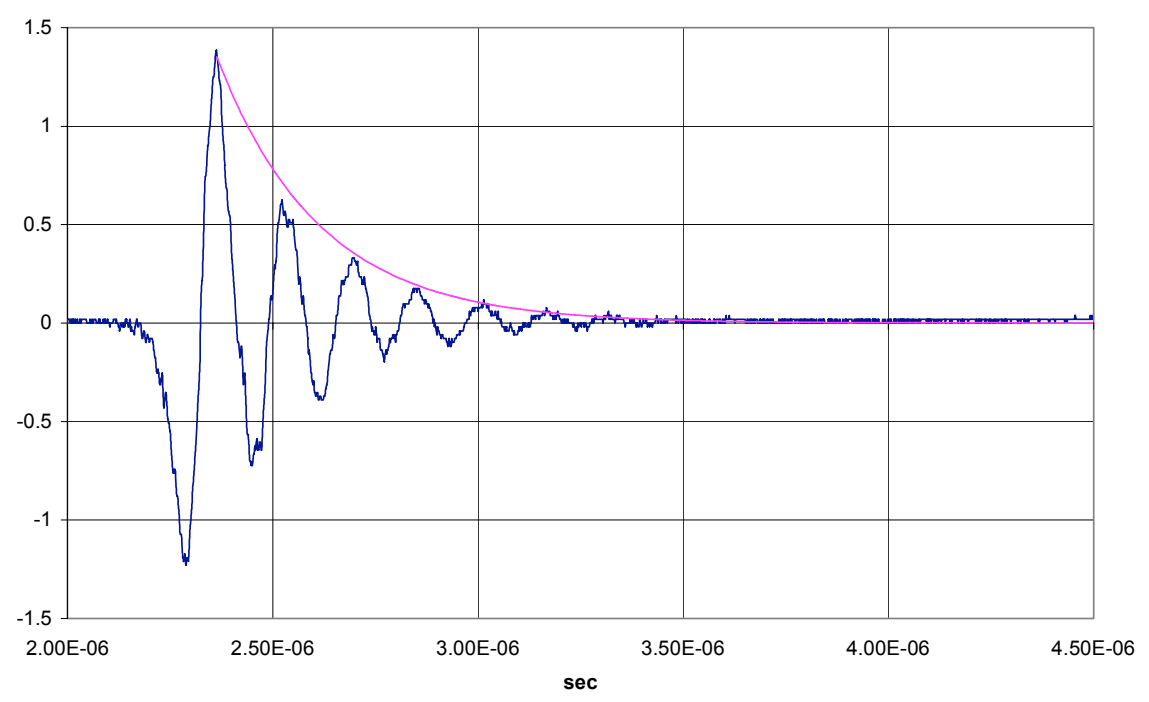

Figure 6.2 Comparison of RF loop monitors in the injector (B-Dot\#3) before and after the installation of the ferrite damper for the same two typical "no beam" shots as Figure 6.1. The magenta line is a fit of the amplitude decay to an exponential curve. 
A summary of the oscillation decay times with and without the ferrite damper for "no beam" and $\sim 1 \mathrm{kA}$ beam shots are summarized in Table 6.1. Note that the damping times with the ferrite damper installed are essentially the same as those measured in the scaled simulation circuit discussed in Section 4.3 (although the scaled ferrite area and scaled gap width were both about $20 \%$ less in the simulation circuit than in the actual damper).

\begin{tabular}{|lll|}
\hline & No ferrite damper & With ferrite damper \\
\hline No beam (cold cathode) & $0.8 \mu \mathrm{sec}$ & $0.25 \mu \mathrm{sec}$ \\
\hline With $\sim 1 \mathrm{kA}$ beam & $0.5 \mu \mathrm{sec}$ & $0.2 \mu \mathrm{sec}$ \\
\hline
\end{tabular}

Table 6.1 Summary of oscillation decay times comparing data before and after the installation of the ferrite damper.

\subsection{Analysis of a "No Beam" Shot (\#3064)}

The source dome voltage for shot \#3064 is shown in Figure 6.1. In Figure 6.3, the column CVR signal for this shot is shown. Note that the rise time oscillations are now apparent on this diagnostic, in contrast to the column CVR data before the ferrite damper was installed (see Fig 3.2). This observation is consistent with the discussion in Section 3.5: the $7.8 \mathrm{MHz}$ mode had a voltage null around the bottom of the column, so the $7.8 \mathrm{MHz}$ RF on the CVR was minimal (see Figure 3.20). With the ferrite installed, there is a significant shift in the oscillation frequency as discussed below, and the voltage null is no longer at the bottom of the column.

The column CVR asymptotes to a steady current of around $360 \mathrm{amps}$ in the latter part of the pulse flat top. This steady state current is roughly consistent with its expected value for a $\sim 2 \mathrm{MeV}$ source dome voltage and a column resistance of $5.3 \mathrm{ohms}$. 


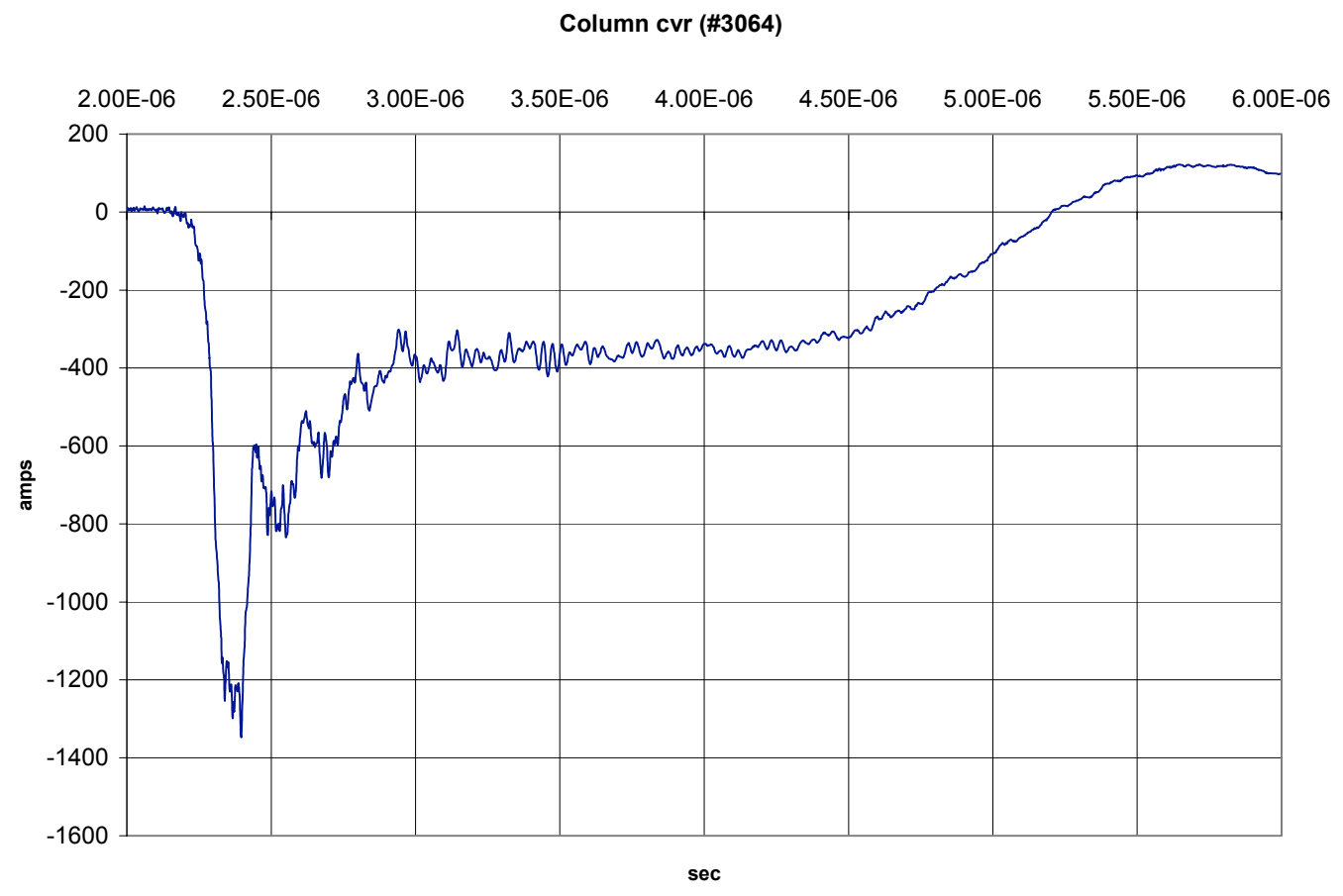

Figure 6.3 Column CVR signal for shot \#3064.

The stalk Rogowski signal is shown in Figure 6.4. (The processed signal amplitude was adjusted to compensate for a calibration and/or attenuator error that has not been identified at this time.) The magnetic field in the ferrite damper is shown in Figure 6.5 (arbitrarily defined as positive). Note that the $\mathrm{B}$ field in the ferrite is driven close to saturation $(\sim 0.3 \mathrm{~T})$ by the initial $\sim 6 \mathrm{kA}$ current spike flowing up the stalk. The amplitude of this magnetic field spike is consistent with what was expected on the basis of the scaled simulation circuit tests discussed in Section 4.3. 
Stalk Rogowski (\#3064)

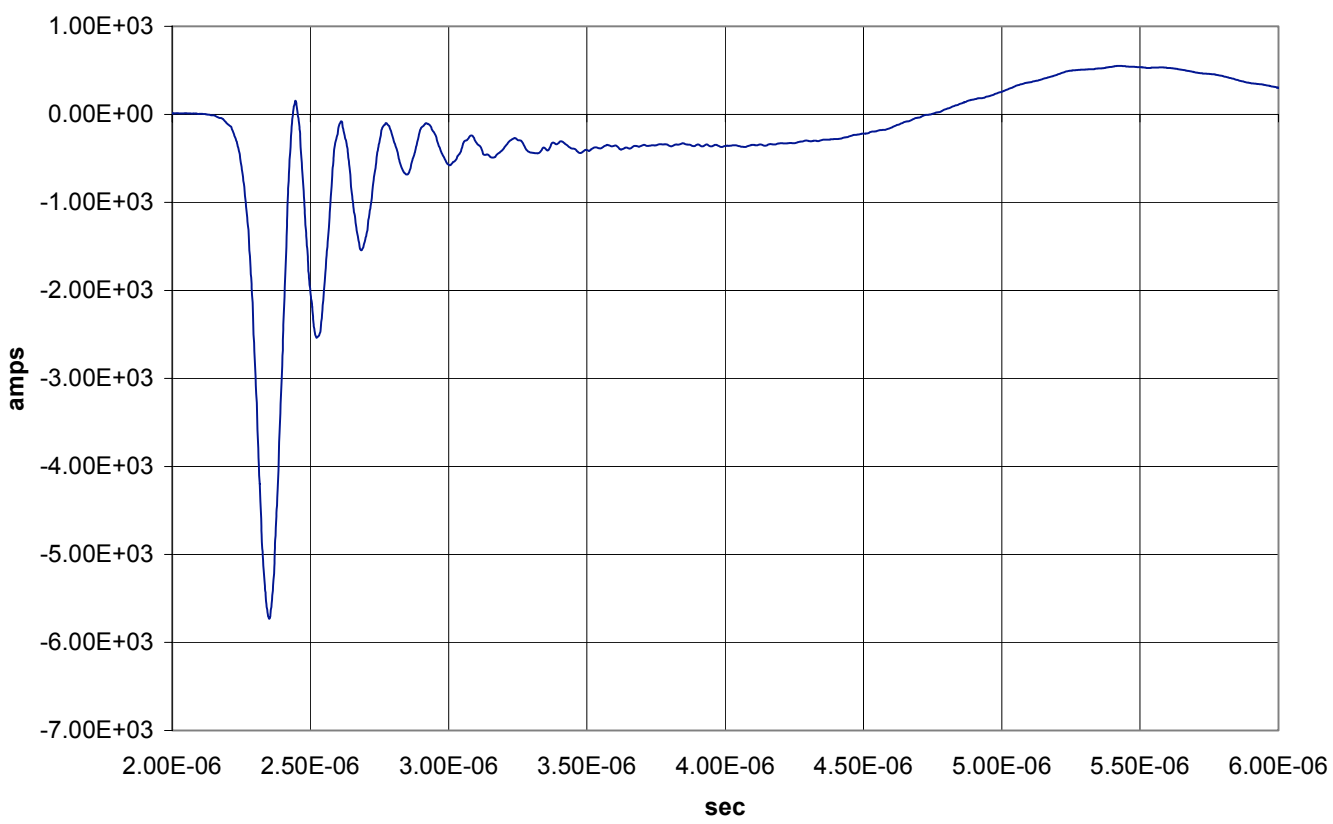

Figure 6.4 Stalk Rogowski signal for shot \#3064.

Note also that the magnetic field in the ferrite asymptotes to a steady value of $22 \mathrm{mT}$ after the oscillations die out. It is interesting to check whether this value is consistent with what would be expected. In the asymptotic steady-state regime, the effective $\mu$ of the ferrite is very large. From Eq. (4.3) in Section 4.2, if

$$
\mu / \mu_{0} \gg>2 \pi r / \Delta \sim 250
$$

where $r \sim 80 \mathrm{~cm}$ is the mean radius of the ferrite torus and $\Delta \sim 2 \mathrm{~cm}$ is the sum of all the gap widths, then the steady-state $B$ field in the ferrite is given by

$$
B=\frac{\mu_{0} I}{\Delta}
$$


Using the steady-state stalk current of 360 amps deduced from the column CVR data and a sum of all the gap widths of $2 \mathrm{~cm}$, we predict a steady-state B field of $22.6 \mathrm{mT}$, consistent with the ferrite loop measurement.

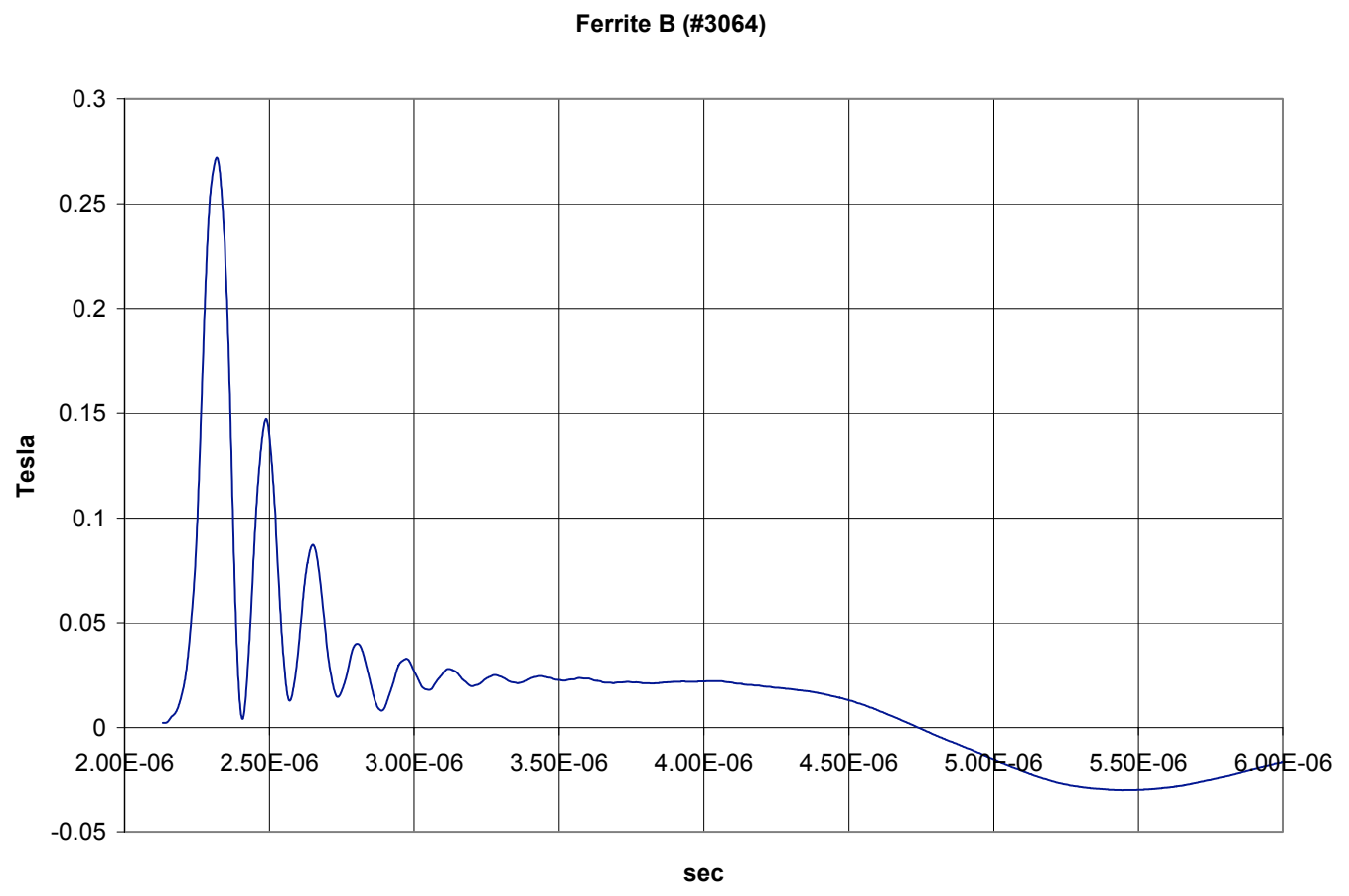

Figure 6.5 Ferrite B field signal for shot \#3064.

The ferrite loop data shown in Figure 6.5 is the "processed signal", an integral of the loop voltage output divided by the (calibrated) loop area of $0.72 \mathrm{~cm}^{2}$ to derive the magnetic field. The "raw" ferrite loop voltage data is also of interest since it provides a measure of the total voltage across the ferrite torus when multiplied by the ratio of the ferrite torus area $\left(210 \mathrm{~cm}^{2}\right)$ to the loop area. This ferrite voltage signal is presented in Figure 6.6 over a $2-3.4 \mu \mathrm{sec}$ time window (the front half of the pulse). The observed peak value of the ferrite damper voltage pulse around $-120 \mathrm{kV}$ is roughly consistent with expectations from the scaled circuit tests discussed in Section 4.3.

For easy comparison, the stalk Rogowski signal in this same time window is presented in Figure 6.7. (Note: The relative timing of the ferrite voltage and stalk current have not been determined at this time; the cable lengths from the ferrite voltage monitors were cut to fit and not to keep constant timing.) 
Ferrite voltage (\#3064)

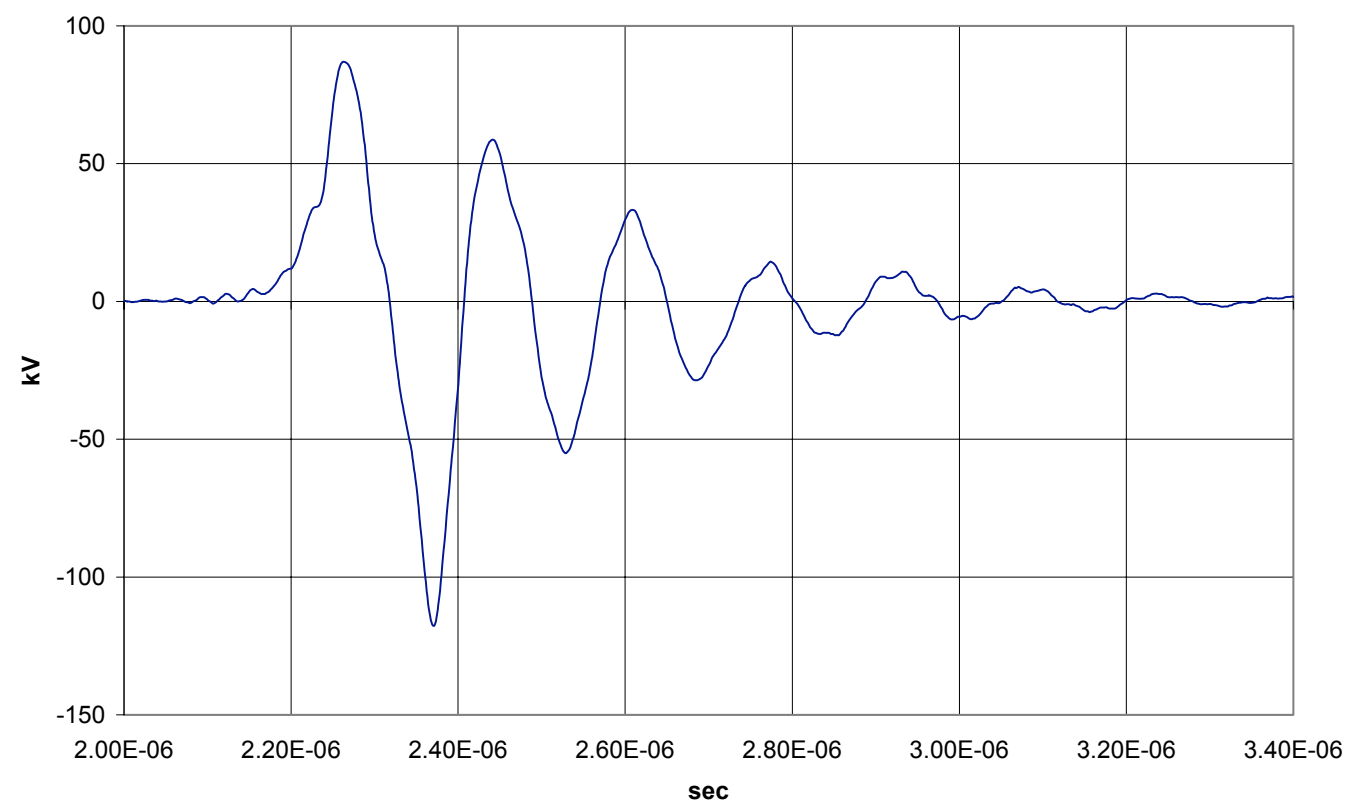

Figure 6.6 Voltage across the ferrite damper in the first half of the pulse.

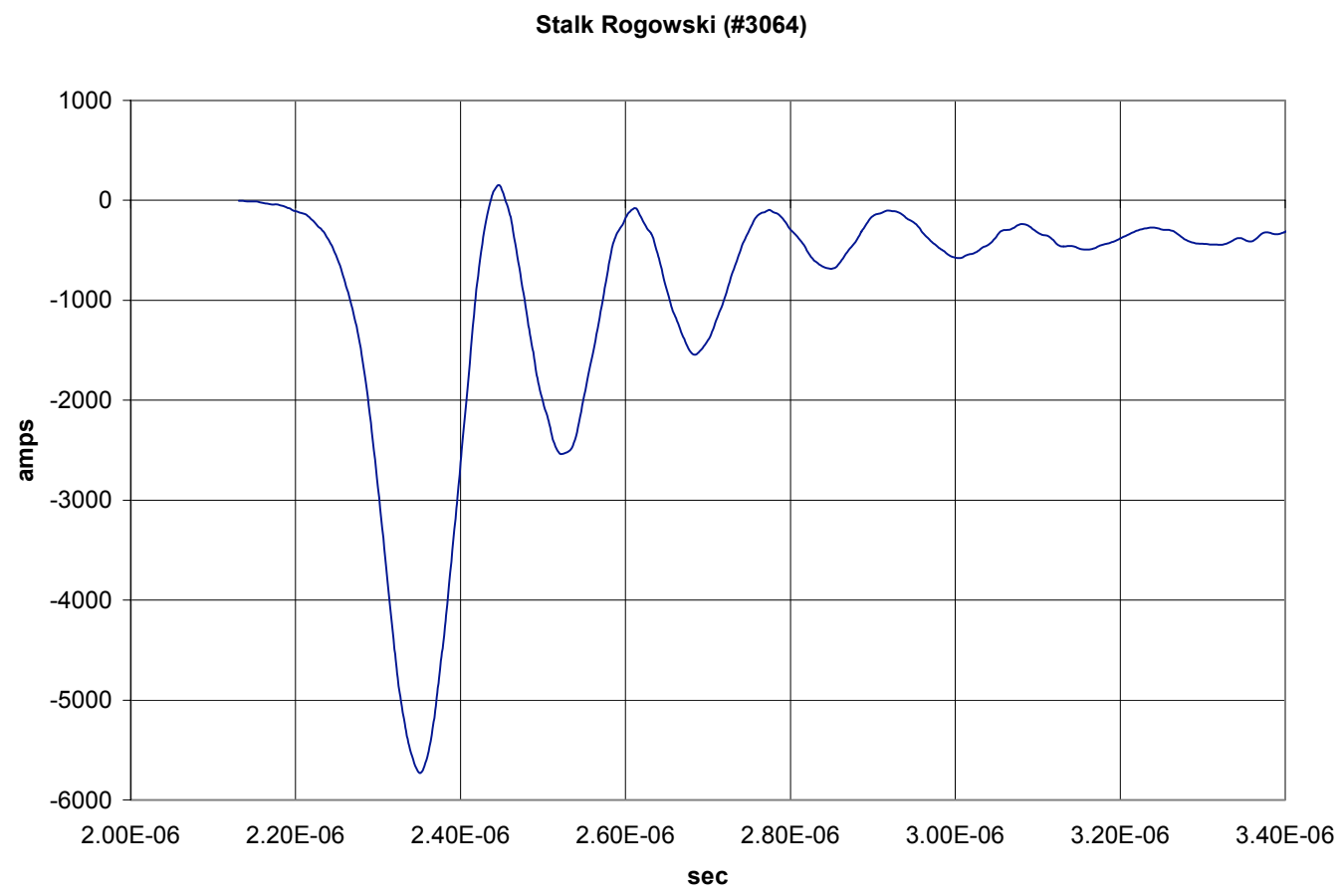

Figure 6.7 Stalk (Rogowski) current in the first half of the pulse.

The B-Dot \#3 loop signals in Figure 6.2 clearly show the enhanced damping with the ferrite insert, but they also indicate a significant frequency shift in the rise time induced 
oscillations. As illustrated more clearly in the FFT spectra of B-Dot \#3 in Figure 6.8, the oscillations are now centered around $6.1 \mathrm{MHz}$, a $30 \%$ shift in the resonant frequency. This shift is much larger than the $\sim 15 \%$ shift observed in the simulation circuit tests.

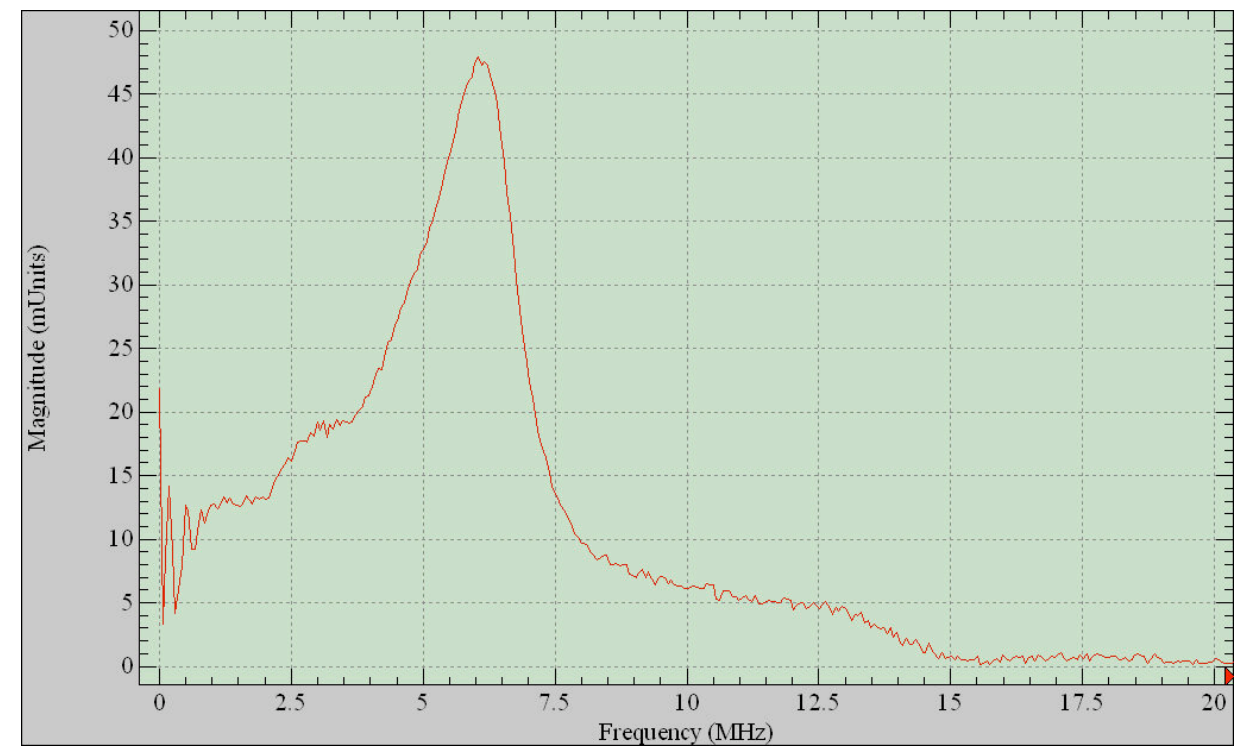

Figure 6.8 FFT spectra of B-Dot \#3 loop signal for shot \# 3064.

The ferrite impedance that determines the oscillation damping rate and the frequency shift is nonlinear and dispersive, as emphasized in the discussion of the damper design in Section 4. This nonlinearity is evident, for example, in the non-sinusoidal nature of the ferrite voltage waveform in Figure 6.6. A close look at the decay of the oscillations in Figure 6.2 also suggests a faster decay in the first cycle or two followed by a slower decay, so the "fit" to an exponential was only meant to provide a global measure of the damping rate.

It is useful, nonetheless, to see if a simple linear lumped circuit model of the injector like the one discussed at length in Section 3.3 can provide a rough explanation of the observations. In this model one might be tempted to include the ferrite damper as a lossy inductor and leave the source and Marx end capacitances unchanged. This simple model would require an added ferrite inductance of $1.7 \mu h$ to explain the observed frequency shift. This inductance is larger than the upper limit for the ferrite inductance obtained with a $2 \mathrm{~cm}$ gap and $\mu \rightarrow \infty$ of about $1.3 \mu \mathrm{h}$. A more plausible simple lumped model would recognize a shift in the voltage null away from the stalk midplane, which means a much larger fraction of the upper stalk capacitance should be added to the source dome capacitance. A simple estimate indicates that an increase in the effective net series $\mathrm{C}$ (in the C-L-C circuit model) from this change in the mode pattern might be of order $15 \%$, reducing the required series inductance increase from the ferrite insert to $\sim 1 \mu \mathrm{h}$ to explain the observed frequency shift. 
An effective ferrite inductance of order $1 \mu \mathrm{h}$ corresponds to a reactance of order 40 ohms at the resonant frequency. This is a reasonable impedance level from the observed ferrite voltage and stalk current waveforms $(200 \mathrm{kV}$ peak-to peak ferrite voltages driven by 5-6 kA peak-to peak stalk current swings). The observed damping rate, a time constant of about $0.25 \mu \mathrm{sec}$, is also consistent with an effective series resistance of the ferrite of about 30 ohms. These estimates are not meant to serve as "explanations" for the observations, of course, but simply as useful cross-checks on the general consistency of the voltages, currents, frequency shifts, etc.

\subsection{Analysis of a $1 \mathrm{kA}$ Beam Shot (\# 3054)}

The source dome voltage waveform for shot \# 3054 is presented in Figure 6.9. As expected, the diode voltage fluctuations are even more strongly damped with a beam, dropping below the $\sim 0.5 \%$ level in about $\sim 0.5 \mu \mathrm{sec}$.

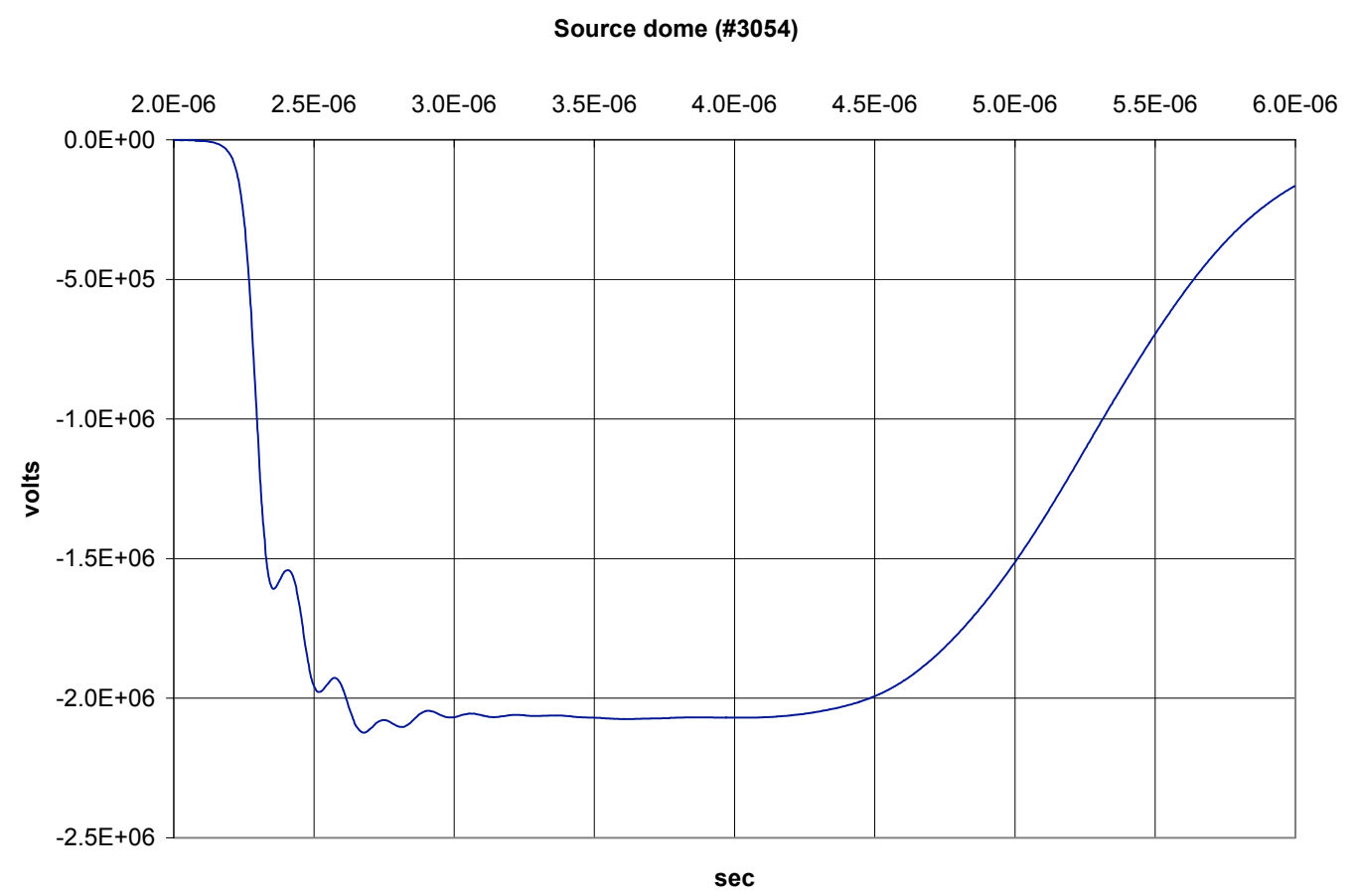

Figure 6.9 Source dome voltage for shot \# 3054 .

The Rogowski stalk current and the column CVR signal for this shot are presented in Figure 6.10 and Figure 6.11, respectively. (Once again, the processed signal amplitude on the Rogowski was adjusted to roughly compensate for a calibration and/or attenuator error that has not been identified at this time.) The column current is essentially the same as in the "no beam" shot, while the Rogowski asymptotes to a steady state value of $\sim 1.3$ $\mathrm{kA}$, the sum of the steady column current and the beam current. 


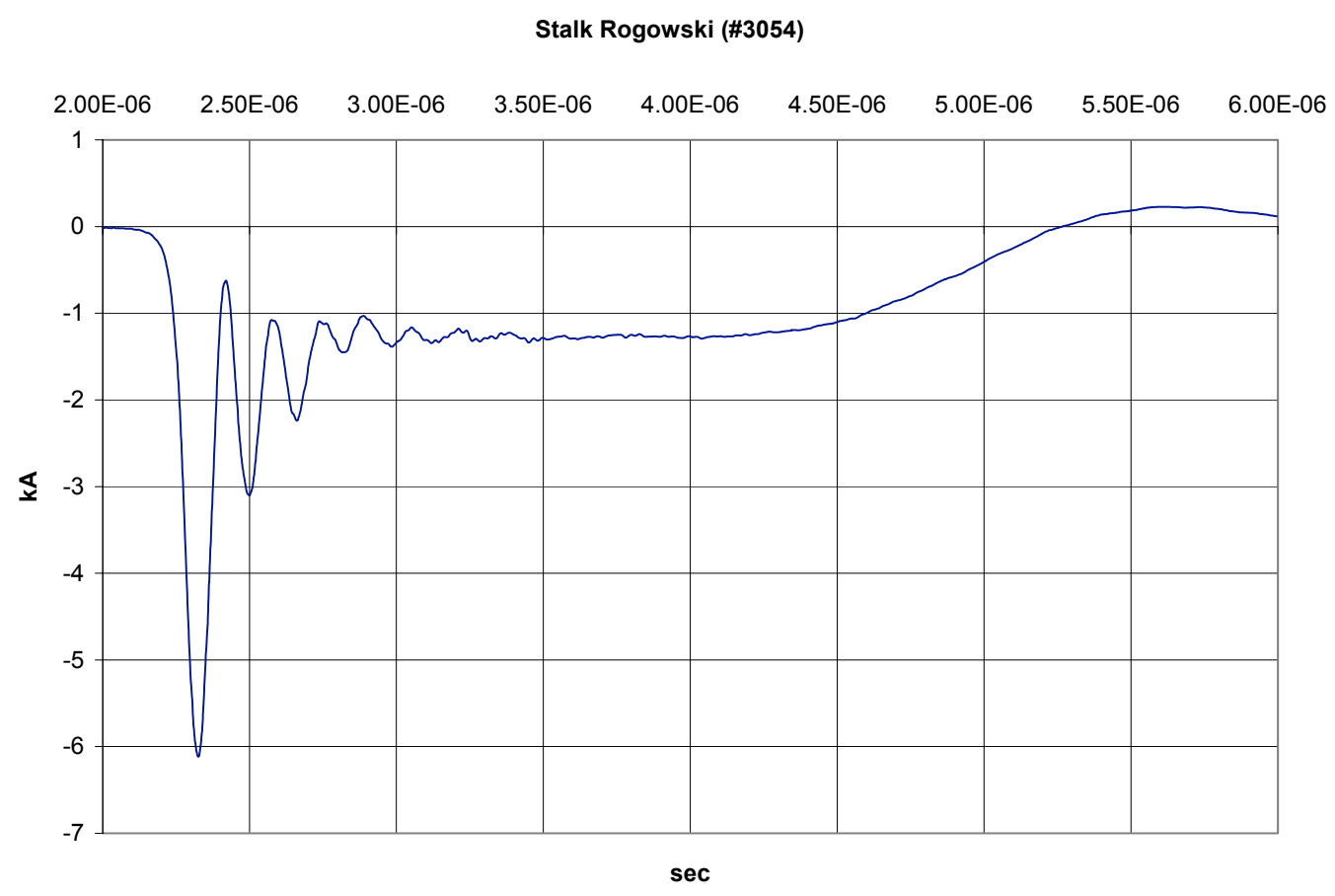

Figure 6.10 Rogowski stalk current for shot \# 3054.

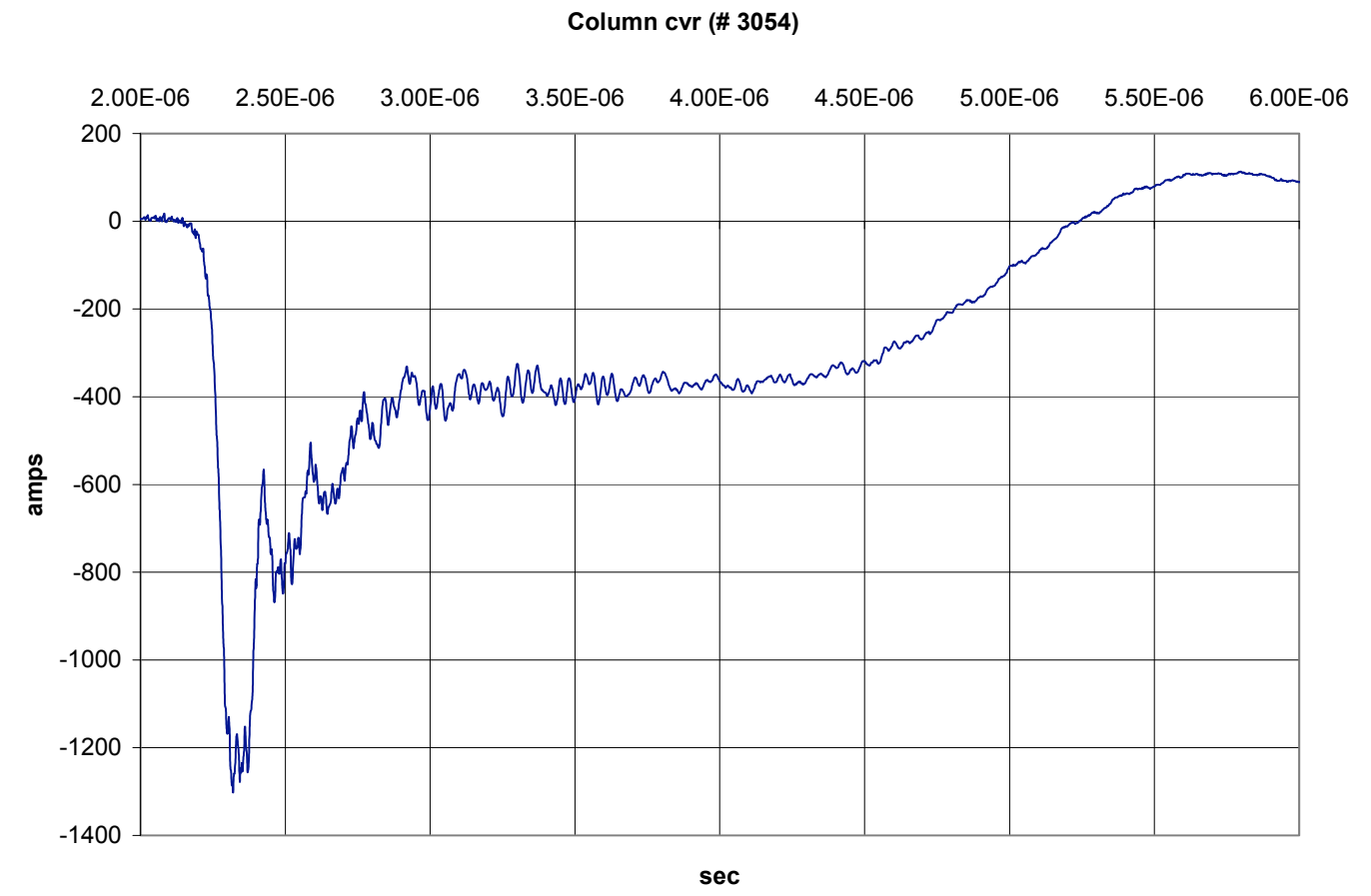

Figure 6.11 Column CVR signal for shot \# 3054 .

The magnetic field in the ferrite damper for this shot is shown in Figure 6.12. The B field in the ferrite is again driven close to saturation $(\sim 0.3 \mathrm{~T})$ by the initial $\sim 6 \mathrm{kA}$ current spike flowing up the stalk. The B field now asymptotes to a steady state value around 70 
$\mathrm{mT}$ in the flat top, driven by a steady state stalk current that is 3.6 times higher than in the "no beam" shot. This steady state B field increases a little less than linearly with the

Ferrite B (\#3054)

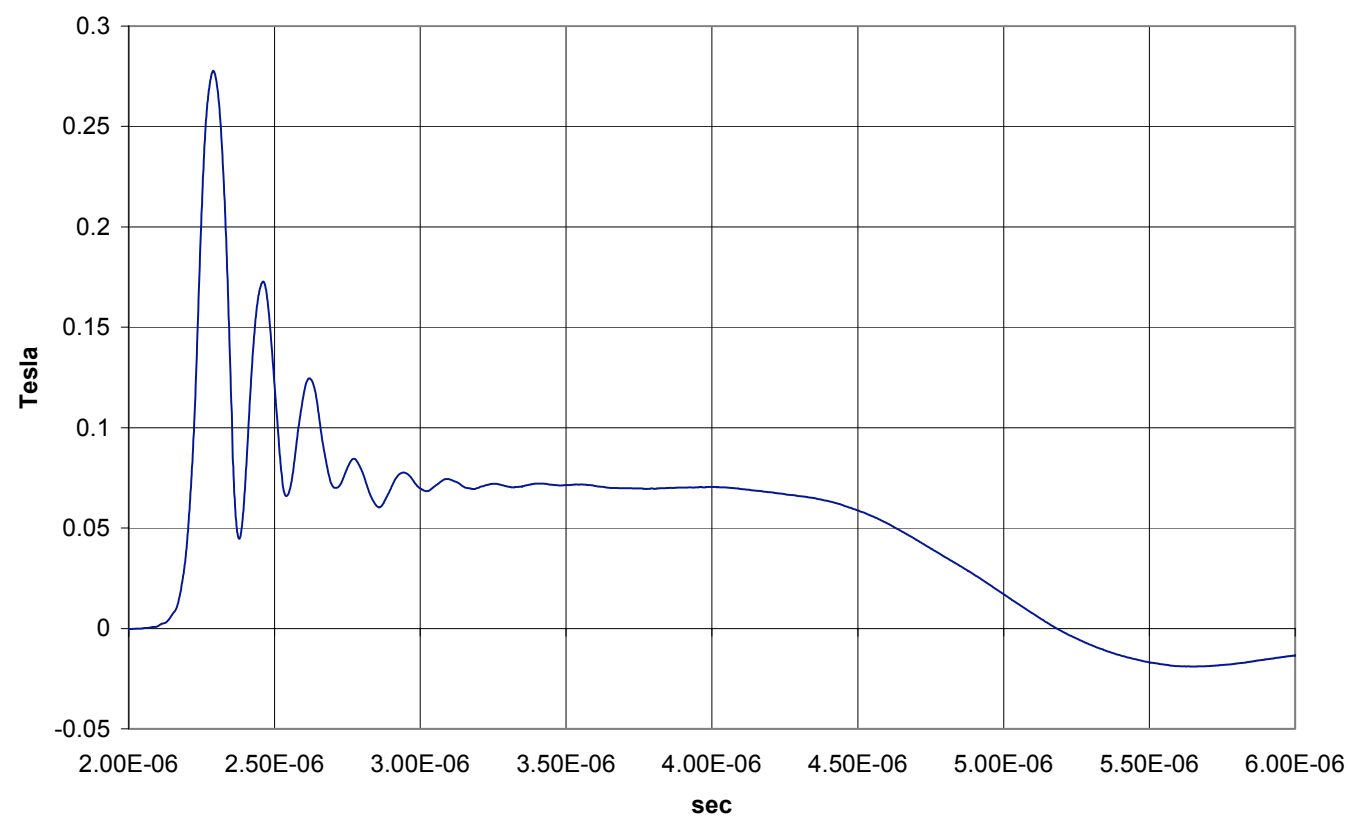

Figure 6.12 Ferrite B field signal for shot \# 3054 .

increased stalk current $(70 / 22 \sim 3.2)$; the likely explanation is a reduced $\mu$ at the higher bias point that makes the approximation in Eq. (6.1) less valid.

The voltage across the ferrite damper over a $2-3.4 \mu \mathrm{sec}$ time window (roughly the front half of the pulse) is shown in Figure 6.13. The non-sinusoidal nature of the ferrite voltage waveform from its nonlinear response is again very evident. The peak voltage in now about $100 \mathrm{kV}, 20 \%$ less that it was in the comparable "no beam" shot, although the peak-to-peak initial voltage swing is about the same.

The B-Dot \#3 RF loop signal is presented in Figure 6.14. The red line is a rough fit of the amplitude decay to an exponential curve, with an e-folding time constant $\sim 0.2 \mu \mathrm{sec}$. Prior to the installation of the ferrite damper, the decay of the rise time induced $7.8 \mathrm{MHz}$ oscillations with a $1.3 \mathrm{kA}$ beam was $\sim 0.5 \mu \mathrm{sec}$ (e.g., see Figure 3.10 ).

The reduction of the time constant without beam $(0.25 \mu \mathrm{sec})$ to $\sim 0.2 \mu \mathrm{sec}$ with $\mathrm{a} \sim 1$ kA beam current is roughly what one would expect even without considering the ferrite 
Ferrite voltage (\# 3054)

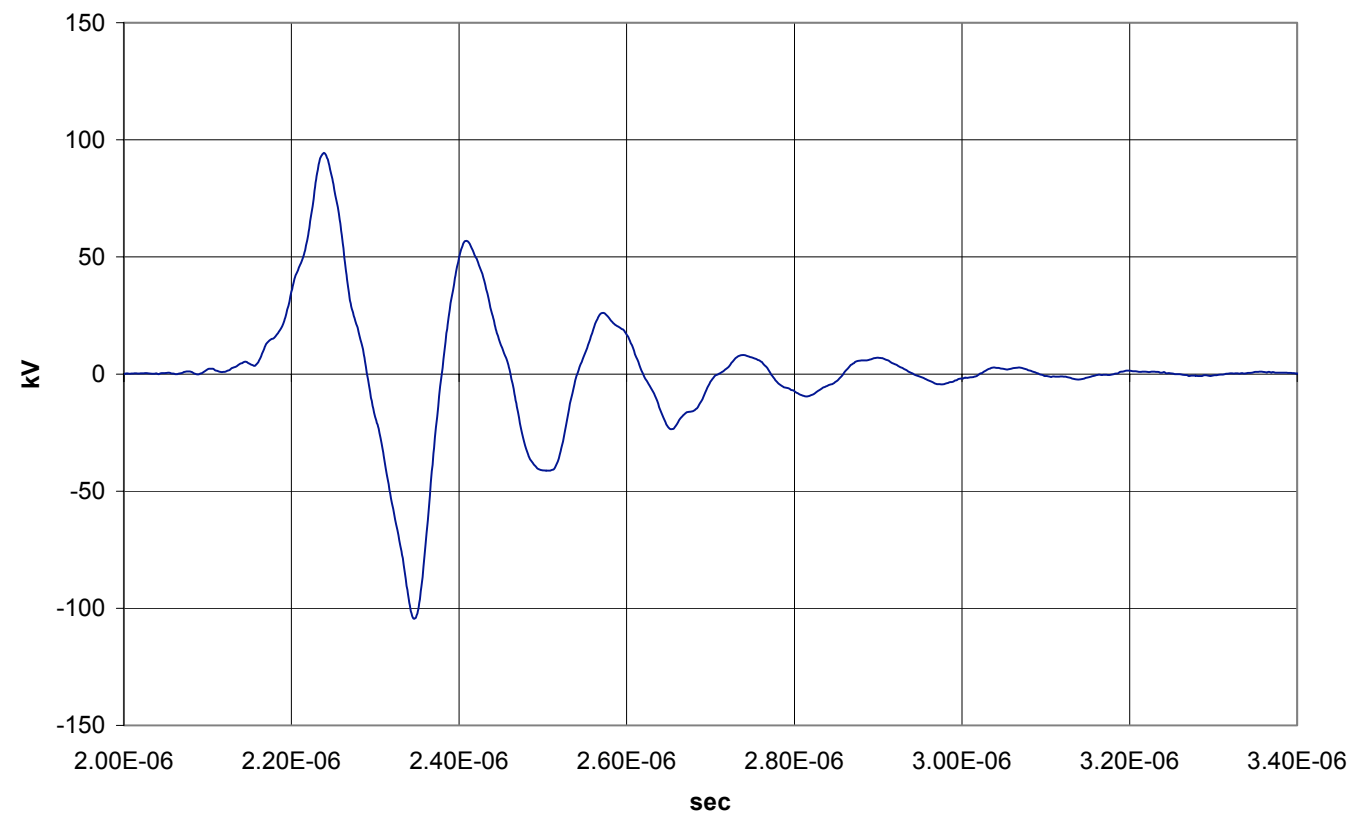

Figure 6.13 Voltage across the ferrite damper in the first half of the pulse for shot \# 3054.

Bdot\#3 (\#3054)

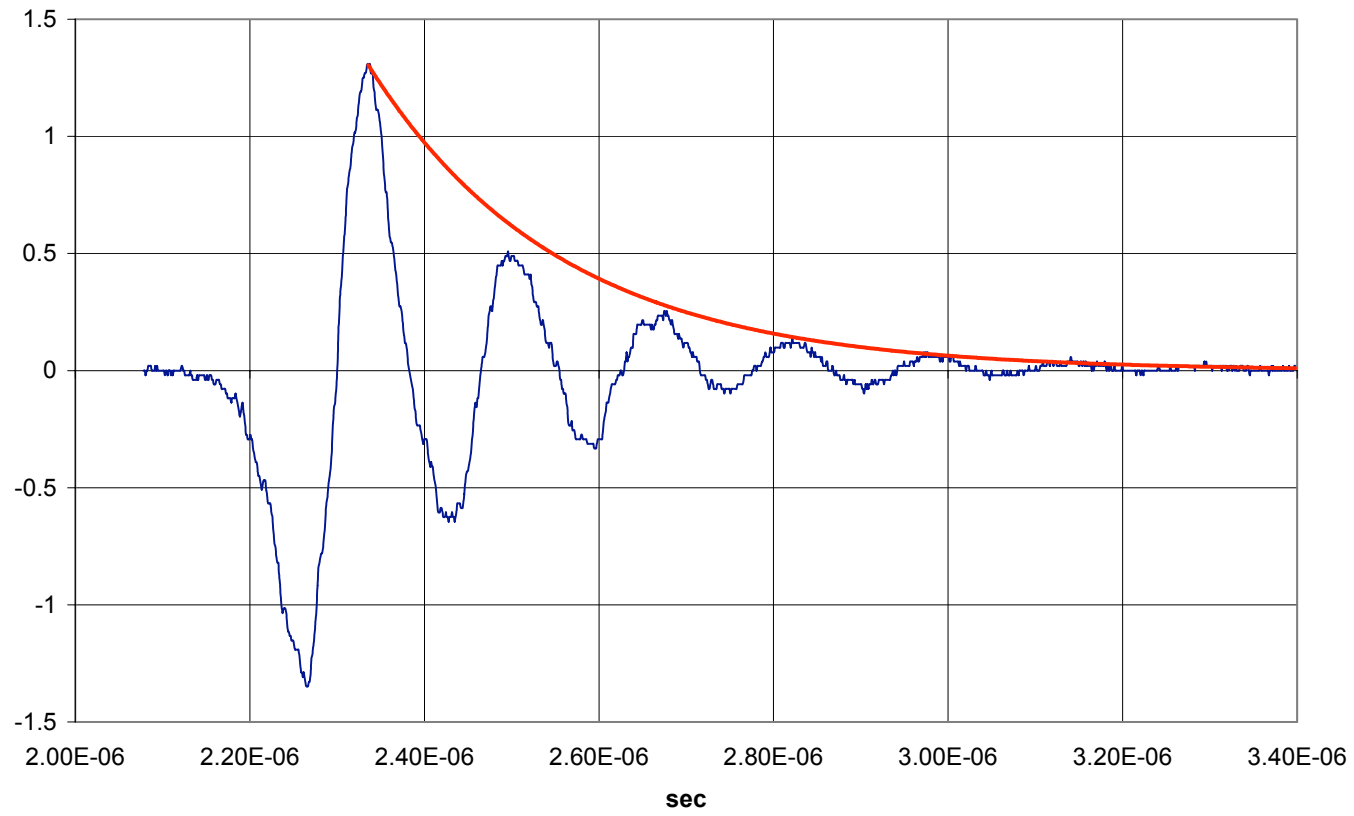

Figure 6.14 B-Dot \#3 signal for shot \# 3054 with a fit of the amplitude decay to an exponential (red curve). Note the expanded time scale relative to Figure 6.2. 
nonlinearities. The beam itself, modeled as a $\sim 2 k \Omega$ resistor, would contribute a damping rate of $1 / \tau_{b}$, with $\tau_{b} \approx 1.25 \mu \mathrm{sec}$. If we use $\tau_{0} \approx 0.25 \mu \mathrm{sec}$ for the decay constant in the "no beam" case, a perturbation calculation of the rate with the beam would predict a decay rate

$$
1 / \tau=1 / \tau_{b}+1 / \tau_{0}
$$

or $\tau \approx 0.21 \mu \mathrm{sec}$.

The center frequency of the oscillation is again about $6.1 \mathrm{MHz}$ as indicated by the FFT of B-Dot \#3 shown in Figure 6.15.

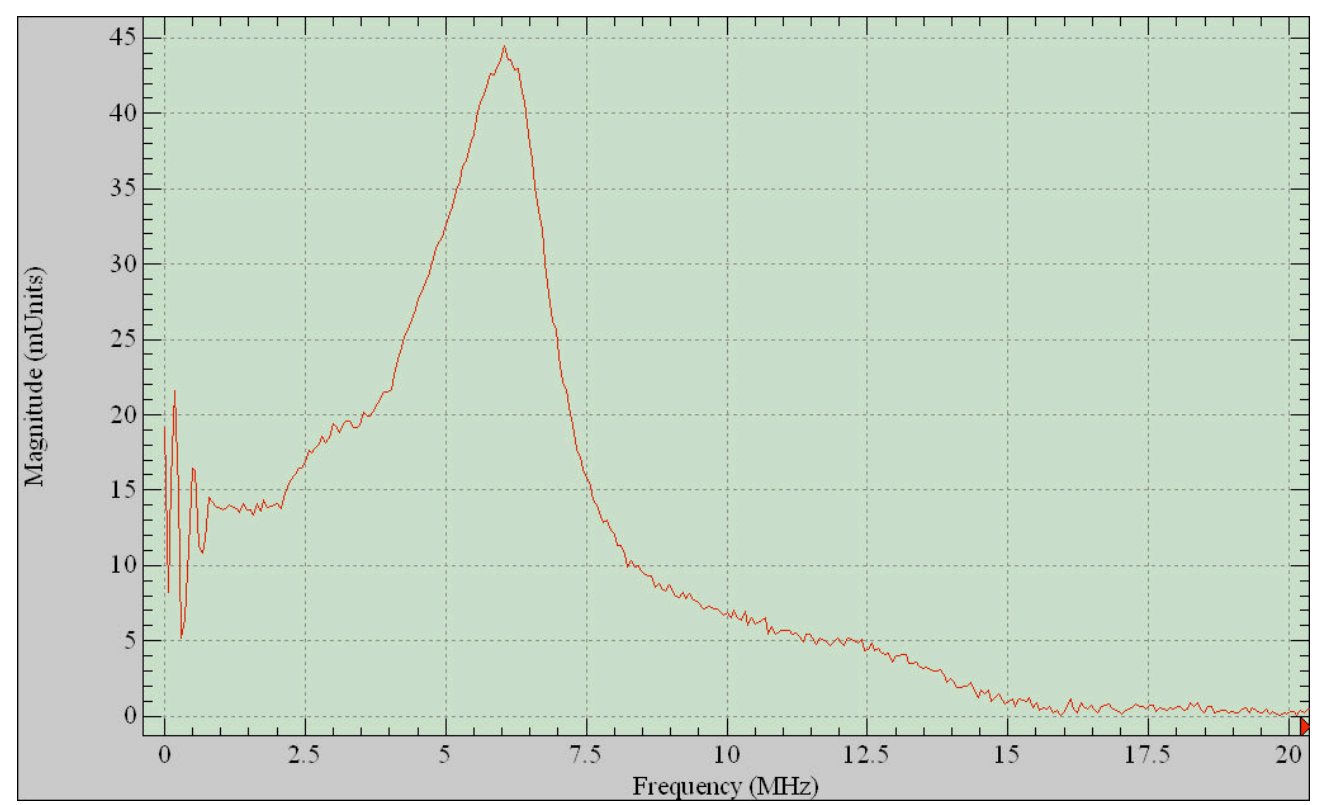

Figure 6.15 FFT of B-Dot \#3 for shot \# 3054. 


\section{Conclusion}

Although no criteria on the acceptable level of the $7.8 \mathrm{MHz}$ fluctuations in the flat top of the injector voltage waveform had been developed when the final design choices had to made, the ferrite damper has proven adequate in its enhancement of the damping rate. A summary of the exponential oscillation decay times with and without the ferrite damper for "no beam" and $\sim 1 \mathrm{kA}$ beam shots are summarized in Table 7.1.

\begin{tabular}{|lll|}
\hline & No ferrite damper & With ferrite damper \\
\hline No beam (cold cathode) & $0.8 \mu \mathrm{sec}$ & $0.25 \mu \mathrm{sec}$ \\
\hline With $\sim 1 \mathrm{kA}$ beam & $0.5 \mu \mathrm{sec}$ & $0.2 \mu \mathrm{sec}$ \\
\hline
\end{tabular}

Table 7.1 Summary of oscillation decay times comparing data before and after the installation of the ferrite damper.

The transient voltage stress on the ferrite insert during the rise time is modest at 3-4 $\mathrm{kV} / \mathrm{cm}(120 \mathrm{kV}$ peak over 13 " in a $20 \mathrm{~ns}$ pulse). The crowbar-induced oscillations in the ferrite voltage will be much larger. From the small scale circuit, the average stress may be approximately 4-5 times higher, which is still modest for a short pulse.

Additional analysis is warranted to assess the performance of the ferrite damper if the crowbar were to be used again. In particular, a major uncertainty will be the level of the higher frequency oscillations on the stalk current excited by the crowbar. 


\section{References}

1. "DARHT-II Beam Position Monitor B-dot Owner's Manual”, LANL document, September 12, 2002.

2. Nielsen, McCuistian, and Kirby, DARHT Injector Modification Design Review, August 7, 2002.

3. Henestroza, Briggs, and Yu, "Modeling Injector Oscillations in DARHT-2", informal LBNL/DARHT note, April 4, 2004.

4. Kang, "Preliminary Note on Modeling the DARHT2 Injector", January 3, 2003.

5. Henestroza, et al., "Injector Cavity Modes”, PAC 2003.

6. Briggs, et al., "Ferrite Inserts for Damping Injector RF Oscillations (I-Rev)", May 24, 2004 (original), Revised version July 6, 2005.

7. Reginato, Briggs, and Waldron, "Ferrite Inserts for Damping Injector RF Oscillations (II); Experimental Tests with a Scaled Circuit Model", October 6, 2004.

8. Injector Damping Interim Design Review, July 13, 2005, Committee Final Report (July 25, 2005).

9. Briggs and Fawley, "Campaign to Minimize the Transverse Impedance of the DARHT-2 Induction Linac Cells", DARHT Tech. Note 424, LBNL-56796 (REV 1), March 15, 2005. 